\title{
ELECTRON TRANSFER AT SENSITIZED SEMICONDUCTOR ELECTRODES
}

Mark Thomas Spitler

(Ph. D. thesis)

March 1977

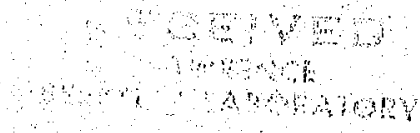

$413 \%$

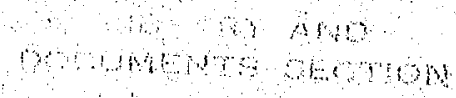

Prepared for the U. S. Energy Research and

Development Administration under Contract W-7405-ENG-48

\section{For Reference}

Not to be taken from this room

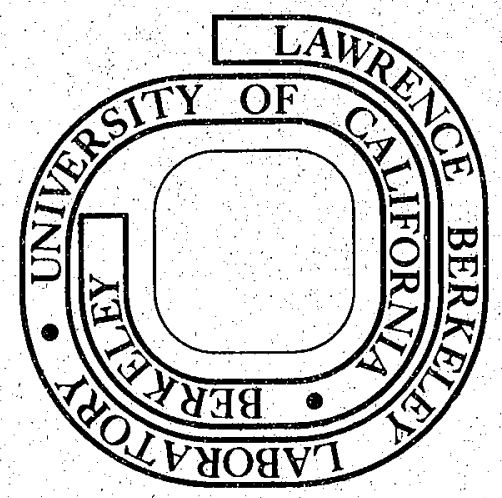


LEGAL NOTICE

This report was prepared as an account of work sponsored by the United States Government. Neither the United States nor the United States Energy Research and Development Administration, nor any of their employees, nor any of their contractors, subcontractors, or their employees, makes any warranty, express or implied, or assumes any legal liability or responsibility for the accuracy, completeness or usefulness of any information, apparatus, product or process disclosed, or represents that its use would not infringe privately owned rights. 


\section{$00+04710004$}

\section{ELECTRON TRANSFER AT SENSITIZED SEMICONDUCTOR ELECTRODES}

\section{CONTENTS}

Abstract

Acknowl edgements

I. Introduction

II. Adsorption of Sensitizing Dyes on Single Crystal Substrates

A. Introduction 3

B. Materials 5

C. General Experimental 10

D. Results of Adsorption Studies 14

1. Rose bengal on ZnS single crystals 16

2. Crystal violet on ZnS single crystals 16

3. Rose bengal on $\mathrm{Li}$ doped ZnO single crystals 19

4. Rose bengal and crystal violet on $\mathrm{ZnO}$ powder 21

5. Rhodamine B on undoped ZnO single crystals 25

E: Analysis 30

F. Discussion 40

III. Photooxidation of Rhodamine B at Zn0 Electrodes 48

A. Introduction 48

B. Background 48

1. Dye semiconductor energetics 48

(a) electronic structure of semiconductors 48

(b) energy relationship between dye and semiconductor 54

2. Semiconductor electrochemistry 56

3. Experimental apparatus for photoelectrochemical measurements 
C. Rhodamine B Sensitization of Zn0 66

1. Introduction 66

2. Materials 66

3. Results 67

(a) Comparisons with adsorption data 67

(i) action spectra 67

(ii) time decay $\quad 69$

(iii) photocurrent vs. solution concentration 72

(b) Time decay of photocurrent $\quad 77$

(i) introduction $\quad 77$

(ii) results $\quad 78$

(iii) derivation of a kinetic model 85

(iv) discussion 91

(c) Photoreduction of rhodamine B at the ZnO electrode 93

(i) introduction 93

(ii) experimental 94

(iij) discussion $\quad 94$

IV. Electron Transfer at Rose Bengal Sensitized $\mathrm{TiO}_{2}$ Electrodes 99

A. Introduction 99

B. Experimental 100

C. Results 102

1. Adsorption measurements 102

2. Dye photooxidation 104

3. Temperature dependence 115

4. Sensitized photoreduction 118

D. Discussion 122

1. Adsorption isotherms 122 
2. Excitation transfer 126

3. Dye-semiconductor energetics 129

4. Solution photochemistry 134

Appendix I. Expansion of Exponential Decay 138

Appendix II. Conductance of Semiconductors 139

$\begin{array}{ll}\text { References } & 140\end{array}$ 
' 


\section{7}

\section{ACKNOWLEDGEMENTS}

I wish to thank Professor Calvin for his support and advice during the completion of this work; his discussions were crucial to my scientific education. I also must acknowledge the very patient and probably very tired ears of Dr. Melvin Klein without which I would not have learned half so much. Professor Dan Haneman of U.N.S.W., Australia, contributed much towards the success of my initial efforts in this field.

To my wife Susan I owe a special debt; her unfailing support and confidence in me have sustained me during the more trying periods of my graduate career. To no less a degree I am in debt to my parents' belief in the value of my education; from the unswerving disinterest of Walter Radowiec and Gordon Richardson, I have received invaluable lessons in perspective. From Joe Smith I have learned much more - how to make beer.

I want to thank Terje Skotheim, Alan Robertson, Stanley Sorscher, Dr. Kevin McGregor, and Dr. Arthur Frank for their friendship and their entertaining, if not helpful, discussions.

Finally, I must express my appreciation for the cooperation and labors of Gloria Goldberg and Kathy Ward in the preparation of this manuscript. (And I mustn't forget Herman Bell who has kept me company on lonely nights in the lab).

This research was supported by the Division of Physical Research of the U.S. Energy Research and Development Administration. 


\section{INTRODUCTION}

Sensitization of a solid is a general term, ${ }^{1}$ one that can be applied to systems ${ }^{2-5}$ much different from the specific case which will be discussed in detail in the following pages. The basic elements of the process are, however, always the same. ${ }^{1,6}$ A sensitizing agent which can undergo an electronic transition must be in physical contact with a solid; the excitation of such an adsorbed species results in the induction of an electrical effect in the solid.

This electrical effect can be induced through either of two mechanisms: energy transfer between the adsorbed compound and solid or electron transfer between the two. Experimental observation of both of these processes has been claimed, ${ }^{7,8}$ al though it is evident that the predominance of one or the other will depend on the particular system under study. In either case, the efficiency of a sensitized mechanism is much smaller than that of intrinsic absorption of a photon by the solid itself, but it is initiated at a different, perhaps more desirable, wavelength.

The extension of the sensitivity of a solid to spectral regions beyond its intrinsic absorption has many practical applications of which the more significant utilize sensitizers that absorb visible radiation. With this use of dyes as sensitizers, spectral sensitization in the visible can be seen as the basic process behind modern photographic and duplicative techniques. More recently it has attracted attention as a possible means of solar energy conversion. ${ }^{9}$

Stemming from these interests, research in the field of dye sensitization of semiconductors has been long lived and has subjected this system to a great variety of investigative techniques. ${ }^{5,10,11}$ One of the more versatile methods for exploration of this phenomenon can be found in an electrochemical technique introduced by Tributsch and Gerischer. 10,12-14 
This approach to the study of sensitization utilizes the semiconductor as an anode or cathode in an electrochemical cell. The dyestuff is either deposited on its surface or dissolved in the electrolyte from which it adsorbs on the semiconductor. Work with this system has demonstrated that for sensitization of semiconductors in this configuration, the induced electrical effects are attributable to electron transfer to, or from, the excited adsorbed dye molecule. ${ }^{15}$

Thus, when the electrode surface is illuminated, the electron transfer between the solid and the excited dye results in current flow through the electrochemical circuit. The ability of a dyestuff to sensitize can then be simply and quantitatively measured.

This technique was selected and used for these studies of the photooxidation of water soluble dyes adsorbed on the surface of single crystal semiconductor electrodes. There were two major goals. One was to define the condition of the adsorbed dye. This required knowledge of its absorbance spectrum, its heat of adsorption, and its surface concentration, and a determination of the degree to which excitation is transferred between adsorbed dye molecules. The other goal was to establish quantitatively the energetic relationships between the adsorbed dye and the underlying semiconductor. It is hoped that this work represents some progress towards these ends. 


\section{ADSORPTION OF SENSITIZING DYES ON SINGLE CRYSTAL SUBSTRATES}

\section{A. Introduction}

The sensitization process described above is a surface reaction. Only those dye molecules adsorbed on the surface of the appropriate semiconductor can inject charge carriers into its electronic structure; though the photochemistry of sensitizing dyes in solution is well known, 16 the reactive species in the sensitization process is located in an unknown environment on the surface of the solid.

In order to acquire a complete understanding of the photooxidation or photoreduction reaction, this environment and its effect upon the adsorbed dye must be ascertained. Such a definition of the condition of the dye in its adsorbed state is not possible without answers to the following questions. How tightly is the dye bound to the surface? What forces are responsible for the adsorption of the dye to the surface? What is the adsorption orientation? What effect do these forces have upon the physical and electronic structure of the dye?

It is the purpose of the experimentation of this section to provide this information in the case of sensitization of single crystal substrates. This is certainly not the first piece of research into the surface chemistry of sensitizing dyes as the literature of photographic science is replete with studies of dyes adsorbed on silver halide powders. 1 However, use of a photoelectrochemical arrangement to monitor sensitization at specific crystallographic faces of solids necessitates concomitant studies of the dye adsorption on these we11 defined surfaces.

The techniques for obtaining this information about the effect of adsorption upon dye photochemistry have, of course, been established for powders as substrates. 1 For example, adsorption isotherms can be 
measured, often at several temperatures. The chemical composition of the substrate can be modified through doping. The solvent for these adsorption systems may also be changed, and reflectance spectra of these dyed powders can be measured at different surface concentrations of the adsorbate.

For single crystalline substrates, however, these methods become more difficult to apply. The small surface area of these crystals (>1 $\mathrm{cm}^{2}$ ) will adsorb correspondingly minute amounts of the dye on the order of $10^{14} / \mathrm{cm}^{2}$. An estimate for the optical density of this dye concentration on a surface would be .01 . Measurement of an adsorption isotherm is no longer an easy task. Adsorption spectra become harder to determine.

of these two types of measurements, the electronic excitation spectrum can reveal the most information about the interaction between the adsorbed dye and substrate. Charge transfer complexes, aggregate formation and the dielectric of the environment a11 are manifest in the electronic absorption of a molecule. In addition, if the transition moment of a molecular excitation is conserved upon adsorption from solution, its surface concentration, and thereby its adsorption isotherm, can be established. ${ }^{17}$ Finally, because it is this excited electron that is involved in the photooxidation of the dye, this absorbance spectrum can be compared to the photocurrent action spectrum determined through electrochemical studies. Thus, it appears that much usable information about the adsorbed species can be extracted from the absorbance spectrum of the dye on the surface of a crystal. Consequently, the effort was made to establish an experimental system that would enable measurement of the absorption spectra of dyes adsorbed on substrates at surface concentrations of less than $10^{14} / \mathrm{cm}^{2}$. 
At the time these experiments were begun, most electrochemical studies of sensitization had utilized $\mathrm{ZnO}$ as the semiconductor electrode. $12,13,14,18,19$ of al1 the wide band gap semiconductors, it had been the most extensively researched and consequently could be seen as a well defined component of a photoelectrochemical system. Thus, samples of this crystal were sought as suitable substrates for adsorption studies. However, specimens of this crystal were not immediately available. Consequently, easily obtainable ZnS crystals which had the same crystal structure as $\mathrm{Zn} 0$ were employed to explore the experimental techniques for the adsorption studies.

Of the dyes that produced a photo-effect at the $\mathrm{ZnO}$ electrode, the two most efficient sensitizers were rose bengal and rhodamine B. $6,13,14$ Therefore these dyes were selected for use as sensitizers because the results could be compared with easily obtainable electrochemical data. However, the photocurrent action spectra of these dyes revealed that they most likely adsorbed as monomers. ${ }^{20}$ In order to work with a system where aggregation occurred on the surface, crystal violet was also included for use in these single crystal studies.

\section{B. Materials}

Crystal violet (MW 425.97) is a water soluble, triphenylmethane dye whose structure is shown in figure 1. It is cationic and planar forming dimers at relatively low solution concentrations. The absorption spectra of the dye as a monomer and dimer are also shown in figure 1. At the monomer maximum in ethanol, the extinction coefficient is $\times 10^{5}$. The dimer absorption is blue shifted from the monomer maximum. 

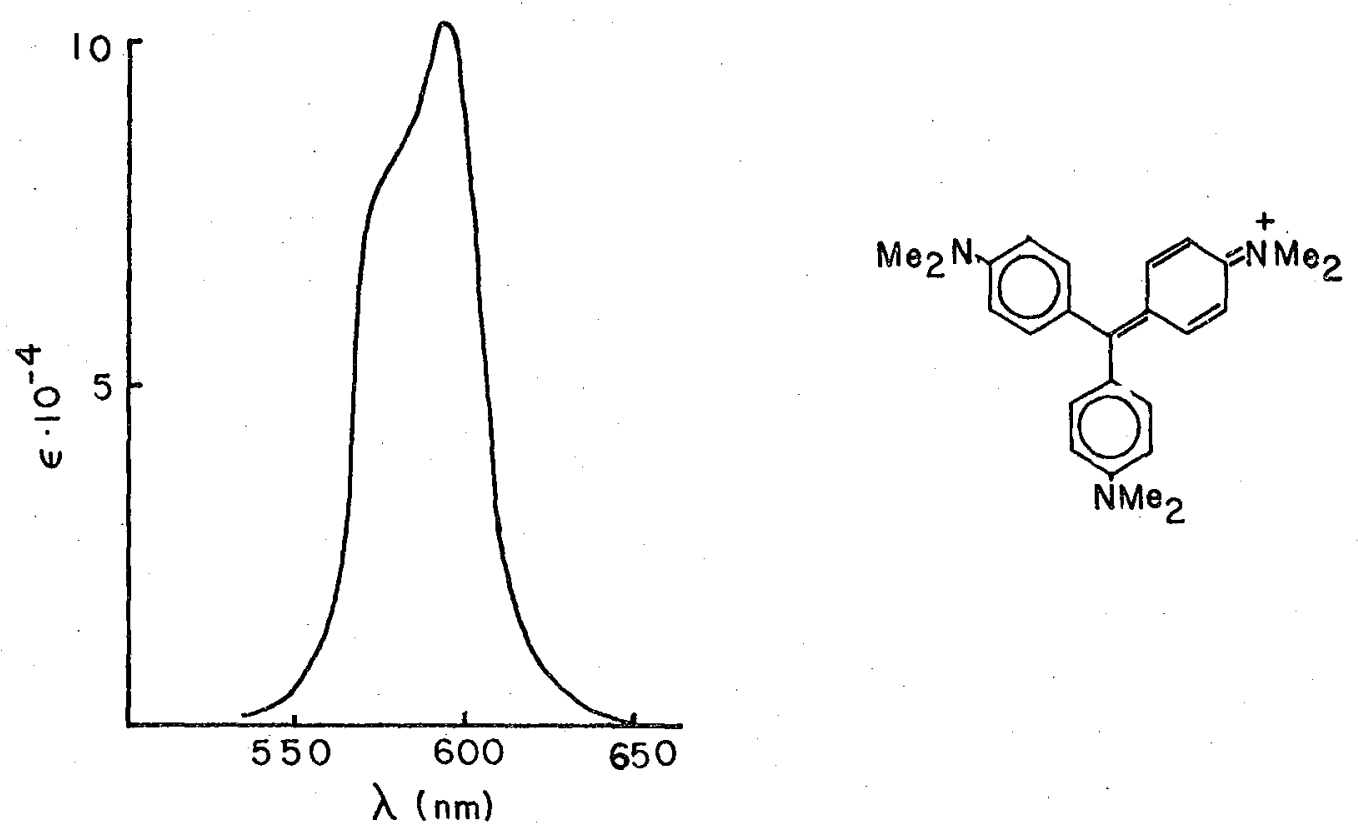

Figure 1. Structure and absorption spectrum in ethanol of crystal violet.
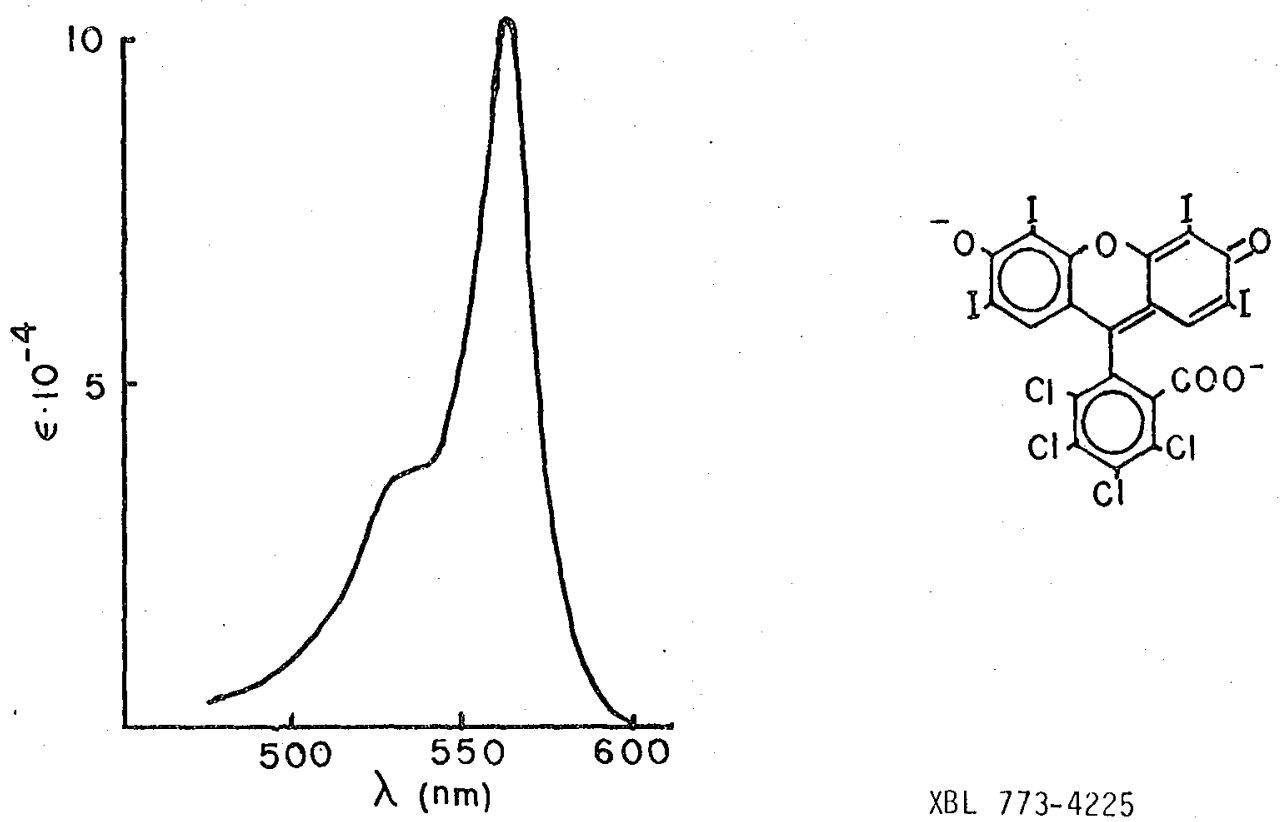

Figure 2. Structure and absorption spectrum in ethanol of rose bengal. 
Rose bengal (MW 1019.52) is the common name for tetraiodo, tetrachlorofluorscein and retains the basic spectral features of fluorescein albeit red shifted to about $550 \mathrm{~nm}$ (figure 2). The dimer has an absorption maximum at an energy equal to the shoulder of the monomer. The substituents on the phenyl ring constrain its orientation perpendicular to the remainder of the molecule so that dimerization is inhibited until fairly high concentrations have been reached. Below pH 5 the dye precipitates from aqueous solutions with the protonation of its acidic functions.

The material was purchased from Allied Chemicals and recrystallized from ethanol. After this purification the extinction coefficient of the monomer peak in ethanol was determined to be $1.05 \times 10^{5}$ which is the value reported in the literature.

The structure of rhodamine $B$ is shown in figure 3 . Its spectral characteristics are similar to rose bengal, but the high energy shoulder on the monomer is not as pronounced. Steric effects orient the phenyl group perpendicular to the plane of the chromophore for this molecule also. Dimerization results in an increased absorption at shorter wavelengths. At the monomer maximum in ethanol, the extinction coefficient is $1.10 \times 10^{5}$. This dye was also purchased from Allied Chemicals and purified through recrystallization in ethanol.

The net charge on the dye will depend on the pK of the carboxyl function; the molecule will be either cationic or neutral. To determine the region of charge neutrality for this dye, gel electrophoresis was used. An LKB 7900 Uniphor Column Electrophoresis system was employed for this determination.

The dye was spread eventy over the entire $\mathrm{pH}$ range of a supporting 


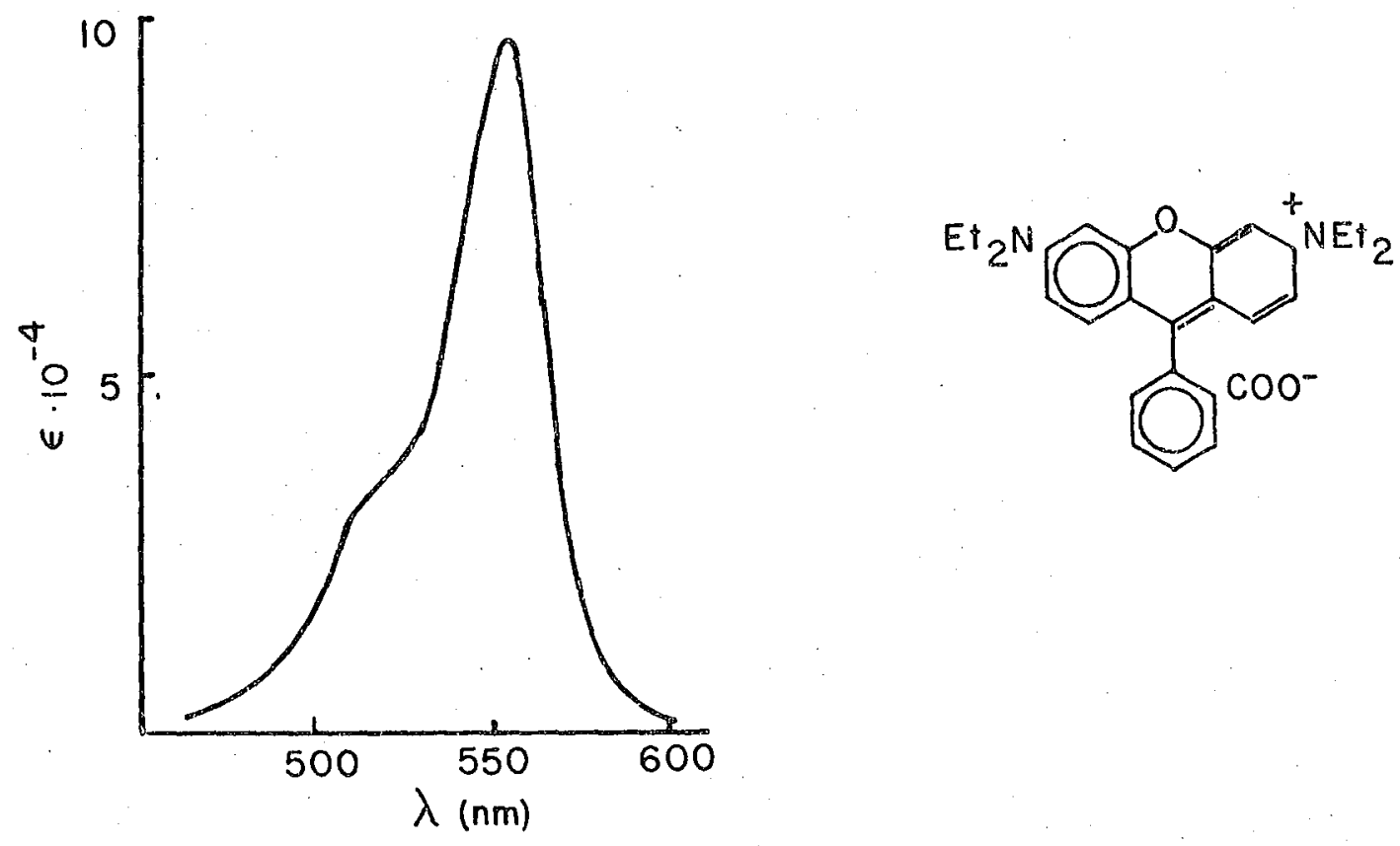

Figure 3. Structure and absorption spectrum in water of rhodamine B.

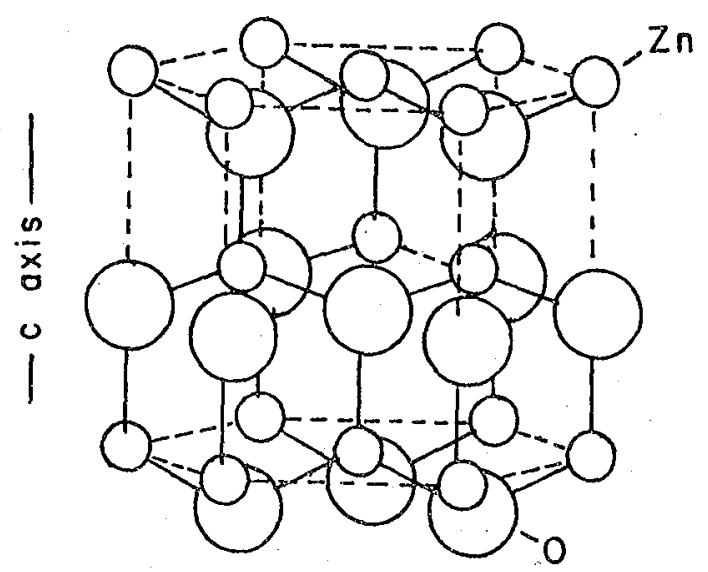

Figure 4. The $\mathrm{ZnO}$ and $\mathrm{ZnS}$ single crystals used in these studies crystallized in the hexagonal wurtzite structure pictured above. The (0001) face consists purely of Zn while (0001) face is 0 or $S$, The lattice constants for Zno are $a=3.25 \AA, c=5.12 \AA$ and for $\mathrm{ZnS}, \mathrm{a}-3.81 \AA, \mathrm{c}=6.23 \mathrm{R}$. 
gel. After 48 hours in this set-up, all the dye at low pH had moved to a higher $\mathrm{pH}$. There was a distinct break between colorless gel and dyed gel at a pH of 4.85. Higher than this $\mathrm{pH}$ there appeared to be no movement of the dye indicating that above $\mathrm{pH} 4.85$ the dye is electroneutra1.

This result is not in accord with a reported 21 pK of 9 for this carboxyl group. However, it is in agreement with other data in the literature which show a change in the fluorescence and absorbance of rhodamine solutions near $\mathrm{pH} 5.15$

Zinc oxide is a semiconductor with a band gap of $3.2 \mathrm{eV}$. It is therefore transparent to visible light and usually crystallizes as hexagonal prismatic needles with a wurtzite lattice structure. 20,21 $\mathrm{ZnO}$ is a noncentrosymmetric crystal (figure 4), so that a crystal cleaved perpendicular to the longitudinal axis of a hexagonal prism will have two different faces exposed, one $\mathrm{Zn}(0001)$, the other 0 $(0001)$.

Crystals grown through vapor deposition without intentional dopants are usually $n$ type as a result of interstitial zinc atoms within the lattice. 24 These zinc atoms function as donors since they are essentially completely ionized at room temperature.

The ZnO used in these adsorption studies was obtained from two sources. A plentiful supply was received as a gift from Dr. Gunther Heiland of the Technische Hochschule, Aachen. These came in the form of long $(2-3 \mathrm{~cm})$ hexagonal prisms grown through vapor deposition. A large $2 \mathrm{~cm} \times 1.5 \mathrm{~cm} \times 1 \mathrm{~mm}$ plate crystal of $\mathrm{ZnO}$ was purchased from the Lambda-Airtron Corporation of Morris Plains, New Jersey. This 
specimen exposed large areas of the $\mathrm{Zn}$ and 0 surfaces. However, the crystal was hydrothermally grown in a $\mathrm{LiOH}$ solution which introduced Li into the lattice and lowered its conductivity.

Zinc sulfide is also a wide band gap semiconductor $(3.6 \mathrm{eV})$ that crystallizes in two lattice structures. One is in the diamond form; the other is in the wurtzite structure (figure 4) which was the habit of the crystal used in these experiments. Commercial samples of $\mathrm{ZnS}$ are, in general, insulating, and the material purchased was left undoped since no electrochemical studies were planned for this crystal. A $10 \mathrm{~g}$ boule of single crystalline $\mathrm{ZnS}, 2 \mathrm{~cm}$ in diameter, was purchased from Eagle-Picher Industries of Miami, Oklahoma.

\section{General Experimental}

The investigation of the adsorption of sensitizing dyes on single cyrstalline substrates required that the visible absorption of dyes on semiconductor surfaces be known. Even if the surface is covered with a complete monolayer of the dye, the maximum absorbance of that dye layer will only be ca .01. Although this optical density is small, it can be accurately measured if the substrate is fashioned into a thin plate crystal and placed in the beam of one of the Cary spectrophotometers available in the laboratory.

However, at sub-monolayer surface concentrations of the dye this accuracy will suffer. Consequently, some means had to be found for determining the optical density of adsorbed dye molecules at very low surface concentrations.

The first method which presented itself utilized the property of total internal reflection of light within a solid dielectric medium. This technique has been explored and explained in detail in an excellent 
book by N. J. Harrick. If the geometry of the optics and of the crystal are correct (see figure 5a), visible light entering the semiconductor will be totally reflected at each gas-solid boundary encountered. Thus, before the light path leaves the solid it can be made to cross through a semiconductor-dye interface enough times to obtain an accurate measure of the absorbance of the adsorbed dye. This is a very elegant physical technique, but it demands extreme precision in its optical and geometrical arrangements. A simpler and easier arrangement was sought and found.

Instead of using one crystal for absorbance measurements, it was determined that several crystals could be employed instead of one if they were aligned as plate crystals perpendicular to the beam of a spectrophotometer. Each additional semiconductor surface contributed an additional absorbance of .01 with as many as four crystal plates employed in this arrangement. Dye could also be adsorbed on both sides of the crystal.

Using this method it was possible to obtain absorbance spectra of adsorbed dye layers as a function of the solution concentration with which the surface dye is in equilibrium.

To align the crystal surfaces perpendicular to the light beam, a plexiglass optical cel1 was fabricated for use in a Cary 118 spectrophotometer with the capability of holding five crystals. The design of this device is seen in figure $5 b$. The crystals were mounted on thin plexiglass rectangles over a $3 \mathrm{~mm} \times 1 \mathrm{~mm}$ aperture which permitted passage of the light beam.

The highest measured optical density approached .08 for four surfaces. The smallest absorbance was limited by impurity absorption 


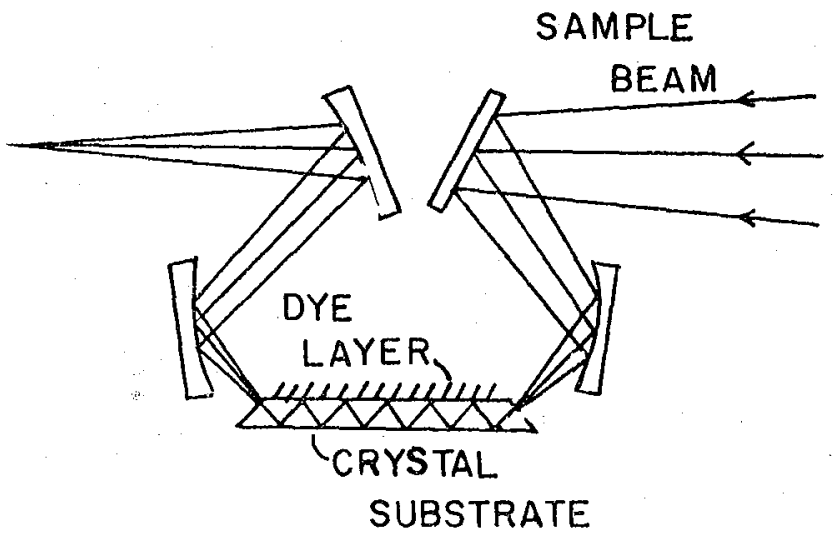

Figure 5. This diagram shows the geometry and optics required for use of total internal reflection to measure the absorbance of dye adsorbed on the surface of single crystals. Below is the design of a plexiglass optical cell that was used in these absorbance measurements. The crystal could be mounted on plexiglass slips which were inserted into the holder. Up to five crystals with ten dye layers could be inserted in the spectrophotometer beam at one time.
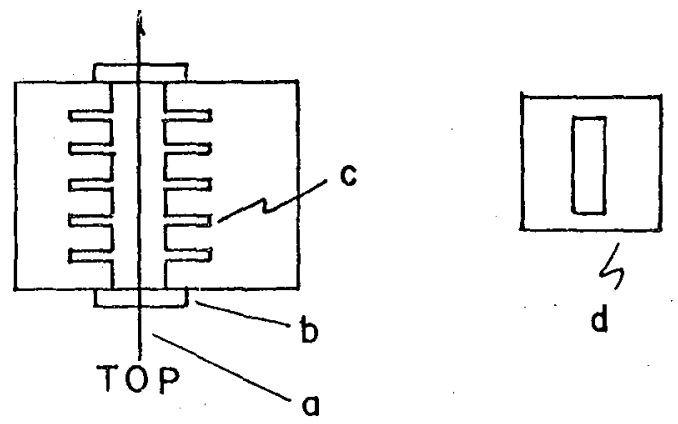

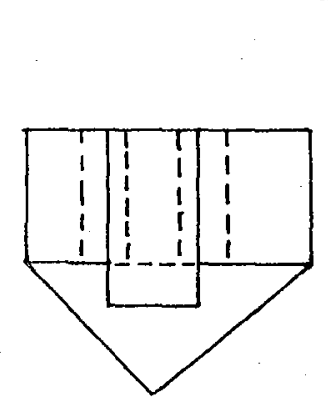

FRONT

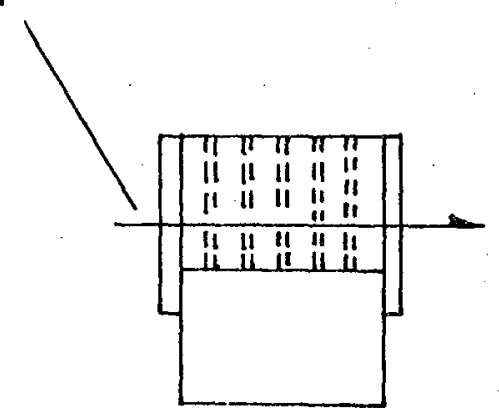

SIDE

(a) light path. (b) quartz window.

(c) mounting slots. (d) crystal mount. 
in the crystal and scattering of the light by the crystals. The impurity absorption of the crystals takes the form of a sloping baseline which decreases with increasing wavelength. The dye absorption is superimposed on this background absorption; an example is given in figure 2 for rose bengal on $Z$ nS. This presents very little problem because it can be subtracted easily. However, it does introduce uncertainties into the determination of the exact wavelength of an absorbance maximum. As will be demonstrated for the system rhodamine $B / Z n O$, this sloping baseline can be eliminated to permit accurate observation of spectral shifts as a function of surface concentration of the dye.

Only one problem presented itself when using this technique. The crystals used had to be large enough to handle easily so that an optical surface could be prepared on both faces through which the light passed. A minimum workable crystal surface was found to be an area $4 \mathrm{~mm}$ square. Finding Zno single crystals of this size proved to be difficult and time consuming. As a consequence, during the time in which the $\mathrm{Zn} 0$ was being located, the $\mathrm{ZnS}$ was used to sound the technique for its efficacy.

The dye on the semiconductor surface is adsorbed from a solution of the dye. The crystal is immersed in this dye solution until equilibrium is reached between the dye in solution and the dye on the surface. This usually required $15-20$ minutes.

In order to preserve the partition of dye between surface and solution when the crystal is removed from the solution, the liquid on the surface of the crystal must be removed without any evaporation. This can be accomplished by washing the crystal immediately with a solvent in which the dye is not soluble. It was found that if this liquid were squirted out of a plastic squeeze bottle, there will be sufficient force in the 
stream to remove any liquid on the surface of the crystal.

Solubility is a relative term, and it may be that this small

surface concentration of dye, $10^{14} / \mathrm{cm}^{2}$, can be solvated and eluted by the wash solvent. The possibility can be checked very easily. An absorption spectrum of dye adsorbed on a crystal is recorded after a wash with thisnonpolar solvent. The crystals can be removed from the optical cell and washed once again followed by another absorbance measurement. If the adsorbed dye is insoluble in the washing agent, the absorbance spectra of the adsorbed dye will remain unchanged after repeated washings.

Equilibrium in the adsorption of the dye onto the semiconductor from solution rarely required more than 20 minutes. This was established through a variation of the immersion time for each dye, and each dye concentration, and for each semiconductor. As expected, concentrated dye solutions attained equilibrium within a couple minutes whereas the more dilute ones required a longer time period.

\section{Results of Adsorption Studies}

This technique was first employed with $\mathrm{ZnS}$ as the substrate and rose bengal and crystal violet as sensitizing dyes. Adsorption studies of these dyes on ZnS powders was attempted for comparison purposes, but acidic surface characteristics of the powder resulted in protonation and decoloration of the dye solutions. ${ }^{26}$ Consequently, the experiments were performed with ZnO powder. Finally, single crystal studies using Zn0 were made with rhodamine $B$ and rose bengal.

As a rule, before a set of crystals was utilized for an adsorption study, the component crystals were soaked inchloroform and methanol to remove any soluble surface impurities and then boiled in doubly distilled 


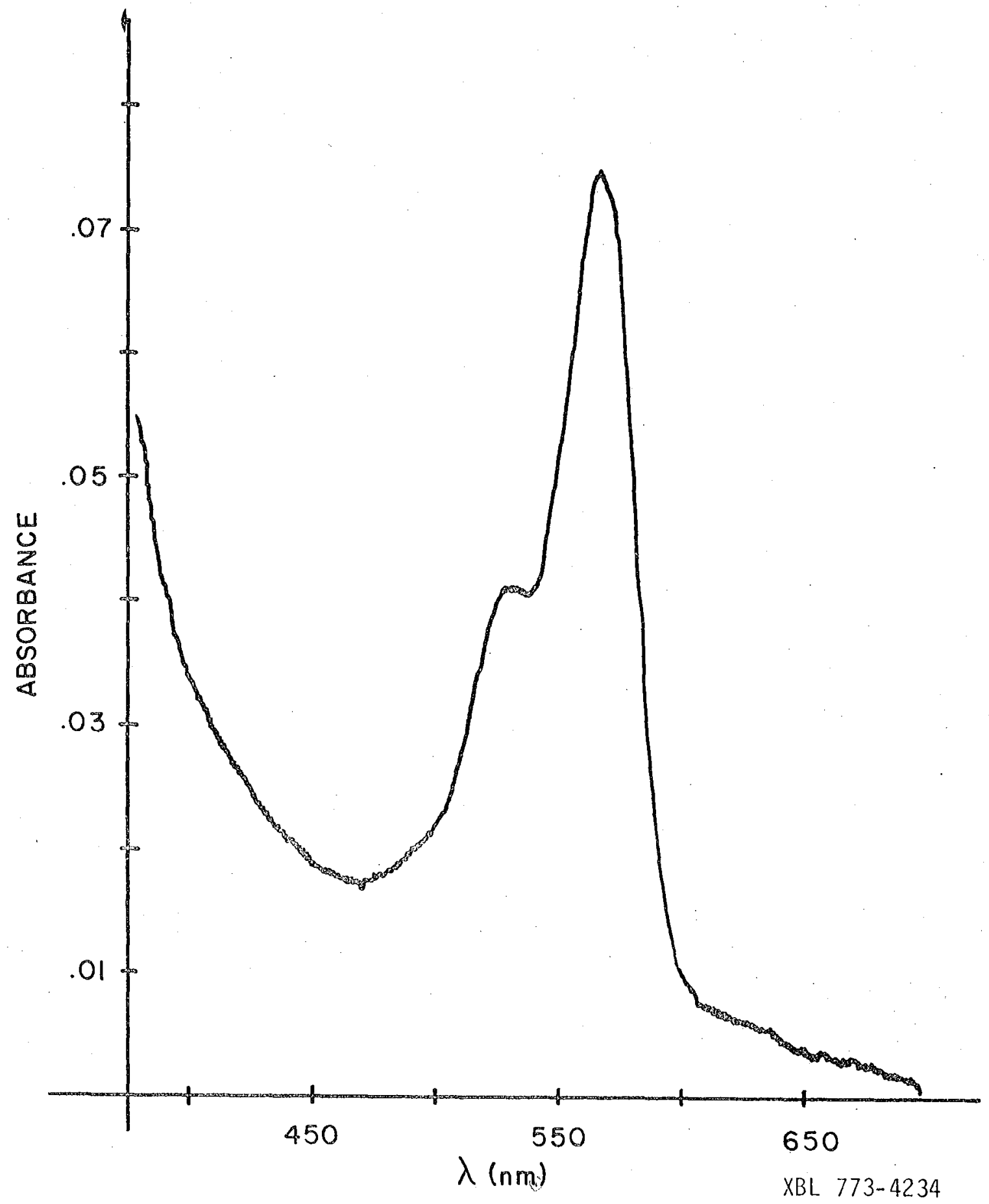

Figure 6. Rose Bengal adsorbed on $\mathrm{ZnS}$ single crystals from a solution that is $7.9 \times 10^{-5} \mathrm{M}$ in the dye. The absorbance represents the contribution of six surface layers of the dye superimposed upon the sloping absorption of the $\mathrm{ZnS}$. 
water for an hour. It was found that octane, nonane, and decane functioned very well as washing solvents for these dyes. Spectroquality solvents were used in a11 wash procedures.

1. Rose bengal on ZnS single crystals

Slices of the $\mathrm{ZnS}$ boule $1 \mathrm{~mm}$ thick were cut with a diamond saw perpendicular to the $c$ axis to expose the (0001) $\mathrm{Zn}$ and (0001) S faces. These wafers were cut into rectangular pieces $1.5 \mathrm{~cm} \times 0.3 \mathrm{~cm}$ to fit in the optical cell of figure 5b. The crystal surfaces were polished first with $9 \mu$ SiC grit and then with $1 \mu \mathrm{Al}_{2} \mathrm{O}_{3}$ powder. The crystals were not etched so that the adsorption experiments were carried out on polished surfaces.

Using both sides of only two crystals, the absorbance spectra of adsorbed dye in Figure 7a were obtained from crystals. If the absarbance of the maxima of these spectra are plotted as a function of the corresponding solution concentration, the graph of figure $7 \mathrm{~b}$ is obtained. As the dye in solution increases from $2 \times 10^{-5} \mathrm{M}$ to $5 \times 10^{-4} \mathrm{M}$, the surface absorbance remains constant. For purposes of comparison, the spectra of the dye in ethanolic solutions of varying concentrations are in figure 2.

The spectra of figure $7 \mathrm{a}$ resemble the absorption of the monomer in solution (figure 2). A red shift of $9 \mathrm{~nm}$ is evident.

2. Crystal violet on $\mathrm{ZnS}$ single crystals

The ZnS single crystals prepared above were also employed in the measurement of absorption spectra of crystal adsorbed from absolute ethanol. Solutions of the dye up to $6.0 \times 10^{-4} \mathrm{M}$ were used. The crystals were washed with cyclohexane. The absorbance spectra of the crystal violet on the $\mathrm{ZnS}$ and in solution are depicted in figures 8 and 1. 


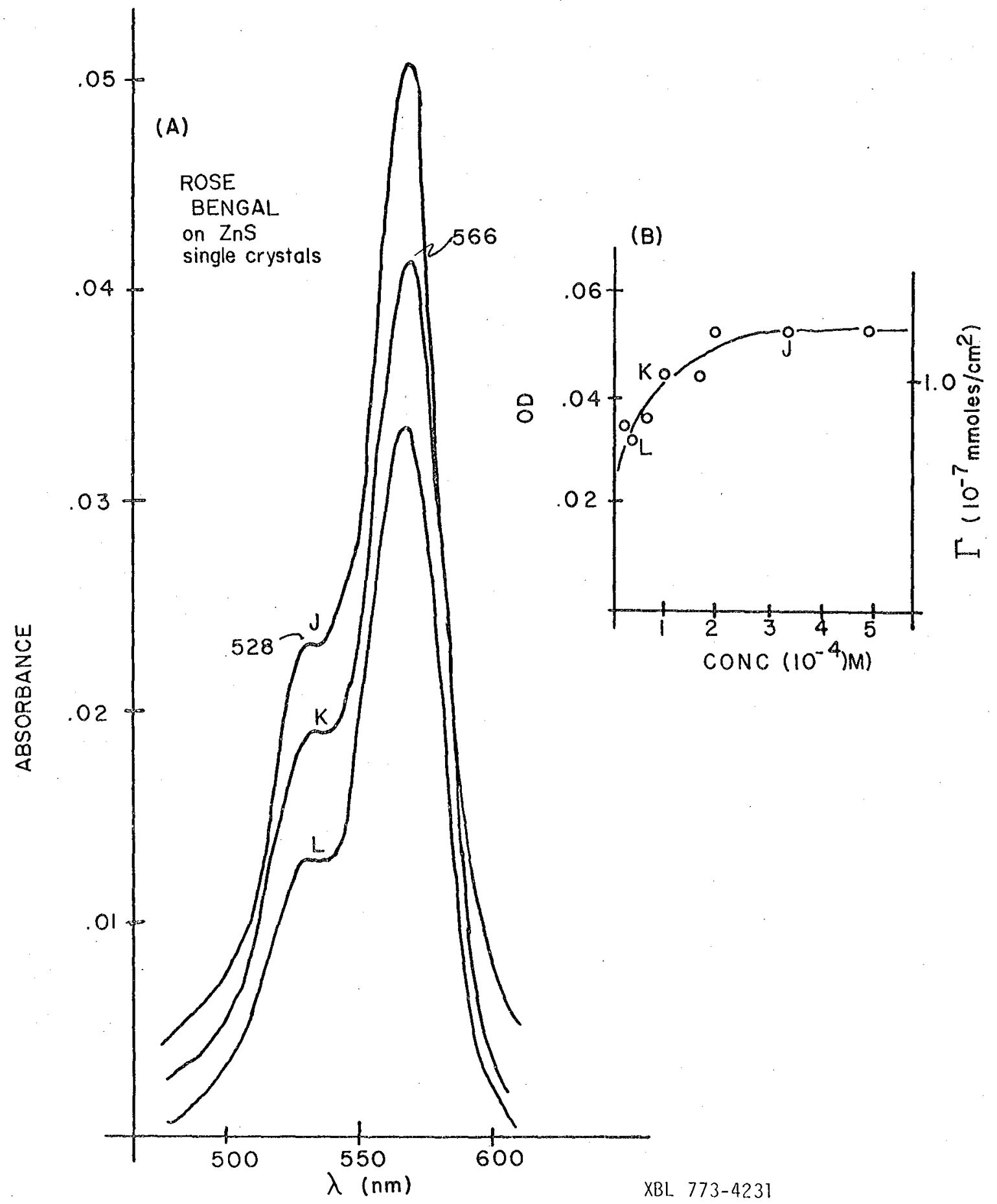

Figure 7. Absorbance spectra of rose bengal on ZnS single crystals as a function of surface concentration are shown in (a). In (b) is a plot of the absorbance of the adsorbed dye at $566 \mathrm{~nm}$ as a function of solution concentration. 


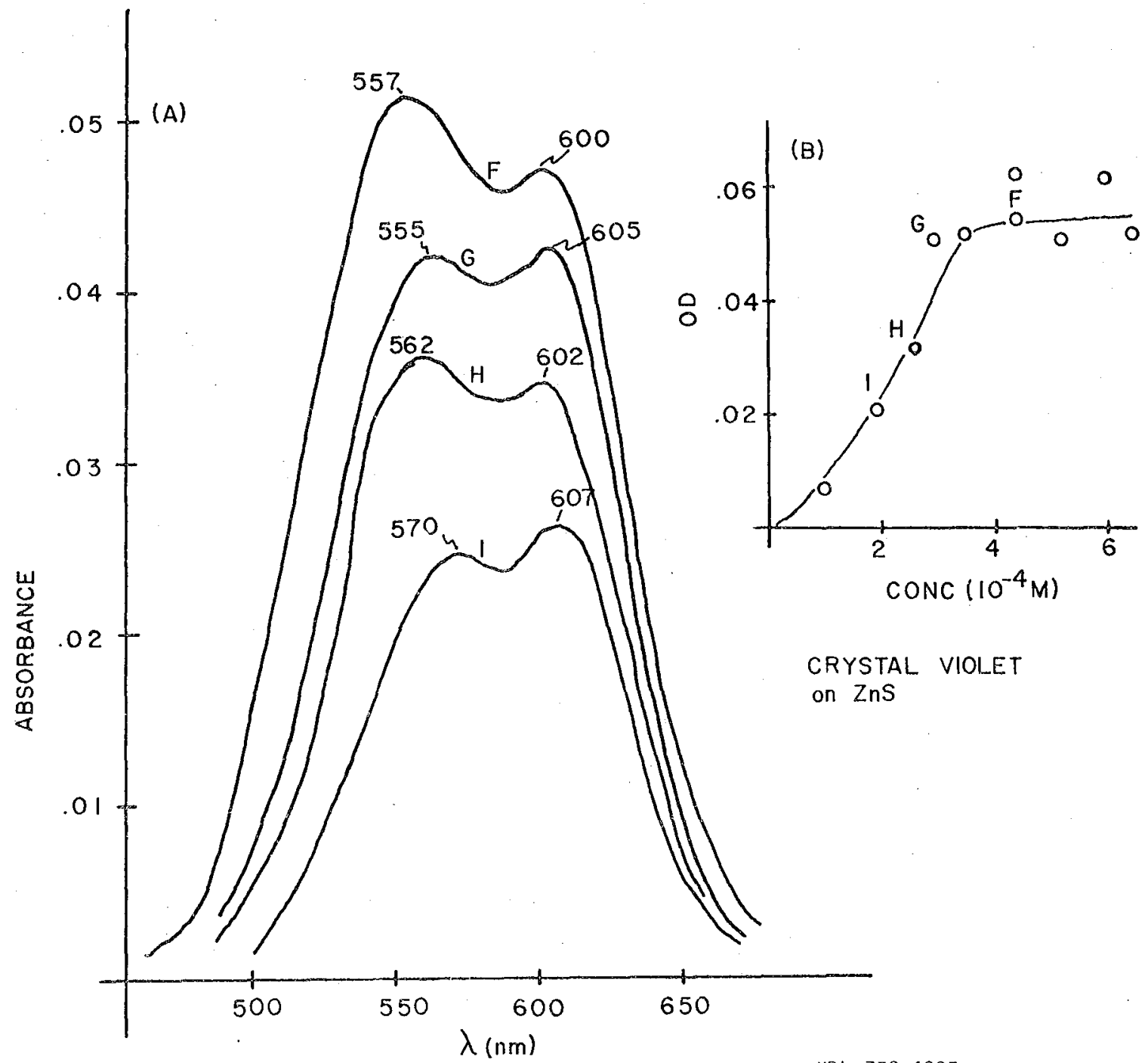

XBL 773-4227

Figure 8. Absorbance spectra of crystal violet on ZnS single crystals at several surface concentrations are shown in (a). In (b) the $0 D$ at the monomer maximum is plotted as a function of solution concentration. 
Comparison of these figures reveal that dimerization of crystal violet occurs on the surface. This tendency towards aggregation increases as the dye concentration on the surface becomes larger. The absorption maxima of both monomer and dimer on the surface are red shifted from the solution spectra by $\sim 15 \mathrm{~nm}$.

3. Rose bengal on Li-doped $\mathrm{Zn0}$

Only one crystal was used in these measurements. It was the plate crystal of Li doped ZnO obtained from Lambda-Airtron. The two exposed surfaces were the $\mathrm{Zn}(0001)$ and 0 (0001) faces so that the spectra reflected absorbance from dyes adsorbed on two chemically different surfaces. However, dyes adsorbed on these two surfaces gave identical absorption spectra so that this difference appeared to present no problem.

The crystal was polished to a $7 \mu$ finish like the $\mathrm{ZnS}$ specimens and used without etching. Absorption spectra of rose bengal from aqueous solutions of up to $6.0 \times 10^{-4} \mathrm{M}$ were recorded. The absorption spectra of the dye solutions corresponding to the spectra of figure 9 are shown in figure 2.

As with the adsorption of this dye on $\mathrm{ZnS}$, the surface species is predominately monomer in form. As the surface coverage increases, the absorbance of the high energy shoulder increases with respect to the main peak. This indicates an increasing dimer presence. These maxima also are at a longer wavelength than those in the solution spectra.

At this point it would be useful to compare the results found with single crystals with data obtained from adsorption of the dye on powders. Rose bengal and crystal violet were adsorbed onto $\mathrm{Zn0}$ 


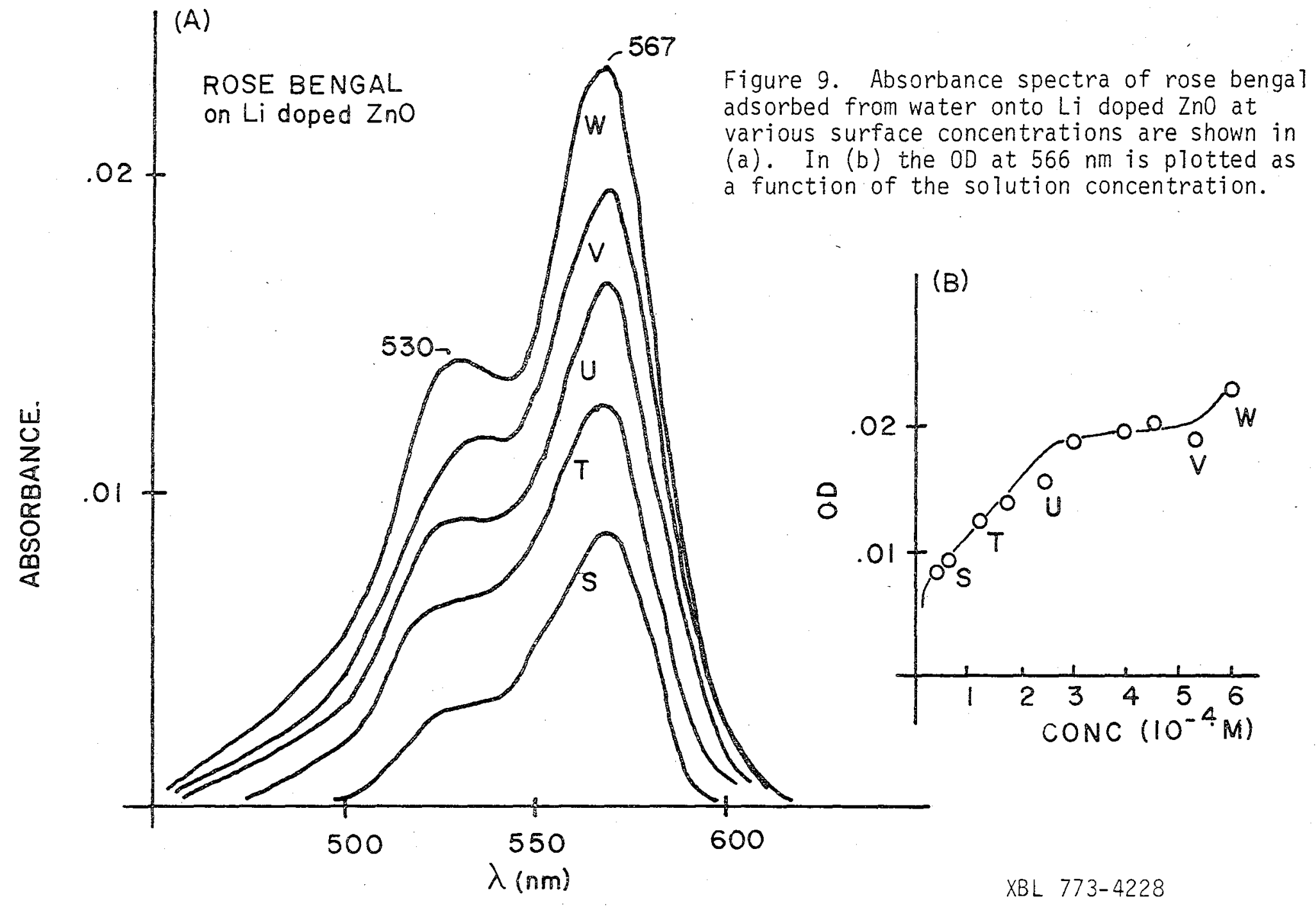




\section{6}

powders from ethanolic solutions. Adsorption isotherms for these two systems were established and reflectance spectra for rose bengal adsorbed onto $\mathrm{Zn} 0$ were recorded. Unfortunately, the crystal violet underwent a photochemical bleaching during the experimental procedure and reflectance spectra could not be measured for this dye.

4. Rose bengal and crystal violet on Zn0 powder

UTtrapure Zn0 powder (99.999\% pure) was obtained from Ventron. Electron microscopically the powder was found to be microcrystalline with prismatic and plate crystals ranging in size from $0.5 \mu$ to $2.0 \mu$. To establish a history for the powder, it was heated in air for 3 hours at $300^{\circ} \mathrm{C}$.

$200 \mathrm{mg}$ of the powder were deposited in a plastic sealable centrifuge tube with a $10 \mathrm{ml}$ aliquot of an ehtanolic solution of the dye. It was then shaken for a minimum of four hours which was the time required for the least concentrated solution to attain equilibrium. After this time the samples were centrifuged, and a $1 \mathrm{ml}$ sample was removed by pipette for a spectrophotometric determination of its concentration. The resultant adsorption isotherms are shown in figure 11. Each point represents the average of three samples.

The remaining solution in the centrifuge tube was removed and the powder was washed with cyclohexane. The cyclohexane was allowed to evaporate and the powder was saved. Reflectance spectra of the dye adsorbed onto the $\mathrm{ZnO}$ powder was saved. Reflectance spectra of the dye adsorbed onto the Zno powder were taken using a Cary 14 spectrophotometer with a Cary Model 1462 Scattered Transmission Accessory. With this apparatus, the photomultiplier tube could be moved to within a few millimeters of the sample cuvette so that it intercepted a large solid 


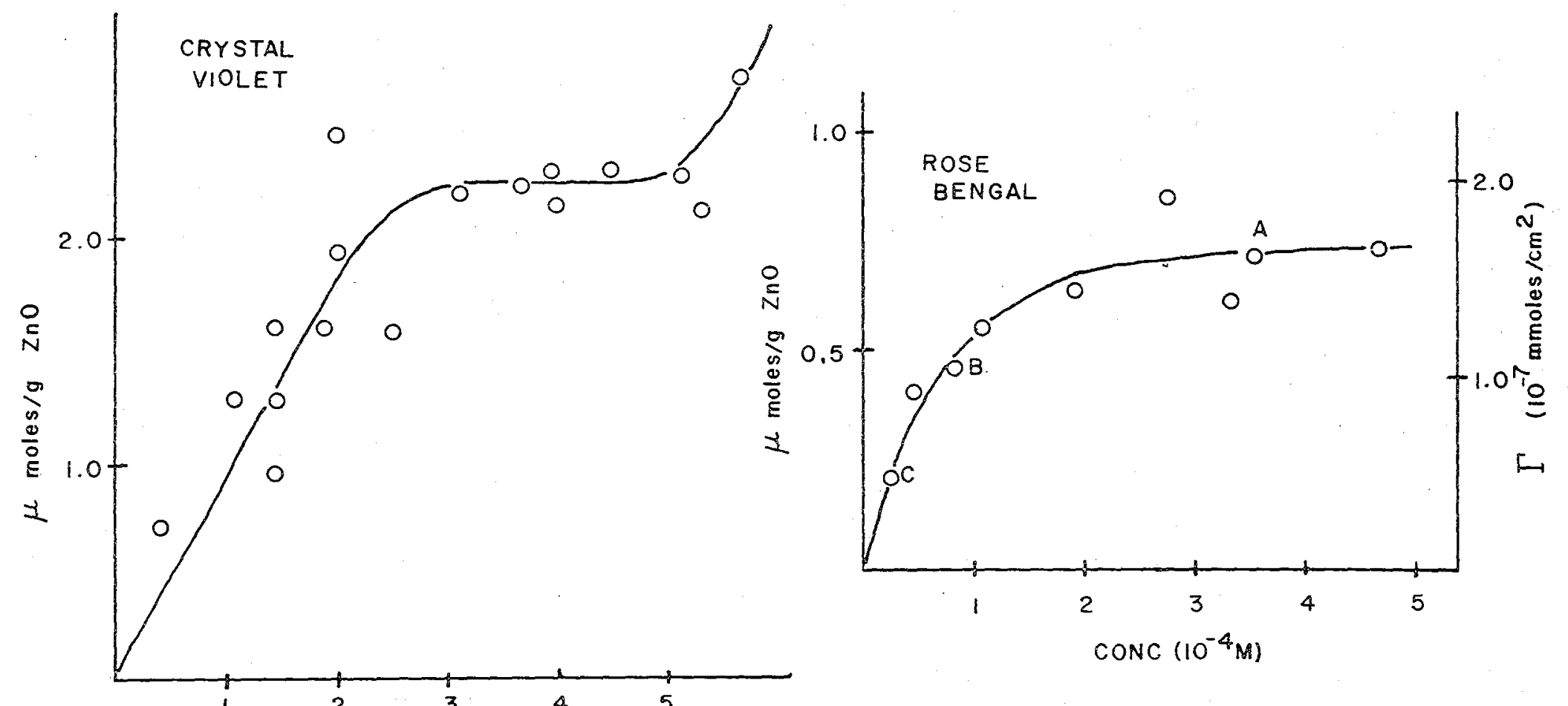

XBL $773-4230$

Figure 11. Adsorption isotherms of crystal violet and rose bengal on ZnO powder are shown here. 
angle of the light scattered by a sample.

The dry powder was suspended in an inert silicone oil (Dow 704 diffusion pump fluid) by a rapid stirring of a mixture of the solid and oil. Once this suspension was poured into the cuvette, the powder settled to the bottom within an hour. Only a couple minutes were required to record a spectrum so that no distortion was observed as a consequence of the settling process.

Reflectance spectra at several surface concentrations of the dye are depicted in figure 12. They appear very similar to the absorbance of the dye adsorbed on a single crystalline substrate. The monomer absorbance is present with a red shift from solution spectrum. The most significant difference between the absorbance of adsorbed dye molecules and those in solutions is the red shift of the absorption maximum of the dye when it is in the adsorbed state. For many dyes this red shift has been reported to increase with increasing surface concentration of the dye. Although there are changes evident in the crystal violet spectra of figure 8 , such a red shift is noì observed in the rose bengal spectra.

However, there is some uncertainty in these spectra owing to the sloping crystal baseline. To obtain accurate information on this red shift this baseline problem must be eliminated. This can be done by inserting crystals into the reference beam of the spectrophotometer so that the absorption of the impurities in the crystal will be compensated. This is a difficult task since the thickness of the crystal samples in the reference beam must be exactly equal to the thickness of the material in the sample beam in order to obtain complete cancellation. With future electrochemical studies in mind the dye-semiconductor 


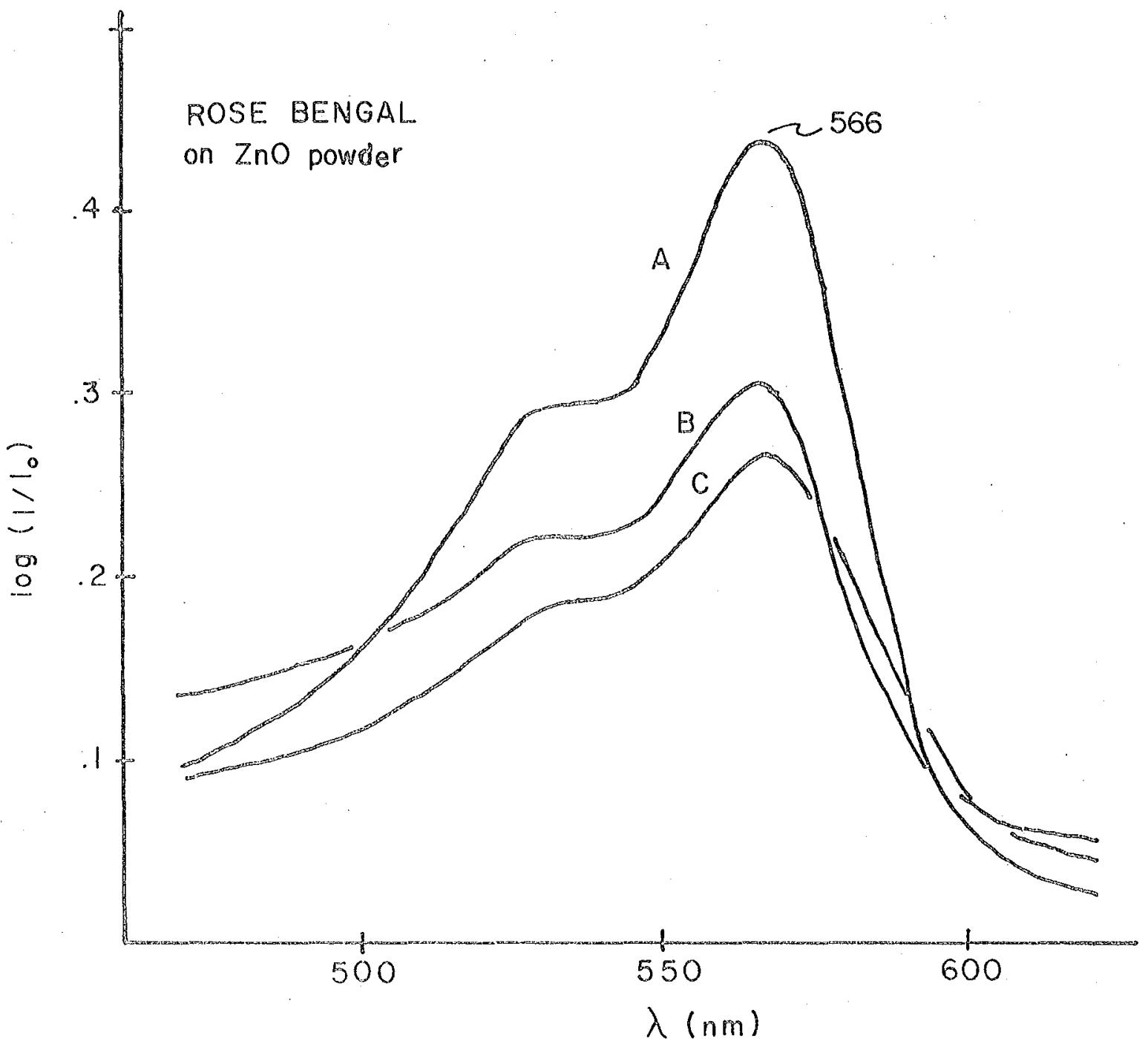

XBL $773-4229$

Figure 12. Spectra taken for the rose benqal covered $Z$ no suspensions using the Cary scattered transmission attachment. These spectra $v$ correspond to points on the adsorption isotherm of figure 11 . 


\section{0}

pair rhodamine $\mathrm{B} / \mathrm{ZnO}$ was selected for this experiment. The resistivity of the Li doped $\mathrm{ZnO}$ was too great for use in electrochemical systems so that higher conductivity crystals had to be found for use as substrates for these adsorption studies.

5. Rhodamine on undoped ZnO single crystals

Optical density measurements of the dye adsorbed at less than monolayer coverage of a single crystal surface were made by alignment of four crystal faces (each with an adsorbed dye layer) in the light path of a Cary 118 spectrophotometer. Two special ce11s were constructed, each with four crystals, so that the use of one as a reference cell in the spectrophotometer resulted in the cancellation of the extrinsic, impurity adsorption of $\mathrm{ZnO}$. Thus, the absorption spectrum of the Rhodamine $B$ adsorbed on the surfaces of the sample cell could easily be recorded.

The crystals used were in the form of cross sectional cuts from a large hexagonal prism with a diameter of $5 \mathrm{~mm}$. A crystal cleaved perpendicular to the main, or " $c$ " axis, the orientation of the cut described here, will expose two different faces, one $\mathrm{Zn}(0001)$, and the other $0(000 \overline{1})$. The crystals were cut with a string saw set at a thickness of $250 \mu$. After grinding with $9 \mu$ SiC grit, both sides of the crystal were polished with $0.3 \mu$ alumina until an optical surface was obtained. The final thickness was estimated at about $150 \mu$.

Real surfaces were created from these polished faces through the use of $85 \% \mathrm{H}_{3} \mathrm{PO}_{4}$ as an etching agent. The crystals were mounted with beeswax on a glass slide so that only one face was exposed; the faces then could be separately etched. The $\mathrm{Zn}$ face was etched for $5 \mathrm{~min}$ in the phosphoric acid, then rinsed witb distilled water and dipped in $0.1 \mathrm{M}$ $\mathrm{HCl}$ for $30 \mathrm{sec}$ to remove any phosphates. For the (.0001) face the $\mathrm{H}_{3} \mathrm{PO}_{4}$ 
is about $1 \mu / \min .^{27}$

Since the 0 face is more vigorously attacked by the $\mathrm{H}_{3} \mathrm{PO}_{4}$ than the $\mathrm{Zn}$ surface, a different etch procedure was required for its preparation due to the rapid accumulation of phosphates. In this case the crystal was exposed to $\mathrm{H}_{3} \mathrm{PO}_{4}$ for $2-3 \mathrm{sec}$ with the resultant phosphates being removed by immersion in the $0.1 \mathrm{M} \mathrm{HCl}$ and a subsequent rinse in distilled water. This was repeated until the magnitude of the etch scratches on the surface was comparable to that of the $\mathrm{Zn}$ face. A portion of the 0 face of several test crystals was covered with wax and the remaining parts were etched as described. When the wax was removed, a step was visible between these two regions, indicating that more than $5 \mu$ of the $(000 \overline{1})$ face had been removed through this procedure.

Each crystal was mounted on a teflon circle over a hole $4 \mathrm{~mm}$ in diameter through which the spectrometer beam passed. They were oriented with the (0001) surface outwards. RTV silicone-rubber glue was used to secure the crystals around the edges.

The teflon circles were inserted in glass tubing cut so that each crystal face was accesible (Fig. 13). When the cell was placed on end with the Zn faces up, the dye solution could be deposited on the surfaces through the apertures in the surrounding glass.

About 1-2 $\mathrm{ml}$ of an aqueous dye solution were deposited on the $\mathrm{Zn}$ surfaces of the cell designated the sample cell. Rate studies showed that 20 min were required for the system to attain equilibrium. The cells were then placed in the spectrophotometer and spectra were recorded. An example of the baseline of the sample cell. without adsorbed dye can be seen in figure 14a. In the spectral region from $450 \mathrm{~nm}$ to $700 \mathrm{~nm}$, the proper orientation of crystals resulted in a baseline flat 


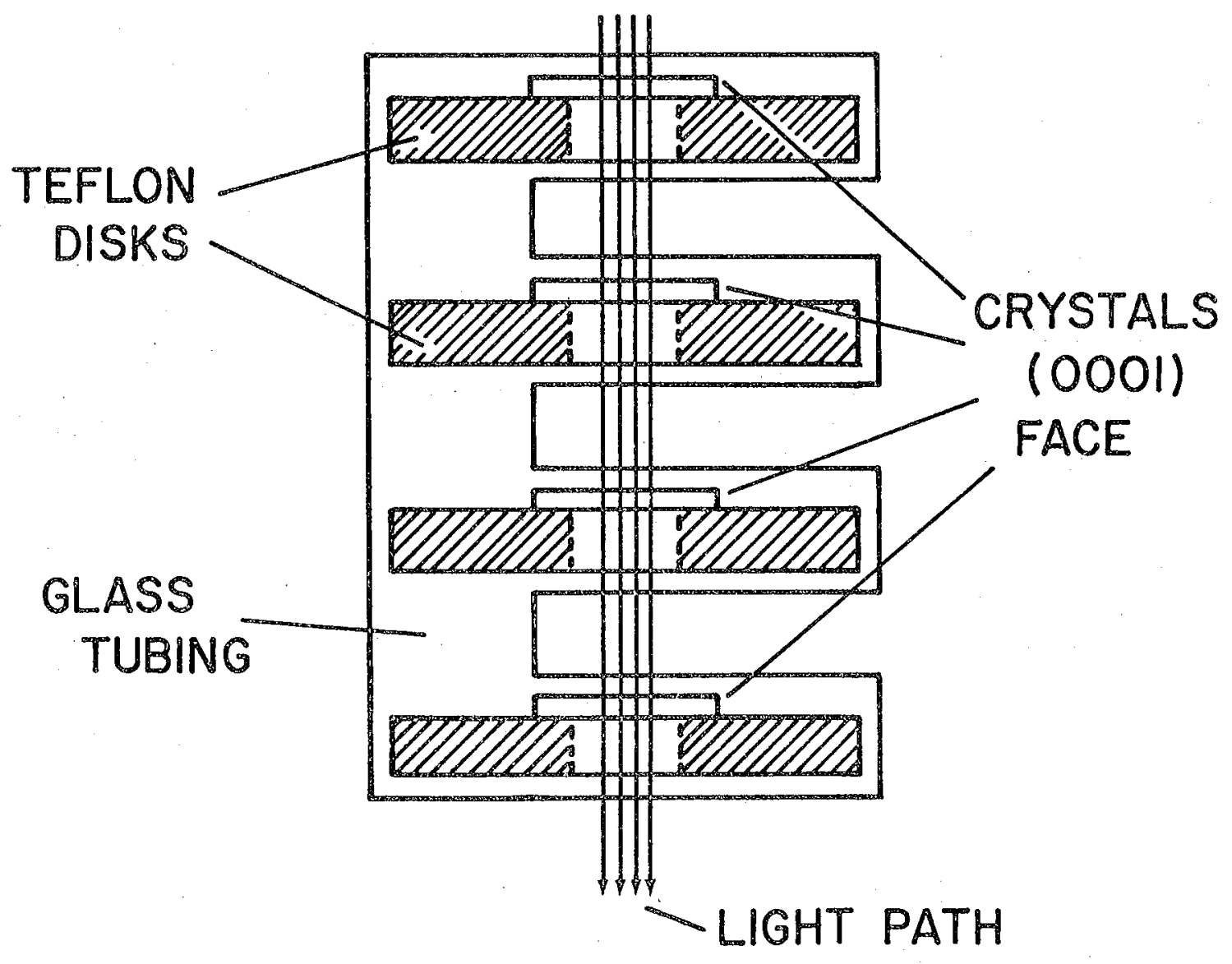

XBL773-4249

Figure 13. A glass cell was constructed for the rhodamine B $\mathrm{ZnO}$ adsorption studies. 
to within .003 .

Solutions of rhodamine B were prepared using doubly distilled water as the solvent. All solutions were adjusted to $\mathrm{pH} 6.5$ and the experiments performed at room temperature, $21^{\circ} \mathrm{C}$. The dye was adsorbed onto the $\mathrm{Zn}$ faces of the sample cell from solutions ranging from $1 \mathrm{x}$ $10^{-5}$ to $6 \times 10^{-4} \mathrm{M}$. Examples of the absorption spectra of the adsorbed dye are compared with solution spectra in figure $14 \mathrm{c}$. The flat baseline allows observation of a shift of the monomer maximum from 557 to 562 $\mathrm{mm}$ as the dye concentration of the solution from which it is adsorbed ranges from $1 \times 10^{-5}$ to $6 \times 10^{-4} \mathrm{M}$. In figure $14 \mathrm{c}$ is a plot of the optical density of the monomer peak versus the concentration of the dye in solution with which the surface is in equilibrium. 


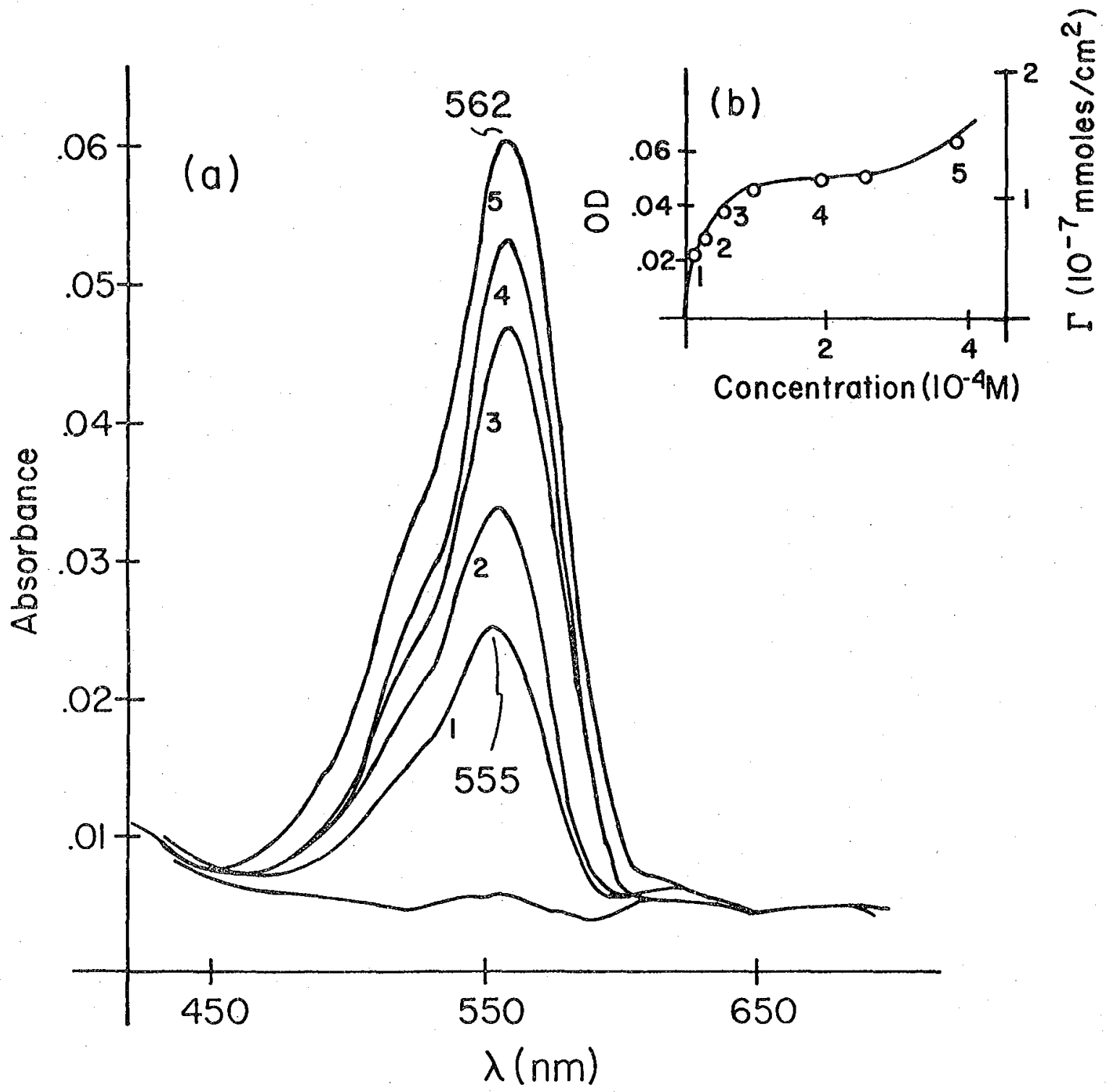

XBL 773-4253

Figure 14. (a) The absorbance of adsorbed rhodamine $B$ on undoped $\mathrm{ZnO}$ is presented at various surface concentrations of the dye. The baseline has been flattened to within .0003 . (b) The OD of the maximum has been plotted as a function of solution concentration with which the dye layer is in equilibrium. 


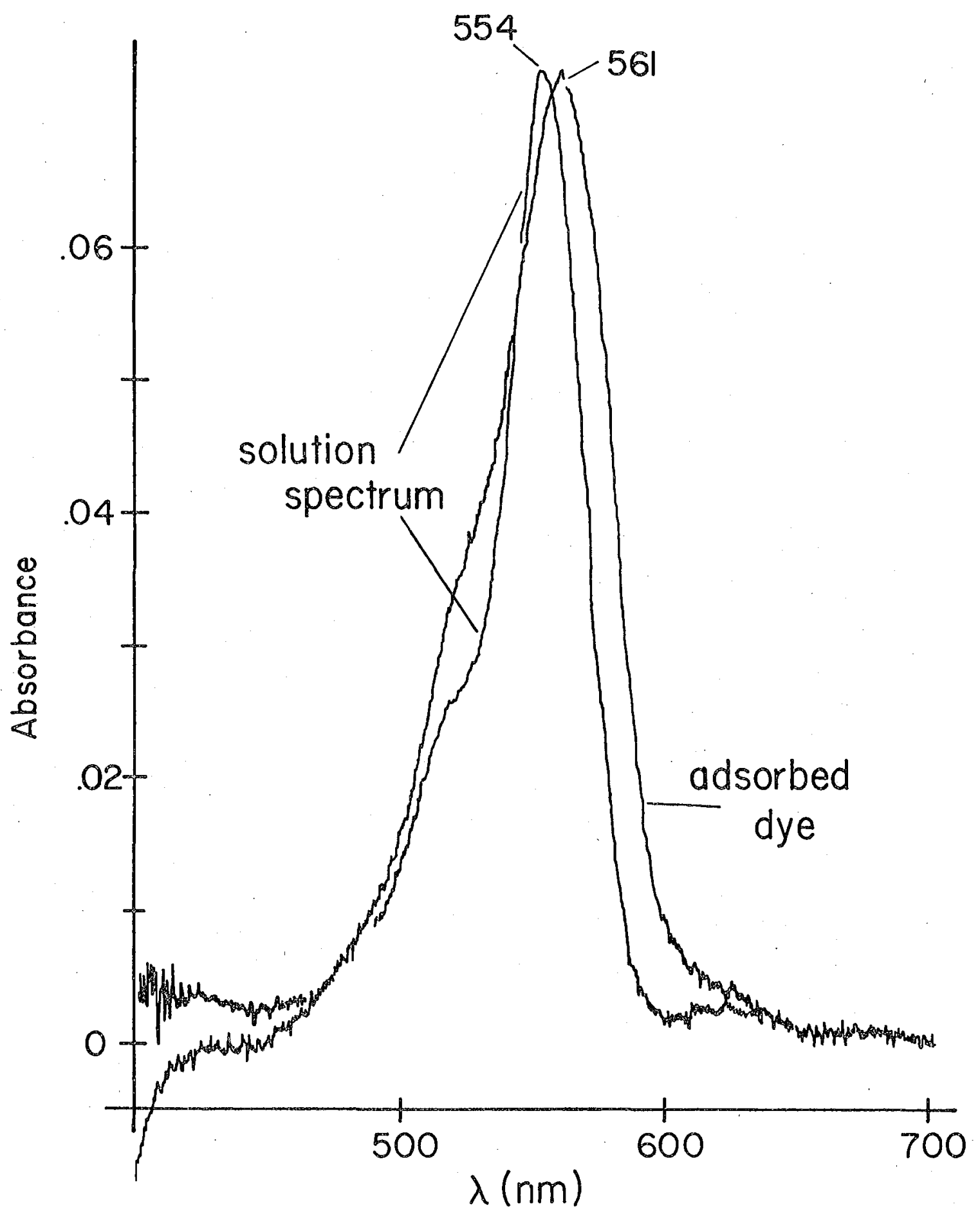
Figure 14c: The absorbance of a $6.3 \times 10^{-7} \mathrm{M} \quad$ XBL $773-4252$
solution of rhodamine $\mathrm{B}$ in water and of the dye adsorbed on ZnO from a $4 \times 10^{-4} \mathrm{M}$ solution are compared. 


\section{E. ANALYSIS}

A cursory comparison of the absorbance of these sensitizing dyes adsorbed on ZnS and ZnO with their solution absorbance will reveal that there are only minor differences between them. The relative concentrations of the monomer and dimer species may change in going from solution to surface, but no new transitions appear upon adsorption to change the envelope of the absorption sepctrum. The most significant difference is a red shift of the absorption maxima from their corresponding energies in solution.

With only these small changes in the excitation spectra, it is safe to conclude that no major modification of the electronic structure of the chrompohore results from adsorption. Although this is not always the case with sensitizing dyes, 28 the interaction between the chromophoric functions of these dyes with the underlying substrate must be only a slight perturbation from that between the dye and the solvent from which it was adsorbed.

Beginning with a qualitative discussion of the absorbance spectra, it can be seen from figures $2,3 \& 12$ that rose bengal and rhodamine $B$ adsorb from solution as monomers even when the solution dye is to a great extent dimeric. This preference for monomer adsorption implies that the free energy of adsorption of the monomer, $\Delta G_{a d s}^{m}$, is more. negative than the sum of the free energies of dimerization, $\Delta G^{\text {dim }}$, and dimer adsorption, $\Delta G_{\text {ads }}^{d}$ :

$$
\Delta G_{a d s}^{m}<\Delta G_{a d s}^{d}+\Delta G^{d i m}
$$

For rose bengal and rhodamine $B, \Delta G_{a d s}$ dim has been established to be -3.3 $\mathrm{kcal} / \mathrm{mole}^{29}$ and $-4.4 \mathrm{kcal} / \mathrm{mole}^{30}$ respectively. Consequently, the energy difference between dimer adsorption and monomer adsorption is 

at least $4.4 \mathrm{kcal} / \mathrm{mole}$ for rhodamine $\mathrm{B}$ and $3.3 \mathrm{kcal} / \mathrm{mole}$ for rose bengal.

This can be interpreted on a more physical level through an examination of a model for dimer formation of these two dyes (figure 15).31,32 This picture of the dimer pairs the two molecules through apposition of the chromophoric planes with the attached phenyl groups oriented about $180^{\circ}$ apart. Evidently, adsorption must occur in an orientation that interferes with this dimer formation. Such an inhibition of aggregation ensues if the molecule sits on the surface with its chromophore next to the surface as seen in figure $16 a$.

However, as the surface concentration of rose bengal on $\mathrm{ZnO}$ increases, the magnitude of adsorbed dimer appears to increase. This is manifest in an increase in the ratio of the absorbance of the high energy shoulder to that of the lower energy monomer maximum. This is in contrast to what was observed with this dye on $\mathrm{ZnS}$ and indicates that the inequality of eqn. (1) must not hold at higher surface concentrations. This difference in adsorbability of the dimer can be ascribed to the difference in the surface structure of these two crystals. Both the $\mathrm{ZnS}$ and $\mathrm{ZnO}$ were polished before use; with Zn0 being the softer of the two crystals, more damage is inflicted on its surface lattice structure with this polishing than would be on the $\mathrm{ZnS}$ structure.

The crystal violet spectra reveal a steady increase in surface aggregation with the rise in the surface concentration of the dye. There appears to be a small amount of curvature in the slow climb of the adsorption isotherm of the $\mathrm{ZnO}$ powder which is also present in the single crystal absorbance plot of figure 8 . This is indicative 


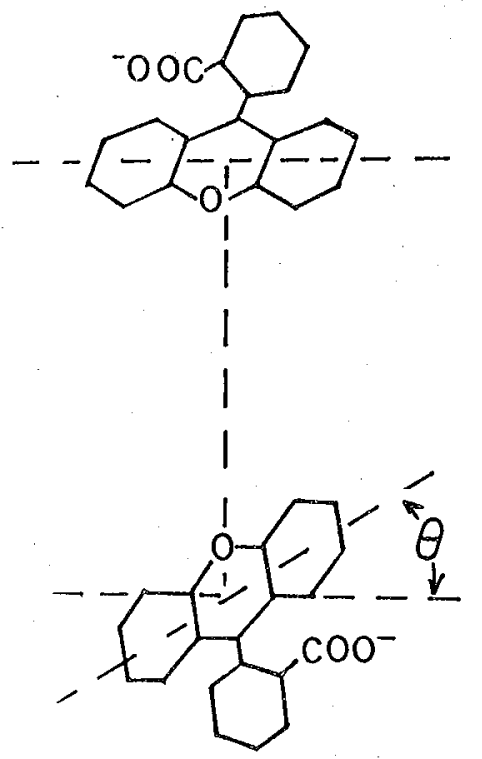

Figure 15. A model for dimer formation of rhodamine $B$ and rose benga 1. The chromophores are paired parallel with the axis of one rotated an angle $\theta$ from the other in the plane. The two phenyl groups are pointed away from one another.

(A)

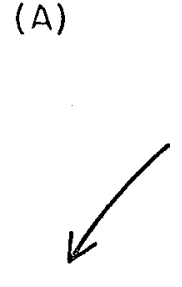

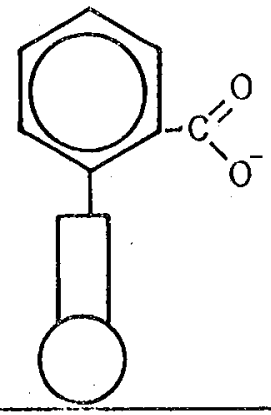

(B)

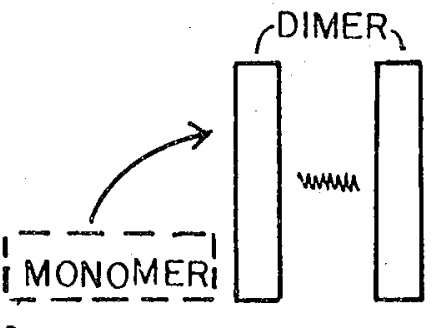

Finure 16. (a) A model for adsorption of rose bengal and rhodamine $B$ that hinders dimer formation on the surface.

(b) Adsorption of the planar

dye probably occurs flat-on at low monomeric coverage. The molecule stands on edge as it interacts to form dimers. 
of a cooperative adsorption which is often observed in systems where adsorption of a planar dye is concerned. As has been determined for adsorption of cyanine dyes on silver halides, 1 the crystal violet most likely adsorbs flat on the surface at low $\theta$ and then stands up perpendicular to the crystal when it dimerizes as parallel molecules at higher $\theta$. It is obvious that at high $\theta$ the inequality of eqn.(1) is reversed:

$$
\Delta G_{\text {ads }}^{m}>\Delta G_{a d s}^{d}+\Delta G^{\text {dim }}
$$

A value for $\Delta G^{\text {dim }}$ is $4.0 \mathrm{kcal} / \mathrm{mole} .^{29}$ An adsorption model for this dye is shown in figure 15 .

A more thorough analysis of these spectra, however, will yield quantitative information about the dye in its adsorbed state and will thereby permit a more detailed description of the dye adsorbed on the crystal surface. No experimental measurement of the amount of dye adsorbed on the semiconductor surface was made because of the small surface area of the crystals. However, a theoretical analysis. 17 of these absorbance spectra can yield the surface concentration, $\mathrm{C}_{\mathrm{S}}$, of the dye. From these theoretical results, adsorption isotherms can be constructed and heats of adsorption determined. This can be accomplished if it is assumed that the transition moment for the excitation is conserved after the dye enters the adsorbed state. This transition moment is defined 33,34 by the quantum mechanical expreșion:

$$
\left|\mu_{\text {Im }}\right|=\int_{-\infty}^{\infty} \Psi_{1} \mu_{x} \Psi_{\text {md } \tau}
$$

where $\Psi_{1}$ and $\Psi_{m}$ are the wave functions for the ground and excited state orbitals of the electron that is energetically promoted. $\mu_{x}$ is the dipole moment operator $\mathrm{r}^{\tilde{r}_{x}}$ where $\tilde{r}_{x}$ is the distance of this 
electron from the center of positive charge.

The square of this transition moment is related ${ }^{35}$ to the rate of excitation, $w$, and the energy density of the radiation field, $\rho$, through the relation:

$$
w=\frac{8 \pi^{3}}{3 h^{3}}\left|\mu \eta_{m}\right|^{2} \rho
$$

This, in turn, can be experssed in terms of the integrated absorption coefficient, $A$, of the molecule undergoing excitation:

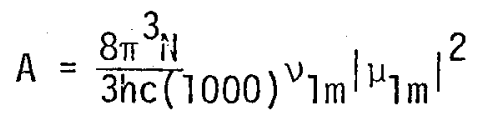

where $v_{1 m}$ is the frequency at the center of the absorption band and $A$ is defined as:

$$
A=\int \alpha(\nu) d \nu
$$

$\alpha(v)$ is the extinction coefficient of the transition and the integral is summed over the frequency range of the absorption.

The transition moment is then proportional to the integrated absorption of the excitation:

$$
\left|\mu_{1 \mathrm{~m}}\right|^{2} \alpha v_{7 \mathrm{~m}} \int \alpha(\nu) \mathrm{d} \nu
$$

Through Beer's Taw, though, the extinction coefficient can be written in terms of the optical density of the dye on the surface:

$$
\alpha(v)=\frac{(O D)}{C_{S}}
$$

With the use of eqns. (2) and (3) the integrated absorption of the dye in solution may be correlated with the optical density of the adsorbed dye:

$$
\int \frac{(0 D)}{\lambda} \mathrm{d} \lambda=\frac{3}{2} \mathrm{C}_{\mathrm{s}} \int \frac{\alpha(\lambda)}{\lambda} \mathrm{d} \lambda
$$

Here an orientation factor $3 / 2$ is inserted to account for the two dimensional arrangement of the transition moments on the surface. With the rearrangement of these terms the surface concentration 
of a dye can be expressed:

$$
c_{s}=\frac{2}{3}\left(\int(O D) \frac{d \lambda}{\lambda} \mid \int \varepsilon \frac{d \lambda}{\lambda}\right)
$$

The task remains simply to calculate these integrals for each dye's solution spectrum and each absorption spectrum recorded for the adsorbed dye. This was done for rose bengal and rhodamine $B$ in water and for the spectra of rose bengal on ZnS and of rhodamine $B$ on Zn0. For the crystal violet spectra and the rose bengal on Zn0 spectra, though, the increasing dimer concentrations precluded such a calculation because two different transitions were involved with relative intensities that changed from spectrum to spectrum. The ratio of high energy to low energy absorption for the two single crystals cases where this integral was calculated changed by at most $20 \%$ during the increase in surface concentration.

These integrals were estimated numerically by summing the value of the term $(\varepsilon / \lambda)$ or $(0 D / \lambda)$ every $6 \mathrm{~nm}$ between 500 and $60 \mathrm{~nm}$. The results are given in Table 1. At the isotherm plateau in figures $7 a$ and $14 \mathrm{c}, C_{\mathrm{S}}$ for rose bengal on $\mathrm{ZnS}$ was determined to be $1.29 \mathrm{x}$ $10^{-7}$ mmoles $/ \mathrm{cm}^{2}$. For rhodamine $B$ on $\mathrm{Zn0} \mathrm{C}_{\mathrm{s}}$ was found to be $1.10 \mathrm{x}$ $10^{-7}$ mmoles $/ \mathrm{cm}^{2}$. Through these calculations, the plots of figures $7 \mathrm{a}$ and $14 \mathrm{c}$ could be transformed into adsorption isotherms as is shown with the ordinates on the right side of these figures.

For the rose bengal/ZnS system this surface coverage yields a surface area of $130 \AA^{2} /$ molecule with a molecule of rhodamine $B$ on Zn0 occupying $150 \AA^{0}$. Examination of the structures of these dyes in the orientations depicted in figures 2 and 3 indicates that $100 \AA^{2}$ would be a reasonable approximation of their cross sectional area. These two isotherms plus that of rose bengal on $\mathrm{ZnO}$ powder 
Table 1. Calculated values of the integrated absorption of rhodamine $B$ and rose bengal (over the range $500-600 \mathrm{~nm}$ ).

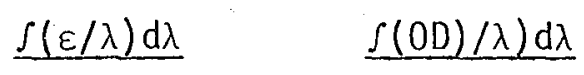

rosebengal

ethanol

ZnS

(point $J$ in

figure $7 b$ )

rhodamine B

ethanol

Zno

(point 5 in

figure 14 b)
$1.623 \times 10^{2}$

$1.301 \times 10^{2}$

$9.5 \times 10^{-5}$

$3.5 \times 10^{-5}$ 
conform to Langmuir type adsorption behavior. In such cases they should be suited to analysis through a Langmuir expression for adsorption from solution onto solids: ${ }^{36}$

$$
\frac{C}{C_{s}}=\frac{C}{C_{m}}+\frac{1}{C_{m}^{B}}
$$

where $C$ is the concentration of the dye in solution in mmoles $/ \mathrm{cm}^{3}$

$C_{m}$ is the surface concentration corresponding to monolayer coverage in $\mathrm{m} / \mathrm{moles} / \mathrm{cm}^{2}$

$B$ is an adsorption constant in $\mathrm{cm}^{3}$ mmole The slope of this line should yield the concentration at monolayer coverage, but its intercept will give a value for $B$ which will assist in determining the heat of adsorption of the dye.

The theoretically established isothernis of figures $7 a$ and $14 c$ have been plotted according to eqn. (4) in figure 17 . In order to plot the isotherm of rose bengal on Zn0 powder, however, a surface area for the powder must be found. This will be estimated through figure 2 to be $100 \AA^{2}$ per dye molecule at the isotherm plateau. The resultant Langmuir plot is also shown in figure 17. The results of this analysis are listed in Table 2.

The cooperative adsorption isotherm of crystal violet on $\mathrm{ZnO}$ powder (figure 11) indicated a change in the adsorption mechanism of the dye. Since this also implies a change in the heat of adsorption with increasing surface coverage, a Langmuir analys is is no longer valid. Consequently, such an interpretation has not been made for this system.

Also included in Table 2 are values for the heat of adsorption of the dyes on their substrates. These estimates can be derived from the Langmuir analysis of these isotherms (figure 17) if a Boltzmann expression is used to describe adsorption at very low concentrations. ${ }^{36}$ 


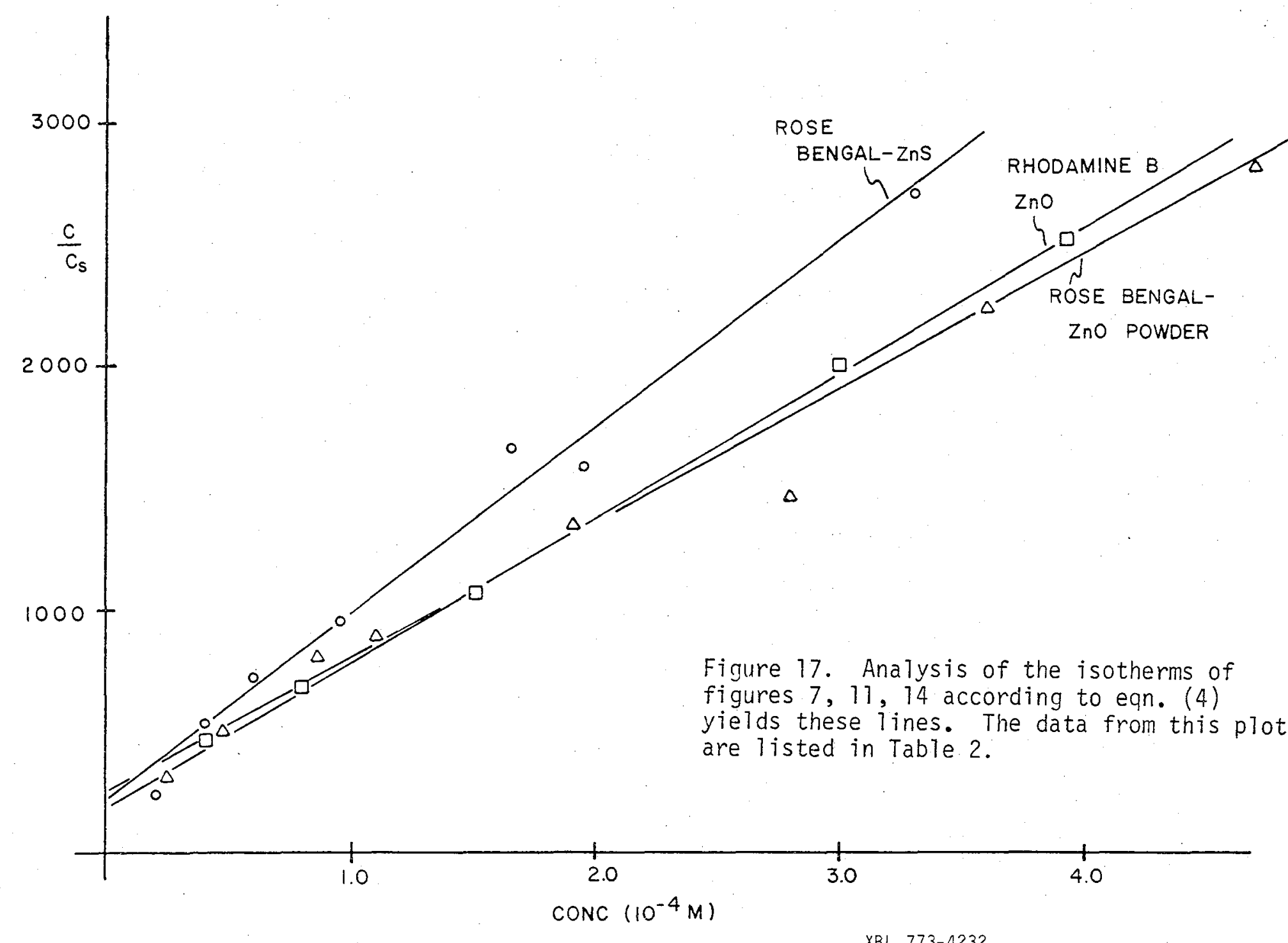

$X B L 773-4232$ 
Table 2. Calculated values for Langmuir isotherms of rose bengal and rhodamine $B$.

\begin{tabular}{|c|c|c|c|c|}
\hline & $\mathrm{ZnS}$ & Zno (powder) & \multicolumn{2}{|c|}{ Zn0 (single crystal) } \\
\hline $\mathrm{Cm}$ & 1.29 & 1.79 & 1.11 & $\times 10^{-7} \frac{\mathrm{mmoles}}{\mathrm{cm}^{2}}$ \\
\hline$\frac{1}{\mathrm{CmB}}$ & $2.1 \times 10^{2}$ & $2.5 \times 10^{2}$ & $3.0 \times 10^{2}$ & $\mathrm{~cm}^{-1}$ \\
\hline B & $3.7 \times 10^{4}$ & $2.2 \times 10^{4}$ & $3.0 \times 10^{4}$ & $\mathrm{~cm}^{3} / \mathrm{mmole}$ \\
\hline $\begin{array}{l}\text { area/ } \\
\text { molecule }\end{array}$ & $130 \AA^{2}$ & $93 \AA^{2}$ & $150 \AA^{2}$ & \\
\hline$\Delta H$ & $5.7-6.3$ & $5.6-6.2$ & $5.7-6.3$ & $\mathrm{kcal} / \mathrm{mole}$ \\
\hline
\end{tabular}


In this limit where $C$ approaches zero, the equilibrium between solution dye and adsorbed dye can be written:

$$
\mathrm{C}=\mathrm{KC} \mathrm{S}_{\mathrm{S}} \cdot 2 \mathrm{r}
$$

where $r$ is the radius of the adsorbed species. Eqn. (4) also has a simpler form as $\mathrm{C}$ becomes very small:

$$
\frac{C}{C}=\frac{1}{C_{m}} B
$$

Combination of these expressions yields:

$$
K=\frac{2 \cdot r}{\mathrm{CmB}}
$$

The $\Delta H$ of the adsorption process can then be determined since

$$
\Delta H=2.3 R T \log (2 r / C m B)
$$

for these dyes, rhodamine $B$ and rose bengal, $2 r$ is taken from models to be $10 \AA$. The intercepts of figure 17 provide values for $1 / C_{m} \mathrm{~K}$.

The results of such a calculation are subject to error owing to the assumptions that have been made for the surface concentrations of the dyes. This error is certainly larger than the uncertainty induced in the estimation of $2 r$ since the assumed surface concentration of the dye can be off by as much as a factor of three. This would introduce an error of +600 calories into the calculated $\Delta H$. It is this uncertainty which appears in Table 2.

F. Discussion

The heats of adsorption found for rose bengal and rhodamine $\mathrm{B}$ on $\mathrm{ZnO}$ and $\mathrm{ZnS}$ substrates are in the range found for sensitizing dyes used in photographic and electrophotographic systems. ${ }^{1,37}$ In these systems, a dye with a $\Delta \mathrm{H}$ of $12 \mathrm{kcal} / \mathrm{mole}$ is considered to be very strongly bound although, indeed, a reaction with a $\Delta H$ of 5-12 $\mathrm{kcal} / \mathrm{mole}$ is not strongly exothermic at all. However, a weak interaction of this size is sufficient to permit electron transfer from the excited dye to the adsorbent. 
Adsorption studies have been reported ${ }^{37}$ where a series of fluorescein dyes, including rose bengal, were adsorbed on Zn0 powders with a known surface area. The heats of adsorption observed for these dyes ranged from 5.0 to $5.9 \mathrm{kcal} / \mathrm{mole}$ with rose bengal exhibiting a $\Delta H$ of $5.9 \mathrm{kcal} / \mathrm{mole}$. This can be compared with the value derived here of $6.2 \mathrm{kcal} / \mathrm{mole}$ where assumptions were made about the surface area of the powder. It appears, then, that these theoretical figures for $\Delta H$ are accurate enough to merit further discussion.

There are two different forces that are considered responsible for adsorption heats of this magnitude. ${ }^{1,38}$ One is a Van der Waals interaction between the adsorbent and the adsorbate, and the other is a coulombic attraction between charged dyes and surface defects with a charge of the opposite sign.

The extent to which coulombic interactions influence the adsorption of these dyes has been determined in several ways. Using erythrosin as an anionic sensitizing dye (which is rose bengal without the four chlorine atoms) and $\mathrm{AgBr}$ as a substrate, it has been reported ${ }^{36}$ that a $10^{6}$ fold increase in the surface $\mathrm{Ag}^{+}$concentration resulted in the heat of adsorption climbing from 6.8 to $9.4 \mathrm{kcals} / \mathrm{moles}$. Furthermore, the extent of adsorption of this dye onto electrophotographic substrates such as $\mathrm{TiO}_{2}, \mathrm{ZnO}, \mathrm{CdS}$, Se, and $\mathrm{S}$ can be diminished through addition of a salt such as $\mathrm{KNO}_{3}$ to the dye solutions. ${ }^{38}$ This increases the dielectric of the solution and diminishes the electrostatic interaction between adsorbate and adsorbent. This decrease in adsorption amounted to as much as 20-40 per cent, but part of this is attributable to competition for surface sites between the dye and the anion of the salt. It can be seen though, that adsorption is due in part to coulomb forces between dye and solid. 
The remainder of the free energy of adsorption has been ascribed to dispersion forces between the crystal lattice and the dye. ${ }^{38}$ The polarizability of the solid is usually greater than that of the solvent from which the dye is adsorbed so that the energy gained by an induced dipole-induced dipole interaction at the surface increases the stability of the adsorbed state relative to the solvated dye. A linear correlation between $\Delta H$ and the polarizability of the fluorescein dyes has been noted. ${ }^{37}$

Consequently, the adsorption of the sensitizing dyes rose bengal, crystal violet, and rhodamine B to semiconductor surfaces can be attribued to a combination of dispersion and coulomb forces.

With these forces active in the adsorption process, a better understanding may be gained as to why the dyes in the adsorbed state are oriented according to the proposed models of figure 16 . Because dispersion forces are so important, the most polarizable portion of the dye molecule will be held closest to the crystal. With its polarizable $\pi$ electron cloud, the chromophore of the dye should be nearest the surface as pictured for rose bengal and rhodamine $B$ in figure 16. This also explains why crystal violet would be held planar on the surface at low $\theta$. Introduction of electrostatic forces into this discussion would appear to place negligible restrictions on these proposed orientations. The charged functions on these molecules can approach an appropriately charged surface site without disturbing the chromophore from its dispersion favored orientation. For example, it has been proposed that the carboxyl function of rhodamine $B$ is attached to acidic surface sites on $\mathrm{ZnO} .{ }^{39}$ Such a restriction still permits a nearly parallel arrangement of the chromophore 
and surface. The charged amino group in crystal violet can be attached at one location and allow both monomer flat-on adsorption and edge-on dimerization.

As the surface coverage increases, it was generally observed that dimer formation increases slightly in some cases as with rose. bengal on $\mathrm{ZnS}$ and dramatically in others as with rose bengal on $\mathrm{ZnO}$. A variety of sites exist at the surface, andat higher surface concentrations a greater number of less favorable adsorption sites would be occupied. The $\Delta H_{\text {ads }}$ for the molecules at high $\theta$ will be more positive than the $\Delta H_{\text {ads }}$ for the initial adsorption of the dye, and the inequality of eqn. (1) will no longer hold. In this case these models of: adsorption of rose bengal and rhodamine B are no longer applicable.

In every case the maximum of the absorbance spectrum of an adsorbed dye is red shifted about $100 \mathrm{~cm}^{-1}$ from its peak in ethanolic or aqueous solutions. This is a general phenomenon that has been observed for all adsorbed sensitizing dyes ${ }^{1}$ and is a manifestation of a greater energy stabilization of the excited state of the dye than of its ground state.

Such a bathochromic shift is observed in solution spectra when the dye's solvent is exchanged for one of higher dielectric. Theoretical explanations 40,41 of this shift account for this phenomenon through consideration of a number of possible interactions of the chromophore with its environment: dipole-dipole, dipole-induced dipole, and induced dipole-induced dipole (dispersion) forces. Dispersion forces constitute the interaction of interest for these dye/semiconductor pairs since they are responsible to a great extent for the adsorption of the dye. Dispersion forces constitute the interaction of interest 
for these dye/semiconductor pairs since they are responsible to a great extent for the adsorption of the dye.

With the exception of the induced dipole-induced portions of the theory, however, most of this work fails to correlate with experimental data. The successes ${ }^{40}$ occur when solvent shifts of solute absorption maxima are related to either the index of refraction of the solvent, $n$, or an expression for the electronic polarizability, $\frac{n_{2}^{2}-1}{n^{2}+2}$ or another function of $n$, the "Bayliss" function, $\frac{n^{2}-1}{2 n^{2}+1}$. All of these correlations are linear; they demonstrate that the macroscopic property connected with a red shift is the index of refraction $n$. On a physical basis this appears true because the response of the solvent to a rapidly oscillating transition moment must occur within the same scale, i.e., the solvent polarization must occur at optical frequencies. The constant which describes the capability of such a response is the optical dielectric constant $\varepsilon_{0}=n^{2}$.

An excellent example of one of these correlations between the solvent displacement of molecular absorption maxima and these functions of $n$ can be found in a paper by West and Geddes. 26 In this work the solvent shifts of a number of cyanine dyes were found to be linear functions $z=\frac{n^{2}-1}{2 n^{2}+1}$. In further experiments of interest here, one of these dyes was adsorbed onto twelve different powder substrates, and the red shift of this adsorbed dye's absorption maximum (or reflectance minimum in this case) was also found to be linear with this function $Z$ where $\mathrm{n}$ was taken to be the refractive index of the underlying solid. The slope of this last relation, however, was approximately one half of the solution line. The data are reproduced in figure 18 .

On a physical level, the use of the index of refraction of the 


\section{0 \\ 347010.6}

adsorbent would imply that the adsorbed dye is completely immersed in a medium of this dielectric. Obviously this is not the case because the dyes are adsorbed at a solid/gas interface. It seems reasonable that a better estimate of the index of refraction of the environment about the dye would be the mean of $n$ for the crystal and $n$ for air: $n_{e}=1 / 2\left(n_{\text {solid }}{ }^{+n_{a i r}}\right)$. If the data of West and Geddes for the adsorbed dye are replotted using this new value for $n$ and $Z$ for each solid, a new correlation is obtained which overlaps the solution line extensively (see figure 18).

This new line cannot be expected to coincide with the olution data because the solvent displacement of the absorption maximum was shown to be attributable entirely to dispersion forces, whereas the condition of the adsorbed dye was not as well defined. To be sure, this indicates that the environmental interactions of the adsorbed dye are determined to a large extent by dispersion forces, but contributions to this red shift may enter through other forces such as charge-dipole effects, and hydrogen bonding that may differ with every substrate.

There are two consequences of these new data. One is that the red shift of the absorption maximum of an adsorbed dye can be used as a measure of the index of refraction and the optical dielectric constant at the substrate surface. Second, the deviations of this new correlations from the solution line probably reflect the influence of forces other than the dispersion interactions.

It was observed that the red shift for rhodamine $B$ adsorbed on Zn0 increased with increasing surface concentrations. This should be expected since close packing of these dyes will change the 


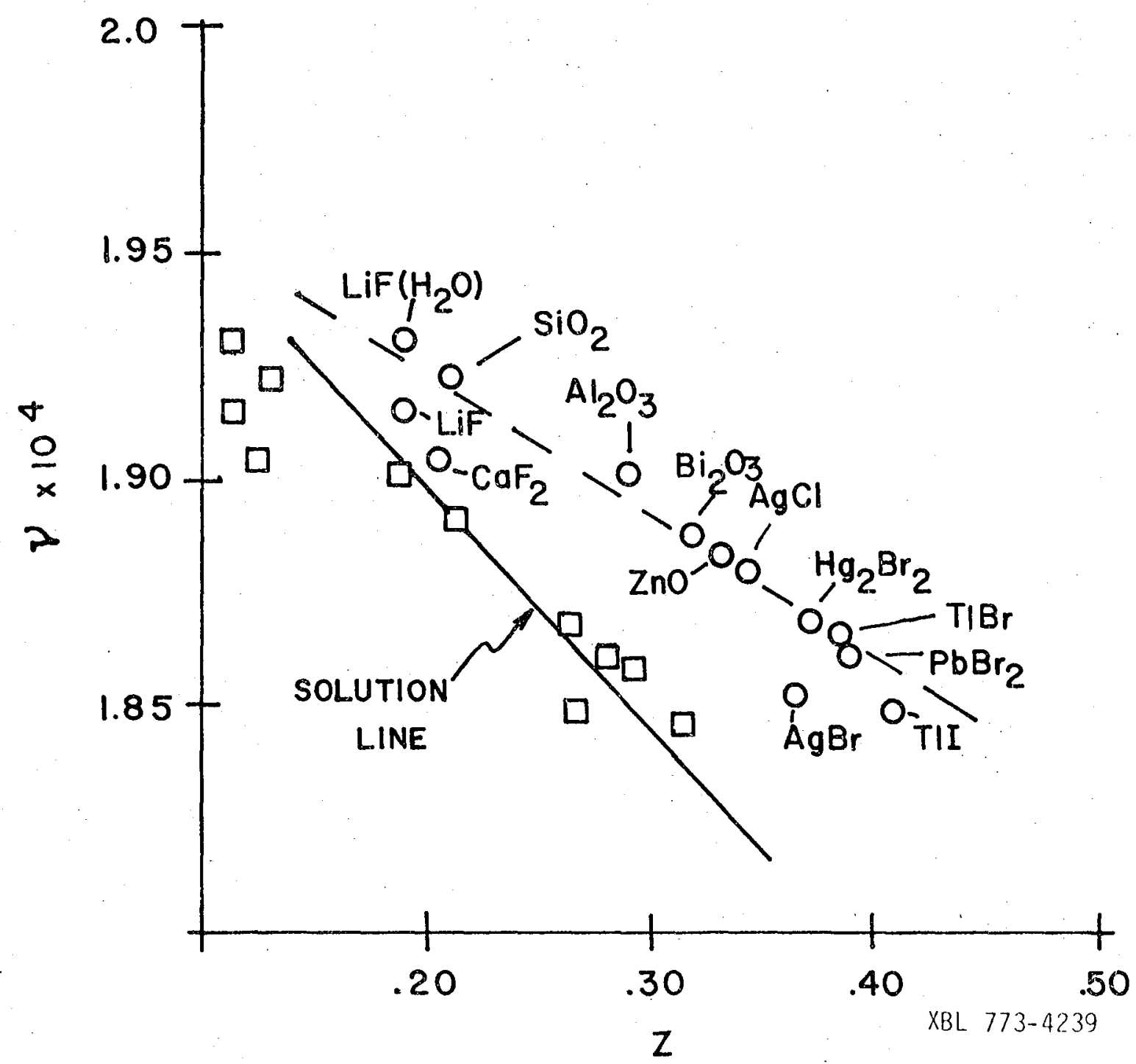

Fiqure 18. The red shift of dye 1,1'diethyl2,2-cyanine cation adsorbed to solid absorbents in relation to the "Bayliss" function is shown by the circles. The red shift described by this dye in solution fits the "solution line". The new correlation where $n=1 / 2\left(n_{\text {sol }}+n_{a}\right)$ is a replotting of the circles and results in the $x$ 's which fall very close to the solution line. 
polarizability of the surface layer from that of air to that of the dye itself. This would result in $n_{\text {air }}$ in the expression for $n_{\text {en }}$ being replaced by the larger quantity, $n_{\text {dye }}$, determined by the properties of the dye molecule itself. 
III. PHOTOOXIDATION OF RHODAMINE B AT ZnO ELECTRODES

\section{A. Introduction}

In this chapter electrochemical techniques will be used to explore dye sensitization of semiconductors. In particular the extensively investigated $^{7,10,18,42}$ dye-semiconductor system of rhodamine $B$ on Zn0 will be examined once again. However, the adsorption techniques developed in Chapter II and the data presented therein permit a much more quantitative study of this system than has previously been possible.

Semiconductor electrochemistry is a complex intersection of solid state physics and electrochemistry. Consequently, some background material in this subject will be provided prior to the presentation of the experimentation with this system. The emphasis in these preliminary discussions will be directed towards the relation between the electronic structures of a molecular species, for example, an adsorbed dye molecule, and the underlying semiconductor substrate. The requirements for electron transfer will be discussed, and the basic experimental set-up of a photoelectrochemical system will be described.

\section{B. Background}

1. Dye-Semiconductor Energetics

(a) Electronic Structure of Semiconductors

A single dye molecule adsorbed onto a semiconductor will have a greatly different electronic structure than its substrate. This difference may be seen in the delocalization of the bonding, or valence, electrons of the molecules in the solid semiconductor over its entire volume. Delocalization results from the ordered arrangement of molecules in a crystal lattice structure to form a periodic potential for the bonding electrons. ${ }^{44-45}$ Such a phenomenon has led to the development of band 
theory to account for the electronic behavior or ordered solids.

According to band theory, ${ }^{46}$ the energies of the valence orbitals of the molecules composing the solid no longer retain the single energy value found for the individual species, but, instead, distribute themselves into bands as is shown in figure 19. As the atoms approach one another during formation of the solid (figure 19) and establish an equilibrium interatomic distance, these bands can be seen to be separated from one another by band gaps where there are no electronic states. If there are an odd number of valence electrons in a molecule which forms a solid, then one half of the most energetic molecular orbitals and, thus, one half of the most energetic band in the resultant solid will be filled, and the solid will be metallic. On the other hand, an even number of valence electrons leads, ideally, to complete occupancy of the most energetic band and to formation of a semiconductor or insulator.

The electrons fill these bands according to fermi statistics so that the probability of an orbital at an energy $E$ being occupied can be expressed:

$$
P(E)=\frac{1}{1+e^{\left(\bar{E}-E_{f}\right) / k T}}
$$

where $E_{f}$ is the chemical potential, or fermi level, of electrons in the solid, and the zero of energy is an electron at infinity in a vacuum. A graph of this function at $\mathrm{T}>0^{\circ}$ is shown in figure 20 in comparison with a density of states diagram of a semiconductor, $D(E)$, which is a measure of the number of electron states in the solid at an energy $E$.

The fermi level is pictured near the center of the band gap between the highest occupied, or valence, band and the lowest vacant, or conduction, band. A precise expression ${ }^{47}$ for position of the fermi level is:

$$
E_{f}=1 / 2\left(E_{c}+E_{v}\right)-1 / 2 k \operatorname{Tin}\left(N_{c} / N_{v}\right)
$$




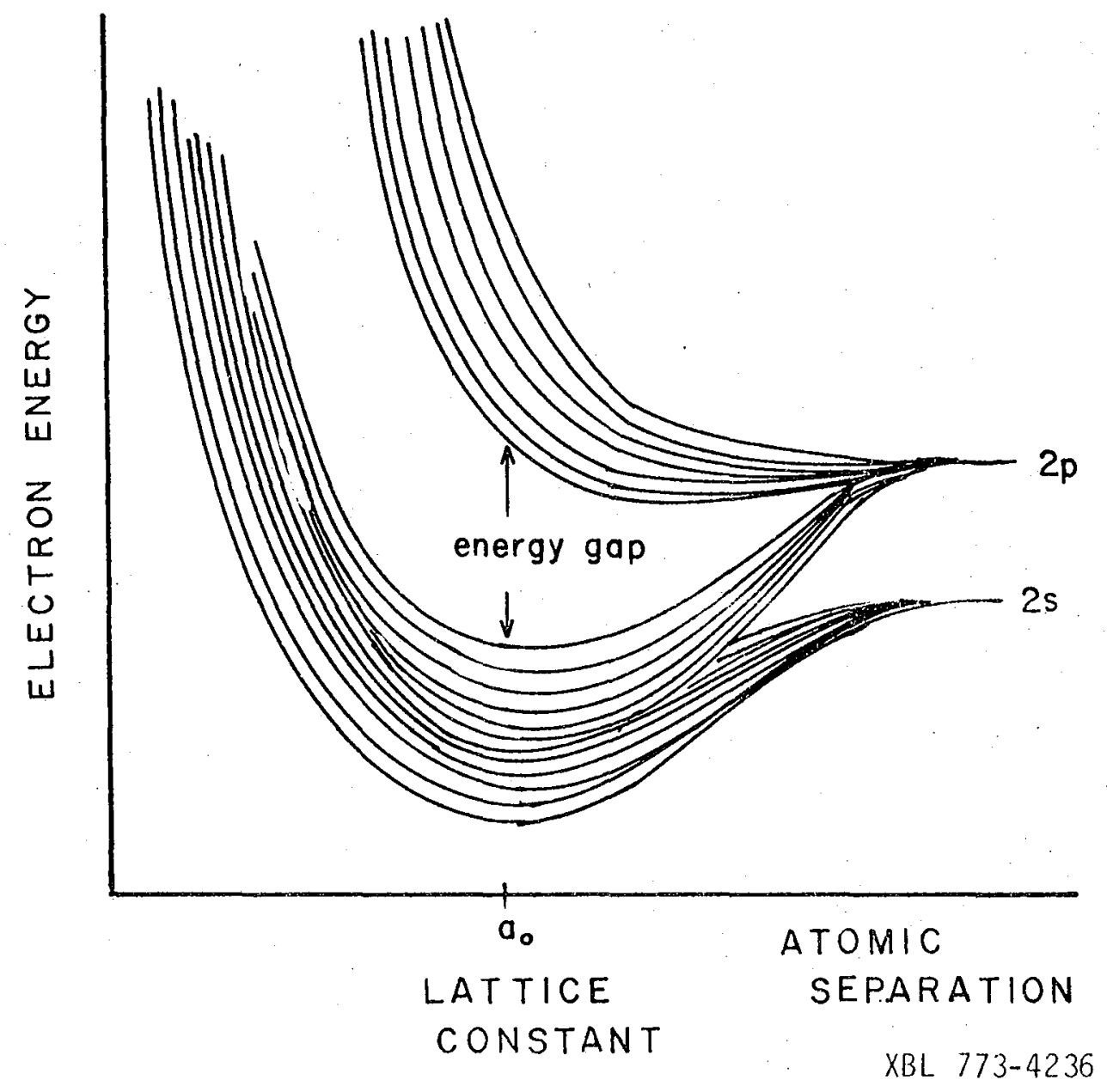

Figure 19. The molecular orbitals of $1+$ carbon atoms spread into bands as the atoms come together to form the diamond lattice. The energy gap represents an energy region where there are no electronic states. For diamond this gap would separate the filled from the unfilled bands. 
where $N_{C}$ is the density of states at the conduction band edge and $N_{V}$ is the density of states at the balence band edge. This is a statistical placement of the chemical potential of the electron and is a consequence of the fact that the probability of occupancy of an orbital at $E_{C}$ is equal to the probability of a vacancy of an orbital at $E_{v}$. This equality exists because thermal excitation of an electron across the band gap results in a vacancy in the valence band.

The electron concentration at an energy level $E$ is given by the product of the density of states and the fermi function

$$
n(E)=P(E) \cdot D(E)
$$

As long as $\left(E-E_{F}\right)>3 k T$, the fermi function may be written in a Boltzmann approximation

$$
P(E)=e^{-\left(E-E_{f}\right) / k T}
$$

At the conduction band edge $E_{C}$, where $D(E)=N_{C}$, the electron population, $n$, would be

$$
n\left(E_{C}\right)=N_{C} e^{-\left(E_{C}-E_{f}\right) / k T}
$$

A similar relation holds for the vacancies left in the valence band by thermal excitation of electrons since these vacancies, or "holes", are mobile charge carriers themselves:

$$
P\left(E_{v}\right)=N_{v} e^{-\left(E_{v}-E_{f}\right) / k T}
$$

The difference between a semiconductor and an insulator is in the size of the band gap between the valence band and the conduction band of the solid. This distinction is made because the conductivity of an intrinsic semiconductor is determined by the thermal excitation of electrons from the valence band across the band gap to the conduction band according to eqns. 5 and 6 . If this band gap is larger than 3-4 eV then the thermal population of the conduction band with electrons will be negligible and the material is called insulating. ${ }^{44}$ However, it is evident that the 


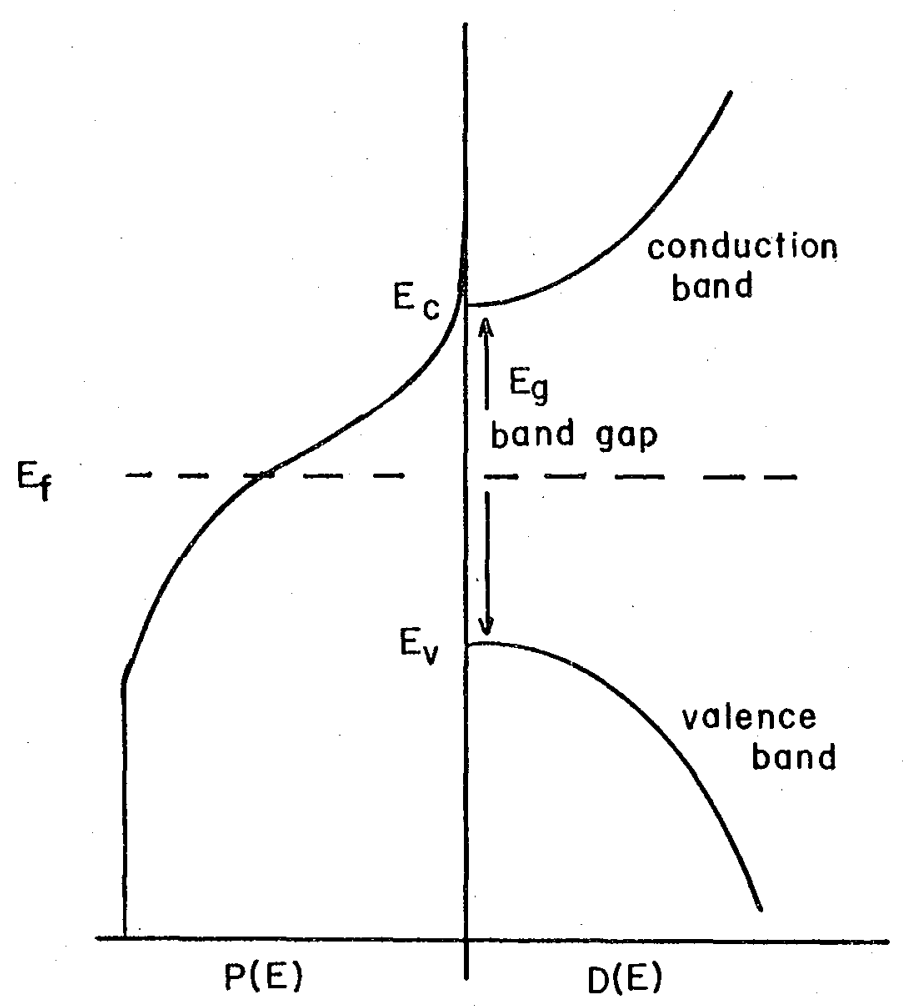

FERMI FUNCTION DENSITY OF STATES

Figure 20. The Fermi function on left is equal to $1 / 2$ at an electron energy in the center region of the bandgap of a semiconductor. electron

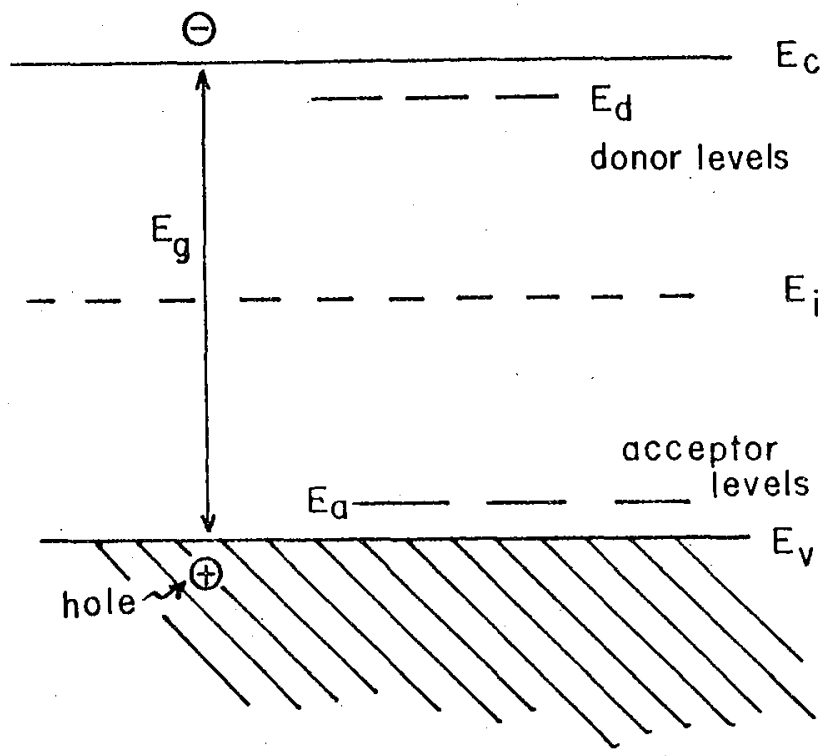

Figure 21. An electron energy diag̣ram in which the photoelectric work function of a semiconductor has an energy $E_{v}$. $E_{i}$ is the Fermi level of the intrinsic material. $F_{D}$ and $E_{A}$ are the energy levels of donors or acceptors in the lattice. 
line between insulators and semiconductors is not distinct and is in general arbitrarily drawn.

Compared with intrinsic silicon and germanium with band gaps of 1.1 and $.76 \mathrm{eV}$, intrinsic $\mathrm{Zn0}$, a wide band gap semiconductor of $3.2 \mathrm{eV}$, conducts very poorly indeed. However, the conductivity of wide band gap compounds can be increased through introduction of impurity atoms, such as Zn or In into $\mathrm{ZnO}$, which can donate electrons to the conduction band of the host lattice and thereby induce "n type" conductivity. Such impurities are known as donors. Other foreign atoms in a lattice can trap electrons from the valence band and leave positive holes in the electronic structure of the valence band. Solids in which the conductivity is determined by holes are known as "p.type" conductors.

The representation of donor or acceptor state in an electron energy diagram (figure 21) depends on the ease with which the impurity is ionized to produce charge carriers. An easily ionizable donor is represented by a level, $E_{d}$, close to the conduction band just as an easily filled acceptor is denoted by an energy level, $E_{a}$, near the valence band. Those impurities more difficult to ionize have levels more distant from the appropriate bands.

It is obvious that the presence of these impurities in the semiconductor will affect the chemical potential of electrons in the solid. In the absence of impurities the chemical potential of electrons, the fermi level, $E_{f}$, is given by eqn. 5 . In the presence of $N_{d}$ completely ionized donors, however, the fermi level will be given by this rearrangement ${ }^{47}$ of the Boltzmann expression:

$$
E_{f}=1 / 2\left(E_{c}+E_{d}\right)-1 / 2 k T \ln \left(N_{c} / N_{d}\right)
$$

An analogous expression can be written for acceptors:

$$
E_{f}=1 / 2\left(E_{v}+E_{a}\right)+1 / 2 k T \ln \left(N_{v} / N_{a}\right)
$$


Once the fermi level approaches within $3 \mathrm{kT}$ of a band edge, though, the Boltzmann approximation is not an accurate representation of the fermi function and cannot be used.

(b) Energy Relationships between Dye and Semiconductor

An exchange of a charge carrier between an adsorbed dye molecule in an excited state and an underlying semiconductor can be observed only when certain energetic relationships exist between the dye and solid. The electronically excited dye has two reactive energy levels: the level at which the excited electron is found, $E_{D}$, and the half vacant molecular orbital from which it originated, $E_{D}$. In order to obtain charge transfer, these two levels must overlap 48 with the electronic structure of the semiconductor in one of the two ways diagrammed in figure 22.

When the vacancy in the ground state of the excited dye molecule overlaps with the valence band, the hole can be injected into the solid, and the dye is reduced. Oxidation of the dye occurs when the electron in its first excited state can tunnel into the conduction band (figure 22). However, if the excitation energy of the dye is greater than the energy of the band gap of the underlying semiconductor, then charge transfer will be precluded by an efficient quenching of the excited dye through resonance energy transfer with the solid.

Thermodynamically, it is apparent that the photooxidation of a dye molecule at a semiconductor surface requires that the chemical potential, $E_{f}$, of electrons in the solid must be more negative than that of the electron in the excited dye molecule. This latter quantity, designated by the redox potential $E_{D^{*} / D^{+}}$, is defined by the reaction:

$$
D^{*} \longrightarrow D^{+}+e^{-} \quad E_{D^{*} / D^{+}}
$$

and will assume greater significance in later electrochemical discussions 


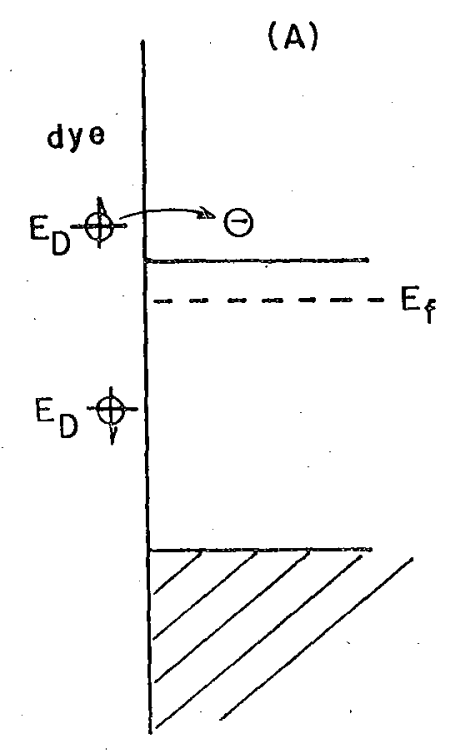

(B)

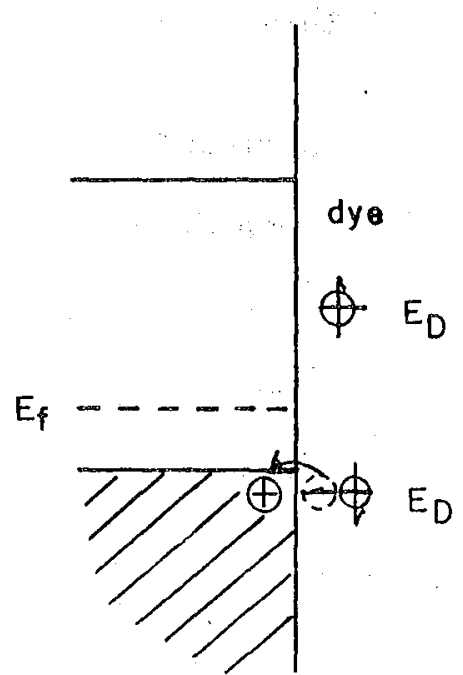

Figure 22. There are two possible modes of electron transfer through dye sensitization. In (a) an electron is injected into the conduction band whereas the process in (b) requires hole injection into the valence band.

(A)

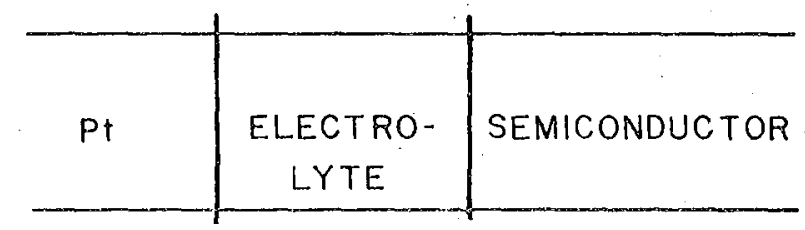

Fiqure 23. Diagrams of semiconductor electrochemical circuit (a) with $\mathrm{Pt}$ and semiconductor immersed in electrolyte and externally shorted.

(B)

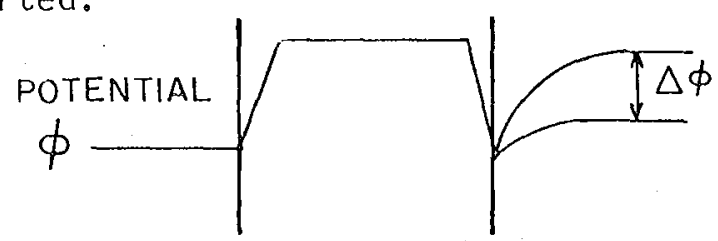

(b) Applications of potential $\Delta \phi$ to system

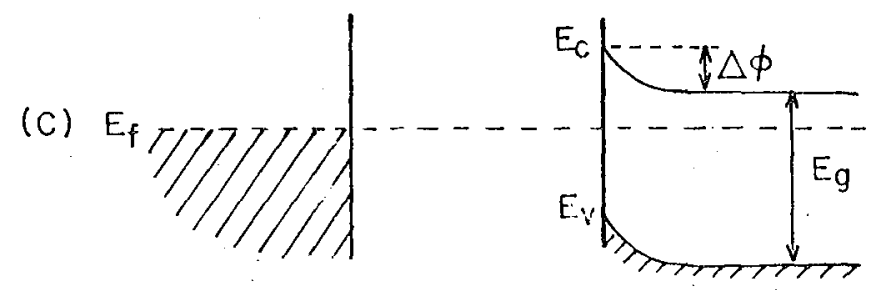

XBL $773-4238$ results in almost the full $\wedge \phi$ being felt by the semiconductor. This results in the band bendina of $(c)$. 
of this phenomenon.

\section{Semiconductor Electrochemistry}

Use of electrochemical techniques to investigate sensitization of semiconductors was first introduced by Gerischer and Tributsch! 12, 13, 14 prerequisite to this initial work, however, was the development of the basics of semiconductor electrochemistry. ${ }^{49,50,51}$ To provide some of these basics as background, a few important aspects of semiconductor electrochem-istry will be presented here as they apply to dye sensitized electron transfer. However, only the energy configuration of figure $22 a$ will be considered in this discussion because the succeeding experiments deal solely. with the photooxidation of dyes at $\mathrm{n}$ type semiconductors.

If this semiconductor is used as an electrode in an electrochemical cell, a positive voltage can be applied to the crystal in an amount sufficient to draw off all of the electrons injected by the excited dye. Given an electrical circuit consisting of a moderately doped semiconductor, an aqueous electrolyte, and a Pt wire as in figure 23, an applied voltage $\Delta \phi$, will not fall evenly across the three elements. If the electrolyte concentration is greater than 3 millimolar (about $10^{18}$ charge carriers/cc), the most resistive element of the circuit will be the semiconductor (about $10^{16}$ charge carriers/cc), and it will bear the entire potential drop. 52 The effect of such a bias on the band energies is shown in figure 23 as band bending in the surface region. In the bulk of the semiconductor, the electron concentration in the conduction band is given by

$$
n_{\text {bulk }}=N_{C} e^{-\left(E_{c}-E_{f}\right) / k T}
$$

However, the band bending in the surface region imposes an additional energy requirement, $\mathrm{e} \Delta \phi$, for population of the conduction band at the surface. Consequently, the electron concentration at the surface may be 
written:

$$
n_{s}=N_{c} e^{-\left(E_{c}-E_{f}\right) / k T} \cdot e^{-e \Delta \phi / k T}=n_{b u 1 k} e^{-e \Delta \phi / k T}
$$

Thus the surface region across which this potential drop, e $\Delta \phi$, occurs, is effectively depleted of a11 electrons creating a space charge region consisting only of the immobile, positively charged donor atoms.

It should be emphasized that this potential drop falls almost entirely within the semiconductor so that the energy difference between $E_{C}$ at the surface and $E_{D^{*}}$ for the dye (see figure 22) does not change with polarization of the electrode. In contrast to metal electrodes, an applied potential has almost no effect on the potential drop across the Helmholtz layer except when the semiconductor becomes degenerate and begins to behave as a metal. This occurs when the fermi level of the semiconductor rises above the conduction band edge, and this band becomes heavily populated with electrons.

This is a critical difference between metal and semiconductor electrochemistry as it implies that the activation energy of the dye photooxidation reaction at the surface is not affected by an applied bias. Only that of the reverse reaction, an electron leaving the electrode, will be changed.

The probability of an electron returning to a dye molecule after injection into the conduction band will depend on the electron concentration in the solid at the surface. This concentration, in turn, is determined through eqn. 8 by the band bending $\Delta \phi$ at the surface so that a large positive bias voltage will insure that none of the electrons leaving the excited dye species will return to the parent molecule. However, above a certain value of the current drawn from this circuit will be limited by the efficiency of injection from the excited state of the dye, and a saturation current will be attained. 
There is another feature of this charge depletion region in the solid which affects current flow through the interface, and that is its capacitive nature. $49,51,53$ on either side of the space charge region are charge carriers, electrons in the semiconductor bulk and ions in the Helmholtz layer of the electrolyte which function as charged plates for what is, in effect, a parallel plate capacitor. The differential capacitance of this region may be defined as $C$ :

$$
C=\frac{d q}{d \phi}
$$

where the derivative $\frac{d q}{d v}$ is to be found from solution of Poisson's equation,

$$
\frac{d^{2} v}{d x^{2}}=-\frac{p(x)}{k \varepsilon}
$$

where $p(x)$ is the charge density; $\varepsilon$ is the dielectric constant of the solid and $k$ is the static permitivity constant.

Integration of this expression for a depletion region (the Mott-Schottky approximation) is straightforward with the assumption that the charge distribution, the density of ionized donors, $N_{d}$, is uniformly spread throughout the crystal:

$$
q= \pm\left( \pm 2 \varepsilon k \int_{0}^{\phi} \int_{0}^{\operatorname{S}} N d(\phi) d \phi\right)^{1 / 2}
$$

where $q$ is the total charge and $\phi_{S}$ is the size of the band bending. Differentiation according to the definition of eqn. 9 leads to a relation between the capacitance of the space charge layer and the potential drop across it:

$$
C=\frac{d q}{d v}=\left(\frac{q^{2} N d \varepsilon \varepsilon o}{2 k T}\right)^{1 / 2}\left\{-1-\frac{e \phi}{k T}\right\}^{1 / 2}
$$

For a real system the capacitance can be measured easily with an impedance bridge. From eqn. 10 a plot of $\left(1 / c^{2}\right)$ against $\phi$ can be seen to be linear with the siope proportional to the donor density $N_{d}$. This is often used as a measure of the carrier concentration in the semicon- 
ductor. 53,54

At a semiconductor-electrolyte interface, the absolute value of $\phi$ is not known because of other potential drops in the circuit (figure 23). However, use of a reference electrode can provide a reference voltage, $\dot{E}_{\text {ref }}$, against which $1 / c^{2}$ can be plotted to obtain $N_{d}$ (see figure 24). At one point $1 / C^{2}$ intersects the abscissa and it can be seen from eqn. 10 that this represents the potential with respect to the reference electrode, where there is only a $60 \mathrm{mV}$ drop across the space charge region. The bands are then effectively flat from the interior of the crystal to the surface. This condition is called the flat band potential, $E_{f b}$, and represents the energy difference between the potential of the reference electrode and the fermi level of the semiconductor:

$$
E_{f b}=E_{f}-E_{\text {ref }}
$$

From eqns. 11 and 7 we can see how the energy level of the conduction band $E_{C}$ can be related to an electrochemical reference electrode:

$$
\left(E_{c}-E_{r e f}\right)=E_{f b}-k T \ln \left(\frac{N d}{N c}\right)
$$

It is at this potential, $\left(E_{C}-E_{\text {ref }}\right)$, and those more negative that oxidative electron transfer occurs via the conduction band of a semiconductor electrode.

Implicit in this discussion of the flatband potential is the assumption that the potential of the reference electrode corresponds to a specific energy in the electron energy diagram of figure 22 . The usual reference point for electrochemical studies is the potential of the standard hydrogen electrode (SHE) which has been set to be zero volts.

These capacitance discussions have assumed, in effect, that an electron in the reference electrode has a defined energy $E_{\text {ref }}$ with respect to an electron in vacuum. ${ }^{10}$ Such an absolute energy scale must then be 


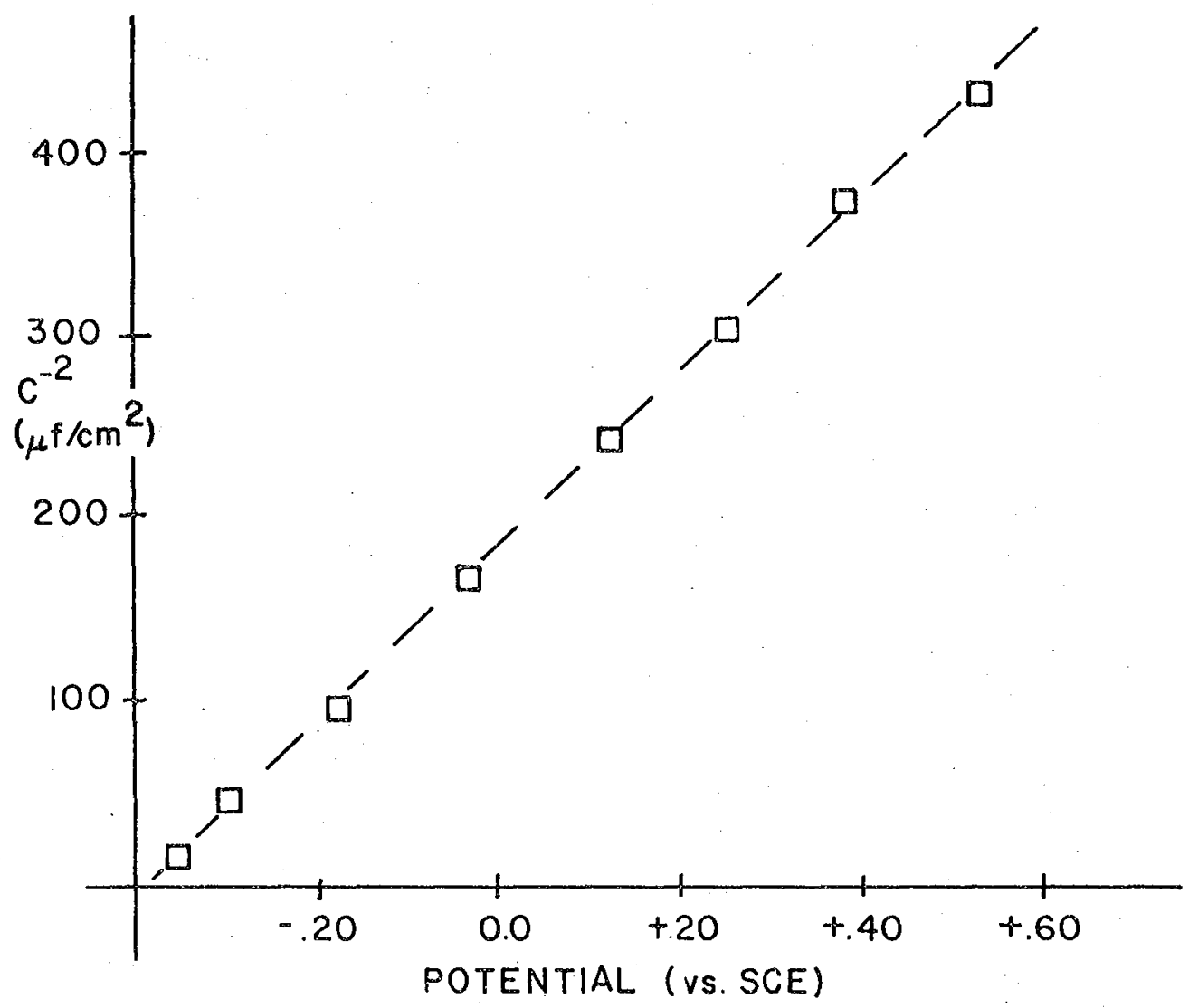

Figure 24. A plot of $(1 / C)^{2}$ against a reference electrode is linear.

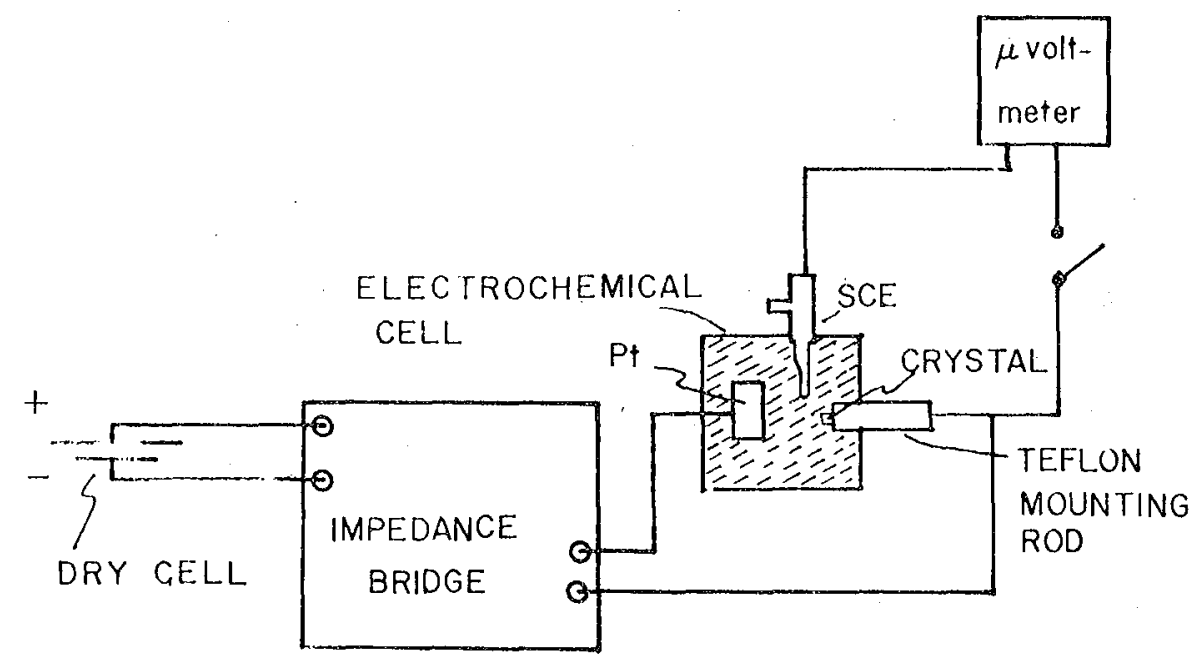

Figure 23. A block diagram for capacitance measurements is shown here. A bias supplied by the dry cell is internally imposed on the circuit by the bridge. 


\section{$00 \% 14710114$}

fully parallel to the conventional electrochemical redox scale, but shift-

ed by the energy $E_{\text {ref }}$ of the SHE. This has been calculated to be $-4.5 \mathrm{ev}^{55}$ so that the relation between the two scales may be written

$$
E=-4.5-e_{0} U
$$

where $U$ is the conventional redox potential.

3. Experimental Apparatus for Photoelectrochemical Measurements

In figure 25 is shown the basic experimental set-up for photoelectrochemical measurements. A light source provided a photon flux which passed through a monochromator and was modulated with a mechanical chopper. The modulated current produced at the semiconductor/electrolyte interface was amplified with a current to voltage converter and detected with a lock-in amplifier. The bias at the semiconductor was maintained with the use of a potentiostat, a counter electrode, and reference electrode.

The electrochemical components consisted of a Wenking potentiostat, an electrochemical cell and a PAR 175 Universal Programmer function generator used for potential sweeps of the electrode. A PAR 181 current to voltage converter was also included in the circuit since the currents were too small (on the order to 10 nanoamps) to be accurately measured by the current meter in the potentiostat.

The electrochemical aspect of the set-up which was used for measurement of photocurrents required the use of three electrodes and a potentiostat. The semiconductor served as one electrode with a Pt wire as a counter electrode. A standard calomel electrode (SCE) served as a third. A simplified schematic for the employment of these three electrodes with a potentiostat is shown in figure 26 . There are two basic circuits involved. One is a potential measuring circuit between the semiconductor and the SCE through which negligible current is drawn. This yields the bias potential of the semiconductor versus a standard reference potential, 


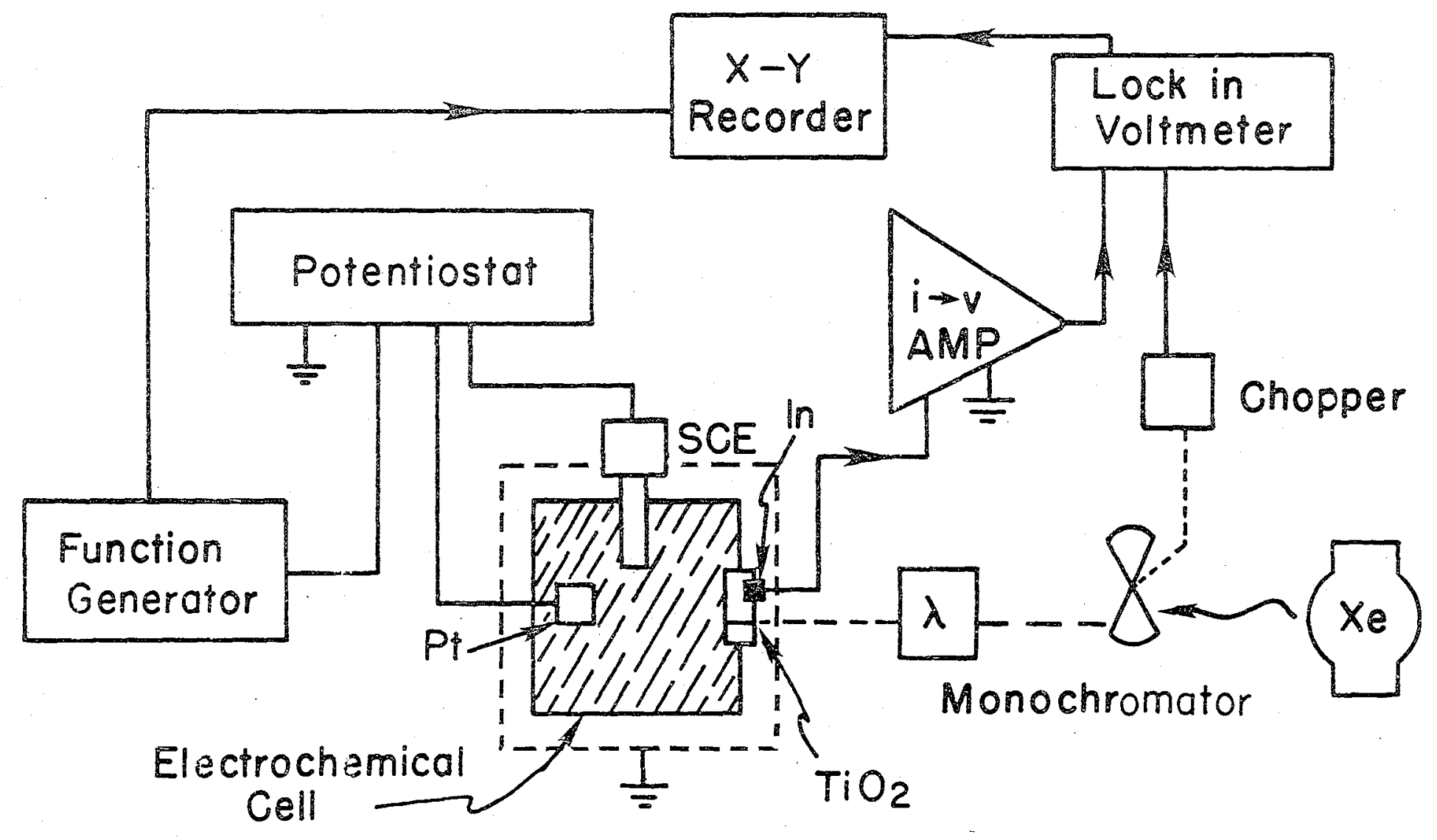

Figure 25. The basic experimental apparatus

used in the measurement of dye sensitized photocurrents. 


\section{$00 \times 14710113$}

$\left(E_{F}-E_{\text {ref }}\right)$. The second circuit through the Pt counter electrode and semiconductor carries the current by which the bias on the semiconductor can be controlled. The desired bias can be selected and applied to the input of the potentiostat with a function generator where it is compared electronically with $\left(E_{F}-E_{r e f}\right)$. If these differ, current is pushed through the Pt electrode and semiconductor circuit until the voltage seen by the potential measuring circuit changes to match the input voltage applied to the potentiostat by the function generator.

Several light sources were used. A high intensity 450 WXe high pressure lamp was employed upon occasion as were several different $150 \mathrm{~W}$ tungsten lamps. The latter were much quieter and served quite well for the rhodamine photocurrent measurements, but they did not have the intensity required for the rapid inducement of a time decay of the photocurrent that was desired in several experiments.

A Bausch and Lomb high intensity monocnromator was used in conjunction with Xe lamp for the recording of action spectra. This was often supplanted with interference filters for very high intensity requirements at specific wavelengths and when relatively high intensity was desired from the 150 W lamps.

Modulation of the light occurred after wavelength selection to ease measurement of the photocurrent. Care had to be taken, however, to avoid chopping frequencies which exceeded the RC time constant of the electrode. 20 Larger crystals had larger capacitances so that the modulation frequencies had to be decreased. Frequencies in the range of 4-12 Hz were used depending on the surface area of the crystal.

A mechanical chopping system was used which had been fabricated in the laboratory. It consisted of a step motor and a control power unit which applied the stepping pulse after reception of a trigger supplied 


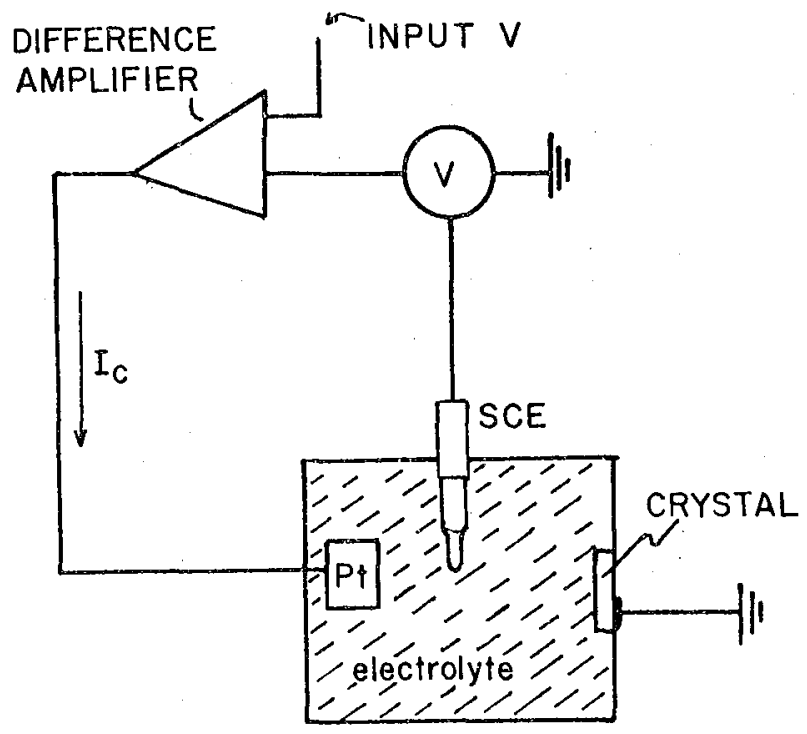

Figure 26. In a potentiostatic arrangement the $\triangle V$ between SCE and the crystal is measured with a voltmeter. This is compared with the desired $\Delta V$, the input voltage, at a summing junction. If they differ, a correction current $i_{C}$ is pushed through the $P+$ electrode and the crystal to ground until they are equal.

(A)

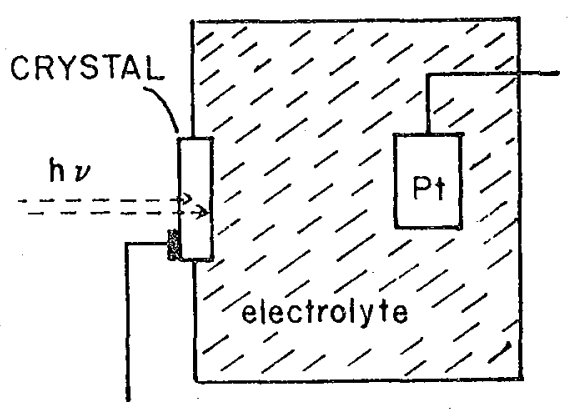

(B)

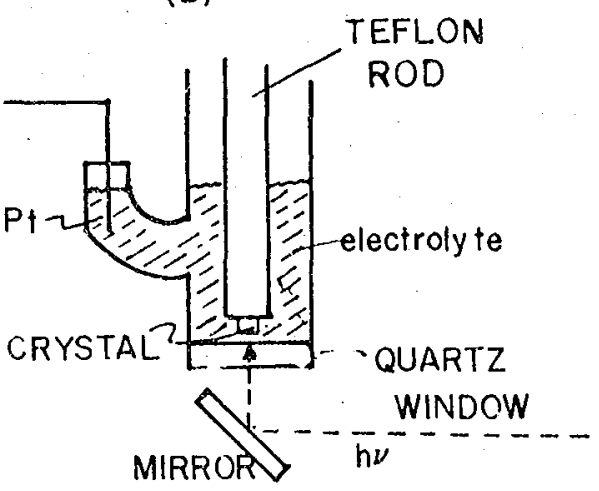

Figure 27. Two configurations were available for $i 11$ umination of the dye/semiconductor surface. One (a) is through backside illumination using the crystal as an optical window; the other (b) allows the crystal to rest on a quartz window through which the light passes. 


\section{$0004 \% 10110$}

by a Data Pulse 1000A pulse generator.

The PAR current to voltage converter has a 10-volt full scale output for ranges from $10^{-9}$ to $10^{-4}$ amps. There are two outputs so that one could be fed into the lock-in amplifier and the other directly to a recorder if a $D C$ measure of the photocurrent was desired. The HR-8 lockin amplifier demodulated the output of the 181 with the aid of a reference signal from the chopping apparatus.

The crystals used as electrodes have band gaps greater than $3.0 \mathrm{eV}$ and were, therefore, transparent to the visible spectrum. Consequently, they could be used as optical windows for illumination of the dye/semiconductor surface. This arrangement is included in figure 27a. Another configuration of the electrode is shown in figure $27 \mathrm{~b}$ where the crystal is mounted on a teflon rod and allowed to rest on the quartz window through which the actinic light is reflected by a mirror. The distance between the crystal and the window was small enough to prevent significant absorption of the light by the intervening dye in solution, but not small enough to impede electrolytic conduction.

Capacitance measurements 53 of crystals were taken using the set-up of figure 28. A General Radio $1650 \mathrm{~A}$ impedance bridge at $1 \mathrm{kHz}$ was used with a Kiethly 151 microvoltmeter which measured the potential of the crystal with respect to a saturated calomel electrode. A $24 \mathrm{~V}$ dry cell supplied the bias voltage to the impedance bridge which imposed it internally across the circuit of the semiconductor electrode and a large surface area Pt electrode of about $10 \mathrm{~cm}^{2}$. 


\section{Rhodamine B Sensitization of $\mathrm{ZnO}$}

1. Introduction

A quantitative examination of a dye sensitized system is a logical followup to the qualitative explorations that have marked the investigations in this field to date. $7,10,12-14,18-20,56-60$ This is possible only when the techniques of Chapter II have been established, and the resultant data is available for correlation with photoelectrochemical results from the same dye-semiconductor system.

Such an examination has been attempted here with the dye rhodamine $B$ at $\mathrm{ZnO}$ single crystal electrodes. The photocurrent data from the electrochemical measurements can be compared with absorbance measurements from Chapter II. As a result, the quantum efficiency of electron transfer can be established as a function of the surface coverage of the dye. Further use of adsorption data permits a quantitative comparison of the ability of reducing agents to increase the photocurrent in a process termed "supersensitization".

Additionally, the time decay of the photocurrent can be analyzed using classical kinetics. This yields a figure for the surface concentration of the dye which can be compared with that derived from adsorption studies.

\section{Materials}

The undoped $\mathrm{ZnO}$ single crystals and purified rhodamine B of Chapter II were used in these experiments. The crystals were contacted with conductive silver epoxy on the (000T) face to make electrodes. If the crystal was to be used as an optical window, the center of the (000T) face was left free of epoxy. The contacted crystal was mounted on teflon with an inert silicone rubber glue, Dow-Corning 3145 RTV. Capaci- 
tance measurements gave a donor density of $2.3 \times 10^{16} / \mathrm{cc}$ and a flatband potential of $-0.4 \mathrm{~V}$ (SCE).

For additional adsorption studies, the Li doped ZnO plate crystal described in Chapter II was also used in adsorption studies. The conductance of these crystals was not measured but can be calculated as shown in Appendix II.

\section{Results}

(a) Comparisons with Adsorption Data

(i) Action Spectra

At a positive applied bias sufficient to draw off all injected electrons, the current will vary with the wavelength of the light incident on the dye-semiconductor interface. An example of such an action spectrum for rhodamine $\mathrm{B}$ at an $\mathrm{Zn} 0$ electrode is given in figure 29 . The $a b-$ sorbance of the dye on the crystal surface is also included in this figure. It is taken from the data of figure 14b. For this measurement the crystal was maintained at a bias of $+1.0 \mathrm{v}$ (SCE) and exposed to light through backside illumination from the Xe lamp modulated at $11.6 \mathrm{hz}$. The bandwidth of incident light for both spectra was $0.5 \mathrm{~nm}$.

The photocurrent action spectrum of rhodamine $B$ on $\mathrm{ZnO}$ reflects the absorbance spectra of the adsorbed dye. Aside from minor differences, the envelope of the two curves (figure 29) are the same, showing that the efficiency of electron transfer is constant over the spectral range of the dye. Therefore, as expected, the higher energy excitation represented by the absorption shoulder must then decay quickly to the first excited singlet state from which oxidation occurs.

The absorbance spectrum is red shifted from the action spectrum by several nanometers which cannot be explained in terms of the average in- 


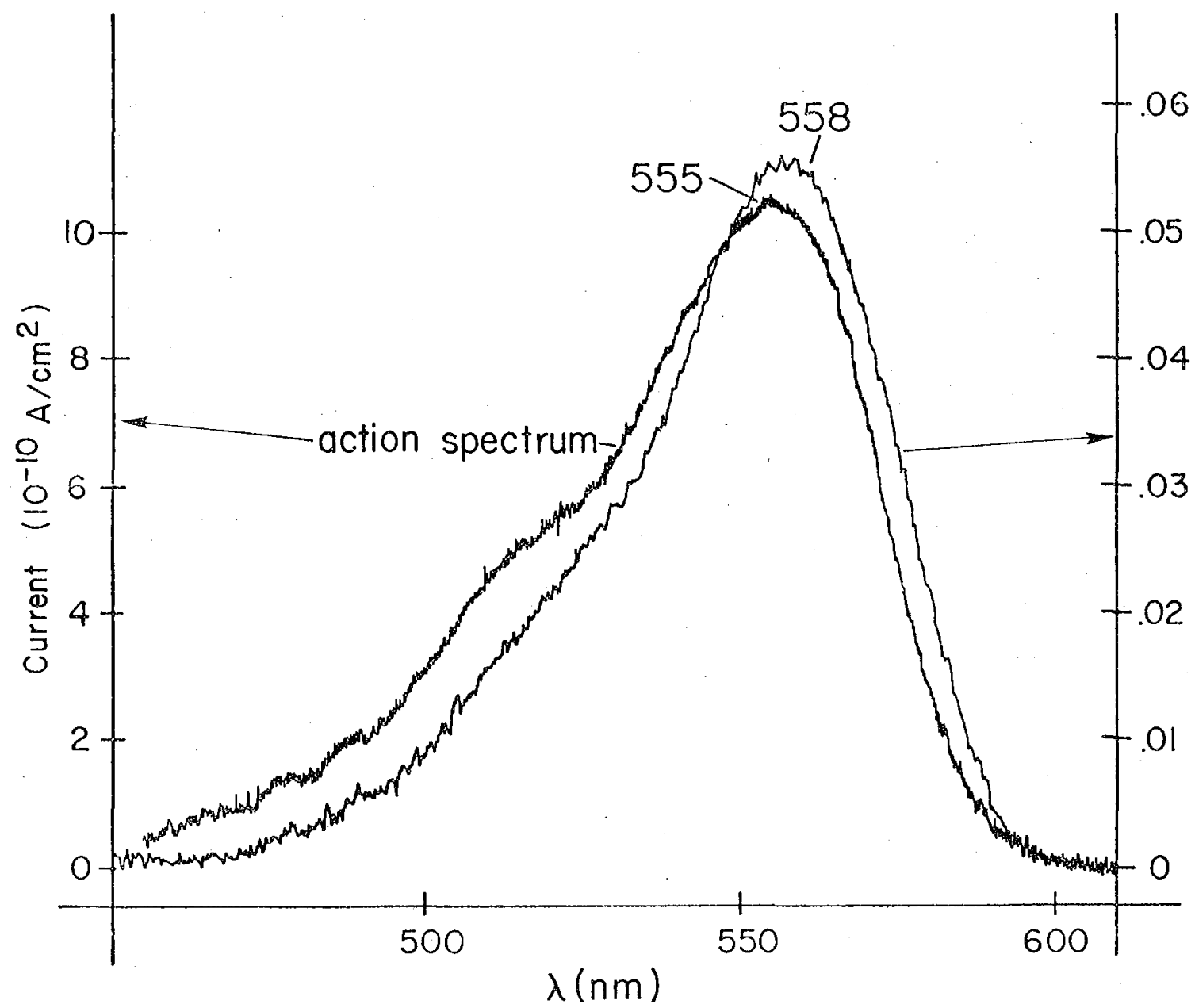

XBL $773-4254$

Figure 29. A comparison. of the photocurrent action spectrum of rhodamine $B$ with the absorbance of the dye adsorbed on $\mathrm{ZnO}$. 
dex of refraction method presented in the discussion of Chapter II since $.25 \mathrm{M} \mathrm{KCl}$ has a higher index of refraction than air. There is also a slightly higher shoulder in the action spectrum where the dimer absorbance of the dye is found. Both these discrepancies are probably due to the presence of the salt in solution. At this dye concentration, it could salt out the dye to form dimers on the surface to some small extent. The Heimholtz layer formed by these ions would also subject the dye to high electric fields of $>10^{5} \mathrm{~V} / \mathrm{cm}$. Dyes exposed to these fields are observed to change their absorbance spectra in many ways of which a spectral shift is one. 61,62 with such minor differences, the action spectrum can be seen as a measure of the absorbance of the adsorbed dye layer.

(ii) Time Decay.

The photocurrent produced, however, does not remain constant with time, but decays rapidly. This decline can be seen in figure 30 when the dye was exposed to monochromatic radiation at the wavelength of maximum response at a $+0.5 \mathrm{~V}$ bias. After five minutes the photoinjection has dropped to $25 \%$ of the initial value as the dye on the surface is oxidized. This decay can be diminished to a great extent through the addition of a reducing agent such as hydroquinone which is known to reduce the dye in its excited state: $7,14,63,64$

$$
\mathrm{R}+\mathrm{D}^{*} \longrightarrow \mathrm{R}^{+}+\mathrm{D}^{-}
$$

The reducing agent can also regenerate the dye from its oxidized form:

$$
\mathrm{R}+\mathrm{D}^{+} \longrightarrow \mathrm{R}^{+}+\mathrm{D}
$$

After a time period where this decay has reached a quasi-steady state, the current voltage curve for this electron transfer can be established. This is shown in figure 31 for a sweep rate of $5.6 \mathrm{mV} / \mathrm{sec}$. The current 


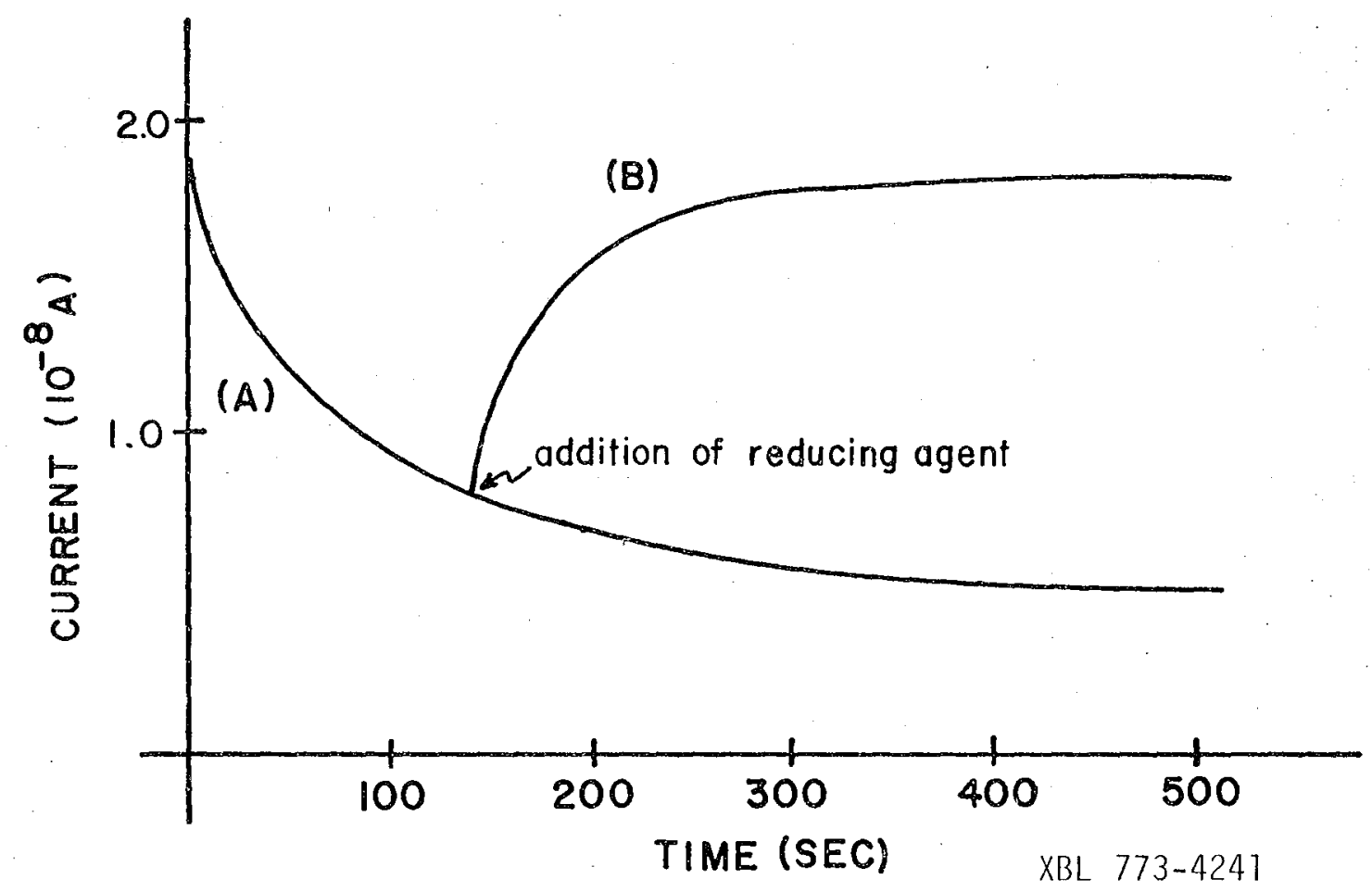

Figure 30. Example of time decay of photocurrent (a). This decay can be halted by addition of reducing agent such as hydrouinone (b). 


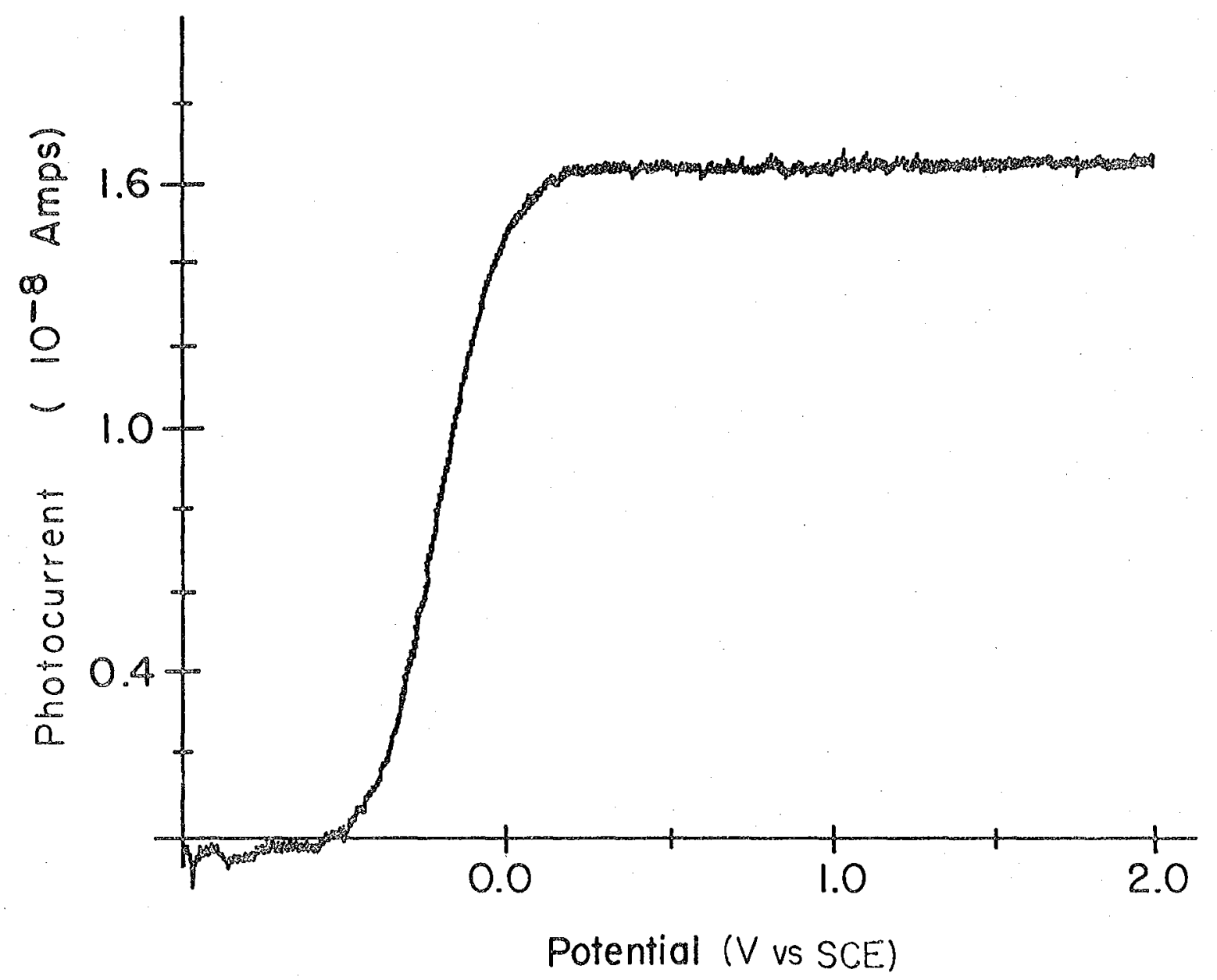

Figure 31. Current voltage behavior of electron transfer. 
rises from zero at $-0.5 \mathrm{~V}$ (SCE) and reaches a saturation by $+0.2 \mathrm{~V}$. Such a relation would also be found for the initial photocurrent except that the value of the saturation limit would be much higher.

A monolayer of rhodamine $\mathrm{B}$ adsorbed on $\mathrm{ZnO}$ was found to correspond to a surface concentration of $6.8 \times 10^{13}$ dye molecules $/ \mathrm{cm}^{2}$. With each dye molecule yielding one electron in the oxidation process, this entire monolayer would be consumed within the first 60 seconds of illumination in the experiment represented by figure 31 . Thus, the oxidized dye molecules must be desorbed and replaced by rhodamine B from solution in order for the current to continue at the observed high consumption rate. This desorption process is probably unnecessary in the presence of reducing agents such as hydroquinone or iodide. In such a situation a dye molecule can be regenerated in situ from an oxidized species (eqn. 13) and a photoreduced dye molecule (eqn. 12) can return to a photoactive state after injection of an electron into the electrode.

The magnitude of the photocurrent observed with $\mathrm{KNO}_{3}$ as an electrolyte differs from that seen with $\mathrm{KCl}$. There has been some work done which suggests that chloride can function as a reducing agent for the excited dye so that it should induce a greater photocurrent than an inert salt such as $\mathrm{KNO}_{3}{ }^{65}$. This is what is observed in figure 32. Further experiments and dsicussion of this photoreductive ability will follow in the next section.

\section{(iii) Photocurrent vs Solution Concentration}

As has already been established, the adsorption of rhodamine $B$ on Zno will vary with solution concentration. Consequently, the photocurrent in an electrochemical arrangement can also be expected to change with the solution concentration of the dye. It should be informative to 


\section{$0000+2+120$}

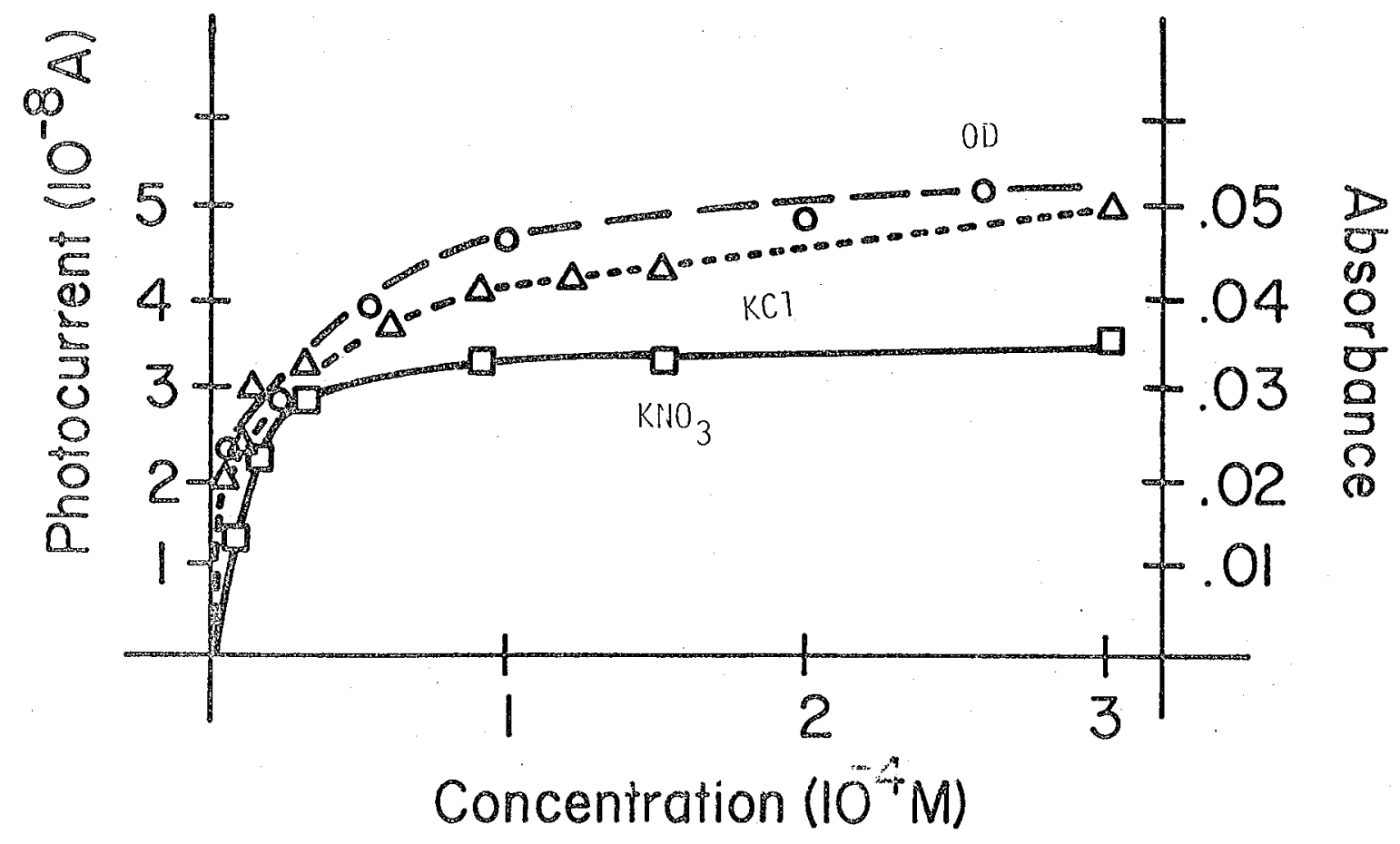

$X B L 773-4248$

Figure 32. The photocurrent from .25 M solutions of $\mathrm{KNO}_{3}$ and $\mathrm{KCl}$ are shown as a function of the dye concentration in solution. The absorbance of the dye layer is also included. 
compare the data from the adsorption isotherm of figure $14 \mathrm{c}$ with data from similar photocurrent measurements to obtain a measure of the quantum efficiency of charge transfer as a function of solution concentration. However, the photocurrent measurement requires the presence of an electrolyte in solution, and this may affect the adsorption of the dye. At $\mathrm{pH} 6.7$ though, rhodamine $B$ is a neutral species so that coulomb forces can be expected to have little influence on adsorption. This has been demonstrated through the measurement of the absorbance of the rhodamine $B$ that was adsorbed onto the $L i$ doped $Z n 0$ crystal from a $3.0 \times 10^{-5} \mathrm{M}$ solution of the dye in the presence of .25 $\mathrm{M}$ solutions of $\mathrm{KNO}_{3}$ and $\mathrm{KCl}$ (see Table 3). Compared with the optical density of the dye adsorbed from water there is little difference in the amount of dye adsorbed on the surface. This confirms literature reports that the adsorption of rhodamine $\mathrm{B}$ is independent of $\mathrm{KCl}$ concentration. 43,65

The magnitude of the photocurrent at the dye's absorbance maximum was determined for solutions up to $3.0 \times 10^{-4} \mathrm{M}$ in rhodamine $\mathrm{B}$ for $.25 \mathrm{M}$. $\mathrm{KNO}_{3}$ and $\mathrm{KCl}$ solutions. The results can be seen in figure 32 along with the optical density measurements from figure $14 \mathrm{~b}$. The three curves are very similar. Each point is the average of six to ten measurements of the photocurrent at its initial value before the time decay. The bias was t0.50 V (SCE). After each measurement the crystal was washed with successive baths of doubly distilled water, methanol, and distilled water. A fifteen minute equilibration time was allowed after immersion of the electrode before illumination and measurement.

As the surface concentration of the dye increases, it is not unreasonable to expect dye-dye interactions such as energy transfer to affect the efficiency of the charge transfer process. This point has been dis- 
Table 3. The adsorption of rhodamine $\mathrm{B}$ on a Li doped $\mathrm{ZnO}$ single crystal does not appear to be affected by the presence of a salt. The accuracy of the figures are limited to $10 \%$.

\begin{tabular}{|c|c|c|c|}
\hline & $\mathrm{KNO}_{3} 25 \mathrm{M}$ & $\begin{array}{c}.25 M \\
\text { KCI }\end{array}$ & $\mathrm{H}_{2} \mathrm{O}$ \\
\hline Absorbance & .0044 & .0045 & .0045 \\
\hline
\end{tabular}

Table 4. The quantum efficiency of electron transfer is listed for $\mathrm{KCl}$ and $\mathrm{KNO}_{3}$ as a function of solution concentration of the dye. It is nearly constant.

$\begin{array}{lll}\begin{array}{l}\text { Solution } \\ \text { Conceptration } \\ \left(\mathrm{x} 10^{5 \mathrm{M})}\right.\end{array} & \mathrm{KNO}_{3} & \mathrm{KCl} \\ 30 & .025 & .037 \\ 15 & .026 & .035 \\ 9 & .026 & .035 \\ 3 & .033 & .036 \\ 1.5 & .031 & .043 \\ 0.75 & .020 & .038 \\ \bar{X} & .027 & .037 \\ \sigma & 17 \% & 8 \%\end{array}$


cussed in previous investigations. 18,19 These interactions could take place via a strong excitonic coupling as occurs in dimers or a weaker long range energy transfer. However, the absorbance spectra of figure 14a exclude all but the weakest of energy transfer interactions.

Directing attention to the size of the photocurrent relative to the absorbance of the adsorbed dye, the data of figure 14 will permit a calculation of the quantum efficiency of charge transfer as a function of surface coverage of the electrode. The results of such a calculation are listed in Table 4 for each surface concentration at which a photocurrent measurement was taken. Complete surface coverage, $\theta=1.0$, is defined as $1.1 \times 10^{-7} \mathrm{mmoles} / \mathrm{cm}^{2}$.

The quantum efficiency of electron transfer appears to be constant with surface coverage although the $\mathrm{KNO}_{3}$ data are not too convincing. The effect of the salt on adsorption may account for the discrepancies as the knee of the $\mathrm{KNO}_{3}$ photocurrent data of figure 32 is different from the knees of the optical density and KCl data. However, the relatively small range of the $\Phi$ data does require serious consideration of the possibility that the efficiency of the charge injection does not depend on the surface concentration of the dye over this range of $\Phi$. This suggests two possibilities. Either the incidence of excitation transfer is negligible or its effect on the electron transfer efficiency has saturated before the coverage of $4.0 \times 10^{-8}$ mmoles $/ \mathrm{cm}^{2}$ has been reached.

For any long range energy transfer between two dye molecules to be efficient, their separation should be no longer than about $50 \stackrel{\circ}{A}$ and their orientations should be compatible. At the lower limit of the surface coverage investigated, the dye is distributed over the surface with an average intermolecular distance of about $20 \AA$. If energy transfer occurs 
in this surface array, it would definitely drop off once the intermolecular separation passed $50 \AA .8$ This corresponds to a surface concentration of about $2 \times 10^{-9} \mathrm{mmoles} / \mathrm{cm}^{2}$ or $\theta=.01$. It is evident that surface concentrations covered in this work are not low enough to decide between these two possibilities.

\section{(b) Time Decay of Photocurrent}

(i) Introduction

As can be seen in figure 30 , the photocurrent produced by rhodamine $B$ at a Zno surface diminishes with time. It is evident that a prolonged exposure of the interface to illumination results in a decrease in the number of photoactive dye molecules at the surface. Gradualiy the surface must become covered with inactive, oxidized rhodamine $B$ which is only slowly replaced with new dye from solution. This picture of the time decay implies that the magnitude of the photocurrent after initial exposure will depend on the relative rates of photooxidation of the dye and replacement of the oxidized molecule.

An attempt will be made to correlate this intuitive picture of the time decay with the observed decline in the photocurrent. Two sets of data are available for examination. One is the time behavior of the photocurrent for rhodamine $\mathrm{B}$ in an inert electrolyte, $0.25 \mathrm{M} \mathrm{KNO}_{3}$, and the other is the corresponding decay for the dye in $1.0 \mathrm{M} \mathrm{KCl}$ which functions to a small extent as a reducing agent.

A model for this decay was formulated using classical kinetics with the assumption that there are two distinct types of adsorbed rhodamine $B$, one of which photoinjects electrons at a more efficient rate than the other. Analysis of the data with this assumption yields the relative quantum efficiencies of these two differently adsorbed dye species. In- 
formation concerning desorption rates of the oxidized dye and the total surface concentration of the dye can also be obtained.

\section{(ii) Resuits}

Two different electrolytes were used in an investigation of the time behavior of the photocurrent, $1.0 \mathrm{M} \mathrm{KCl}$ and $0.25 \mathrm{M} \mathrm{KNO}_{3}$. Both of these solutions were $1.5 \times 10^{-4} \mathrm{M}$ in rhodamine $\mathrm{B}$, a concentration corresponding to the plateau region of the adsorption isotherm of figure 14b. Qualitatively, the results were similar although the magnitude of the photocurrent from the $\mathrm{KCl}$ experiments was larger than the $\mathrm{KNO}_{3}$ results. The data includes results from both electrolytes. The basic photoelectrochemical set-up remains the same. The photocurrent always represents that obtained at a saturation bias; monochromatic illumination through the crystal as an optical window at the dye's absorbance maximum was used. The solutions were deoxygenated and stirred with ultrapure $\mathrm{N}_{2}$. As can be seen in figure 33, the photocurrent decays in time with exponential characteristics. This Togarithmic analysis of the time decay reveals four time constants. Two are small and their effect disappears within the first few hundred seconds; the remaining two represent long term decays in the thousands of seconds.

At this point it is appropriate to mention that accompanying this photocurrent decay is a dc dark current that results from the potentiostatic control of the electrode's bias potential. As a polarizable semiconductor electrode, zinc oxide is nearly ideal with dark currents of about 5 nanoamps in these experiments at $\mathrm{pH} 6.5$. This current is thought to be partially ionic in character and has been attributed to two sources: 66 the anodic dissolution of the Zno lattice into $\mathrm{Zn+t}$ ions and $\mathrm{O}_{2}$ gas, and the ionic migration of interstitial $\mathrm{Zn}^{+}$to the surface where it is oxi- 

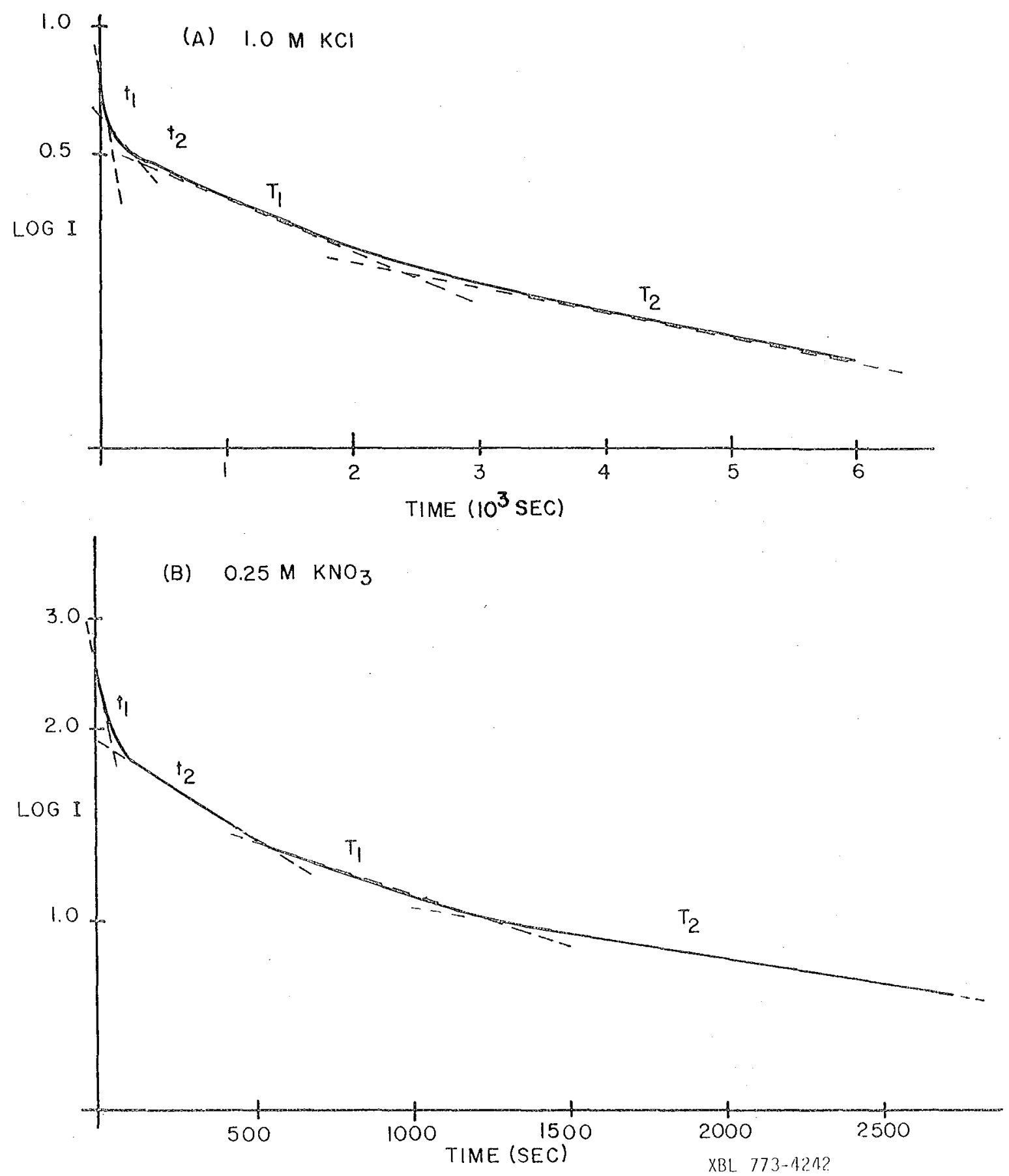

Figure 33. A logarithmic plot of the decay of the photocurrent reveals four time constants.

(a) $1.0 \mathrm{M} \mathrm{KCl}$ as electrolyte

(b) $0.25 \mathrm{M} \mathrm{KNO}_{3}$ as electrolyte 
dized and solvated. The dark current decays exponentially with time; physically, it can be pictured as an electrical discharge of the surface as surface charges with energies above the maintained electrochemical potential are drained from the interface with an RC time constant characteristic of the surface capacitance and the exchange current of the specific surface species involved.

Several experiments serve to clarify the role of this potentiostatic dark current in the long term decay of the dye sensitized photocurrent; two of these experiments are shown in figure 34 . In figure $34 a$ the photocurrent was measured as a function of time, but with significant periods during which the crystal was in the dark. It can be seen that the long term decay is dependent on the dark current; if the experiment is run with an interruption of the light and the potentiostatic control then the long term decay in the dark is halted (figure $34 \mathrm{~b}$ ). These results indicate that the large decay constants originate from processes closely tied to the dark current.

In figure 35 can be seen an extension of the experiments depicted in figure $34 \mathrm{a}$. Here the dependence of both long term decays on the dark current is easily seen. Accompanying each exposure to the light is a recurrence of the two fast time decays; a log plot of these fast decays for the first three exposures is shown in figure 36 . It is evident that with each successive exposure, the first time constant plays a smaller role in the fast decay. It appears to be connected with the first long time constant $T_{1}$ and disappears by the time that the second long time constant determines the reaction rate.

These time constants were not quantitatively reproducible. The photooxidation of the dye is expected to be very sensitive to surface details and these will change with long periods of anodic polarization. A 5 nan- 
$0004 \% 0124$

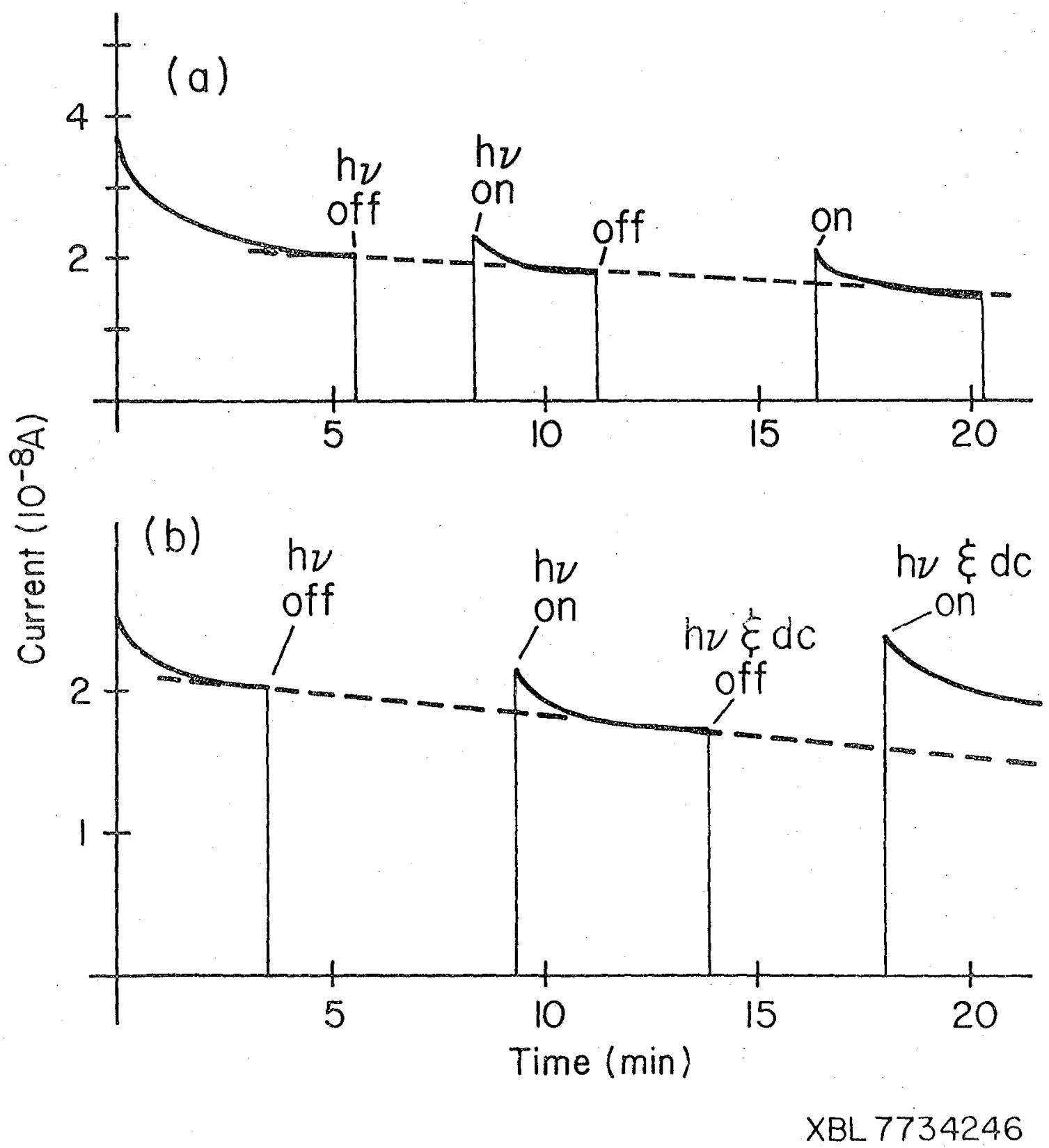

Figure 34. (a) Potentiostatic control of the crystal results in a long term decay of the dark current even when the interface is not exposed to light. (b) Removal of potentiostatic control allows a recovery of the photocurrent and halts the long term decay. 

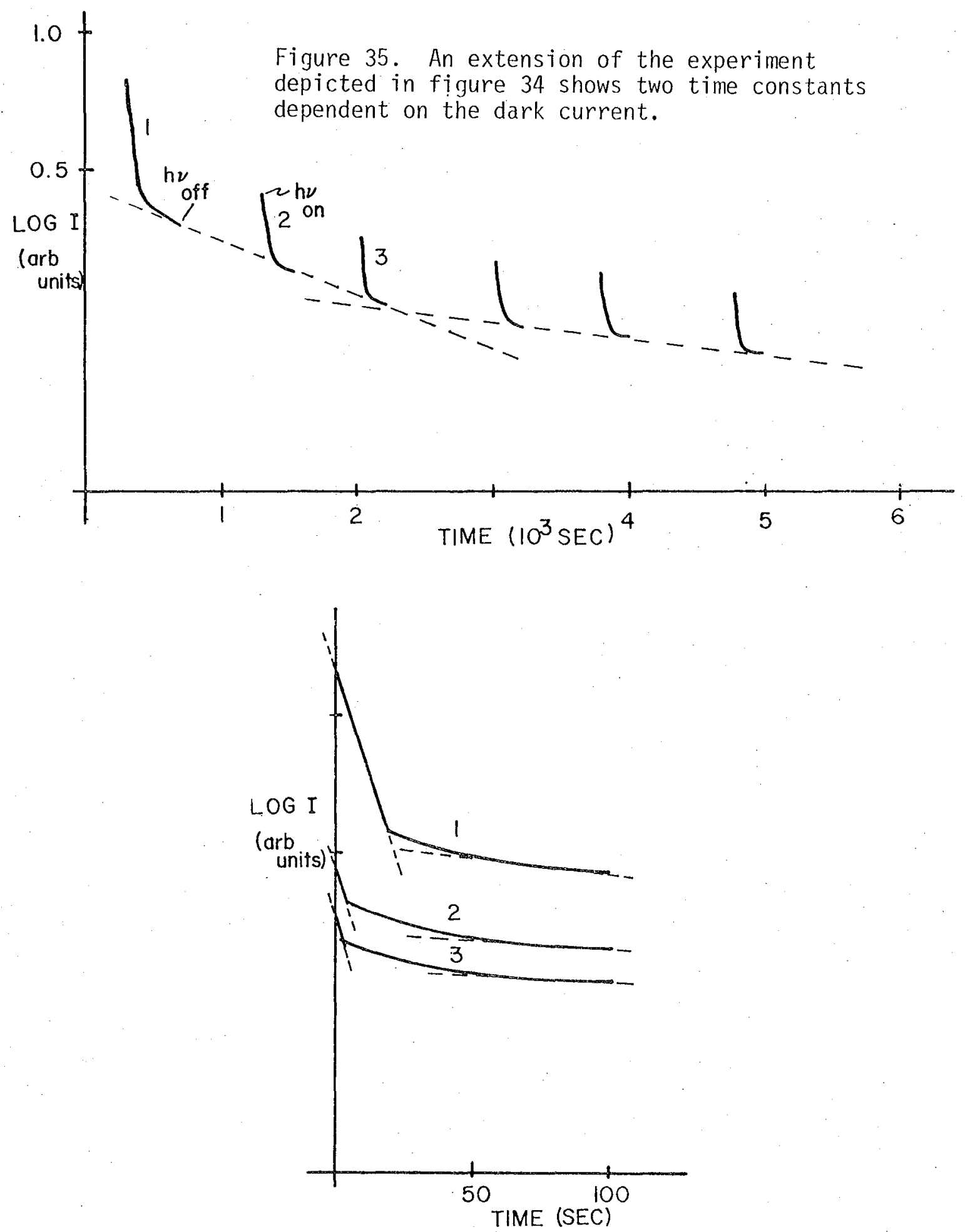

XBL $773-4243$

Figure 36. A magnification of the first three exposures of figure 35 shows the decreasing role of $t_{1}$ with time. 


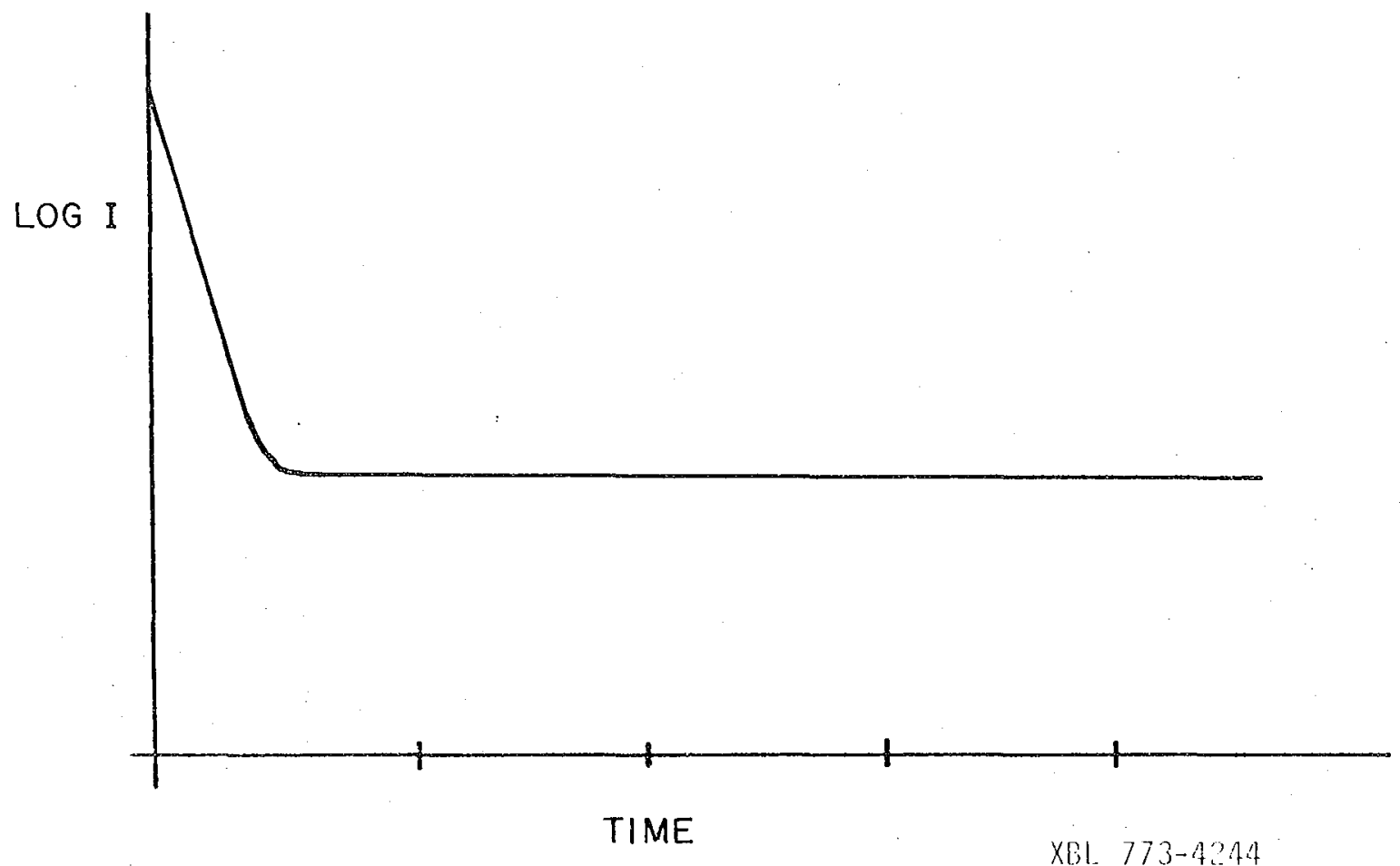

Figure 37. Sample plot of photocurrent decay equation without long term decay (eqn (16)). 
oamp dark current should lead to dissolution of $50 \%$ of the surface after 2000 seconds of polarization.

Furthermore, the dye's adsorption and desorption rates are dependent on the stirring of the electrolyte. With the experimental apparatus used in these experiments, it was not possible to reproduce this stirring rate from experiment to experiment. 
A correlation of the sensitized decay constants with the dark current was not possible because of problems isolating the metalsemiconductor contact from the electrolyte. The resulting extraneous currents produced at this metal-electrolyte could not be eliminated and were, in general, equal to, or greater than, the real dark current through the semiconductor. The 5 nanoamp figure quoted here was the smallest measured dark current.

\section{(iii) Derivation of Kinteic Mode]}

An analysis of the kinetics of this time decay in the absence of reducing agents requires consideration of the following processes at the electrode surface:

$$
\begin{aligned}
& \mathrm{D}_{\mathrm{SO}}+\mathrm{S} \stackrel{\mathrm{Ki}}{\longrightarrow} \mathrm{D} \quad \text { adsorption of dye on site " } \mathrm{s} \text { " } \\
& 0+h \nu \stackrel{\alpha \Omega}{\rightarrow} D^{*} \quad \text { excitation of adsorbed dye } \\
& D^{*} \quad k_{2} \rightarrow D^{+}+e^{-} \quad \text { oxidation of adsorbed dye } \\
& D^{+} \quad \mathrm{k}_{3} \rightarrow \mathrm{D}_{\mathrm{SO}}^{+} \quad \text { desorption of oxidized dye } \\
& D^{*} \quad \mathrm{k}_{4} \rightarrow \mathrm{D} \quad \text { excitation relaxation processes }
\end{aligned}
$$

Here the molar extinction of the dye, $\varepsilon$, is represented as a molecular cross section $\alpha$ with units of $\mathrm{cm}^{2} /$ molecule. The photon flux is represented by $\Omega$.

The photocurrent measured at an interface reflects the oxidation of the excited dye

$$
J=k_{2}\left[D^{*}\right]
$$

The size of $\left[D^{*}\right]$ can be determined through solution of this differential equation:

$$
\frac{d\left[D^{*}\right]}{d t}=\alpha \Omega[D]-\left(K_{4}+K_{2}\right)\left[D^{*}\right]
$$


The solution to this equation in a steady state approximation is:

$$
\left[D^{*}\right]=\frac{\alpha \Omega[D]}{k_{2}+k_{4}}
$$

In order for the time dependence of [D] to be established, the adsorption processes described by rate constants $k_{7}$ and $k_{4}$ must be considered. At the solution concentrations of rhodamine $B$ used in these experiments, $1.5 \times 10^{-4} \mathrm{M}$, it can be assumed that the steady state concentration of vacant surface sites "s" is a constant. This implies that every site, s, vacated by desorption of $D^{+}$is filled by an unoxidized D from solution. With this steady state assumption for [s], an expression describing the time dependence of [D] may be written:

$$
\frac{d[D]}{d t}=-\alpha \Omega[D]+K_{3}\left[D^{+}\right]+K_{4}\left[D^{*}\right]
$$

With the use of eqn. 14 and a mass balance expression,

$$
\left[D_{0}\right]=\left[D^{+}\right]+[D]+[D *]
$$

where $\left[D_{0}\right]$ is the total number of rhodamine $B$ molecules on the surface, eqn. 15 may be written

$$
\frac{d[D]}{d t}=-\alpha \Omega\left(\frac{k_{2}+K_{3}}{K_{2}+K_{4}}\right)[D]-k_{3}[D]+k_{3}+\left[D_{0}\right]
$$

The solution to this equation is

$$
[0]=\frac{\left[D_{0}\right]}{k_{3}+Q}\left\{k_{3}+Q e^{-\left(Q+k_{3}\right)} \cdot t\right\}
$$

where

$$
Q=\alpha \Omega\left(\frac{k_{2}+k_{3}}{k_{2}+k_{4}}\right)
$$

Substituting this into eqn. 14 yields an expression for the phorocurrent

$$
J=\left(\frac{k_{2}}{k_{2}+k_{4}}\right) \alpha \Omega \frac{\left[D_{Q}\right]}{k_{3}+Q}\left\{k_{3}+Q_{e}-\left(Q+k_{3}\right) t\right\}
$$

where $\Phi=\left(\frac{k_{2}}{k_{2}+k_{4}}\right)$ is the quantum efficiency of the photoinjection process. 
A plot of eqn. 16 yields a curve which does not agree with the observed experimental time decay. This simple model assumes that after the initial sharp decay, the concentration of dye at the surface will depend on the rate constant $k_{3}$ for desorption of $D^{+}$. This will be a constant determined by the stirring rate of the solution; the photocurrent should then remain constant as depicted in figure 37.

However, it has been observed (figure 34a) that continuous potentiostatic control of the crystal results in long term time constants for decay that apply even in the dark. This can be explained qualitatively as a decrease in the number of surface sites available for occupation by photoactive molecules. In the mass balance expression, $D_{0}$, the number of sites available for occupation, should not be constant but will decay exponentially with a rate constant $k_{6}$ :

$$
\left[D_{t}\right]=\left[D_{0}\right] e^{-k_{6} t}
$$

Such a blockage of the surface may proceed through several processes. The dark current production of oxygen at the crystal surface may result in adsorption of molecular oxygen and subsequent abstraction of an electron to form $\mathrm{O}_{2}^{-}$at the electrode. The $\mathrm{O}_{2}$ at the surface could also react with the adsorbed dyestuff to form non-desorbable oxidation products. Both of these reactions would result in a physical occupation of the surface by photoinactive species other than the oxidized dye molecule.

The introduction of eqn. 17 into eqn. 15 gives a new solution for the photocurrent decay.

$$
J=\left(\frac{k_{2}}{k_{2}+k_{4}}\right) \alpha \Omega-\frac{\left.1 D_{0}\right] e^{-k_{6} t}}{\left(k_{6}+k_{3}+Q\right)}\left\{k_{3}^{\prime}+\left(Q+k_{6}\right) e^{-\left(\dot{Q}+k_{3}-k_{6}\right) t_{3}}\right.
$$


This model for the time decay accounts for two time constants. One is large, $\left(k_{6}+Q+k_{3}\right)$, and the other is small, $k_{6}$.

These constants agree with the data in figure 36 in that a fast decay is coupled with a slower one; after the initial dye layer is quickly oxidized, the rate limiting step is its replacement by intact solution species. This replacement gradually decreases as the number of photoactive surface sites is diminished through an undetermined dark reaction.

The observation that there are two such couples in the experimental decay curve suggests that every dye molecule adsorbed onto the surface does not exhibit the same quantum efficiency of sensitization, $\Phi$, but, instead, falls into one of two groups. The differing ability of adsorbed rhodamine $B$ molecules to sensitize $\mathrm{ZnO}$ may be due to different adsorption orientation or different adsorption sites. These would also affect the desorption rate of an oxidized dye molecule.

Consequently, in the interpretation of the data of figure 33a according to eqn. 18, it will be assumed that there are two kinds of adsorbed dye with two different sensitizing abilities. Thus, the total observed photocurrent will be the sum of contributions from these two sources, each with its own set of decay time constants:

$$
\mathrm{J}_{\text {tot }}=\mathrm{J}_{1}+\mathrm{J}_{2}
$$

This may be written in full for both as:

$$
J_{\text {tot }}=\alpha_{1} e^{-\gamma_{1} t}+\beta_{1} e^{-\delta_{1} t}+\alpha_{2} e^{-\gamma_{2} t}+\beta_{2} e^{-\delta_{2} t}
$$

where

$$
\begin{aligned}
& \alpha_{i}=\Phi_{i} \alpha \Omega\left(\frac{k_{3}}{k_{\sigma_{1}}+k_{3}+Q}\right)\left[D_{0}\right]_{i} \\
& \beta_{i}=\Phi_{i} \alpha \Omega\left(\frac{k_{6}+{ }^{2}}{k_{6}+k_{3}+Q}\right)\left[D_{0}\right]_{i} \\
& \gamma_{i}=\left(k_{6}+k_{3}+Q\right)
\end{aligned}
$$




$$
\delta_{i}=k_{6 i}
$$

An analysis of the photocurrent decay of figure 33 for rhodamine $B$ adsorbed from a $\mathrm{KNO}_{3}$ electrolyte yields values for the constants of eqn. 19. They are listed in Table 5. The arithmetical manipulations required for extraction of these values from figure 33 are included in Appendix I .

With $\alpha, \beta, \gamma$, and $\delta$ known, the constants $k_{6}, k_{3}$ and $Q$ can be determined for each " $i$ " from eqns. 20 through 23. Additionally, if it is assumed that $k_{3} \ll k_{2}$, i.e., the rate constant for desotpion of the oxidized dye molecule is much smaller than that for electron transfer, then

$$
Q=\left(\frac{k_{3}+k_{2}}{k_{2}+k_{4}}\right) \alpha \Omega \sim \Phi \alpha \Omega
$$

With this assumption, the concentration of each class $\left[D_{0}\right]_{i}$ of dye molecules on the surface can be determined. This information permits calculation of the $\left[D_{0}\right]_{i}$ for the two classes since the overall quantum efficiency at $t=0$ is known to be .033 . All of these results are listed in Table 5.

An estimate of the molecular cross section $\alpha$ can be made since the photon flux $\Omega$ is a known quantity. This can be compared with the measured value of $\alpha$ for rhodamine $B^{10}$ which is also listed in Table 5 .

An analysis of the time decay when the electrolyte contains a reducing agent would be more complex than the simple model presented here. The complexity results from the competition between the two energetically plausible reactions of a reducing agent:

$$
\begin{array}{ll}
R+D^{*} & \longrightarrow D^{-}+R^{+} \\
R+D^{+} \longrightarrow D^{+} & \longrightarrow R^{+}
\end{array}
$$

If one reaction could be excluded, a relatively simple analysis could 
Table 5. The data from the kinetic analysis

\begin{tabular}{|c|c|c|c|}
\hline$i$ & 1 & $\underline{2}$ & units \\
\hline$\alpha_{i}$ & $3.96 \times 10^{11}$ & $2.36 \times 10^{11}$ & $\mathrm{sec}^{-1} \mathrm{~cm}^{-2}$ \\
\hline$\beta_{j}$ & $2.56 \times 10^{11}$ & $9.11 \times 10^{11}$ & $\mathrm{sec}^{-1} \mathrm{~cm}^{-2}$ \\
\hline$\gamma_{j}$ & $9.95 \times 10^{-3}$ & $1.04 \times 10^{-3}$ & $\sec ^{-1}$ \\
\hline$\delta_{i}$ & $4.68 \times 10^{-4}$ & $7.98 \times 10^{-5}$ & $\sec ^{-1}$ \\
\hline$Q_{i}$ & $3.43 \times 10^{-3}$ & $7.49 \times 10^{-4}$ & $\sec ^{-1}$ \\
\hline$k_{3 i}$ & $6.04 \times 10^{-3}$ & $2.14 \times 10^{-4}$ & $\mathrm{sec}^{-1} \mathrm{~cm}^{-2}$ \\
\hline$K_{6 i}$ & $4.68 \times 10^{-4}$ & $7.98 \times 10^{-5}$ & $\mathrm{sec}^{-1} \mathrm{~cm}^{-2}$ \\
\hline$[D o]_{j}$ & $1.90 \times 10^{74}$ & $1.53 \times 1015$ & molecules $/ \mathrm{cm}^{2}$ \\
\hline$\Phi \dot{j}$ & .115 & .025 & {$[-$} \\
\hline$\alpha_{j}$ & $3.2 \times 10^{-17}$ & {[} & $\mathrm{cm}^{2} /$ molecule \\
\hline$\Omega_{j}$ & $1.85 \times 10^{15}$ & & Photons $/ \mathrm{cm}^{2}-\mathrm{sec}$ \\
\hline$\alpha_{i}$ & $3 \times 10^{-16}$ & 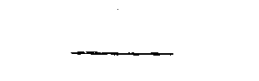 & $\mathrm{cm}^{2} /$ molecule \\
\hline
\end{tabular}


be formulated for the other. However, until some measure of the relative values of $k_{A}$ and $k_{B}$ has been made, such an analysis would be speculative and has not been attempted here.

Furthermore, a much more rigorous control of experimental conditions would be required in an examination of this decay. Specifically, a rotating electrode would be necessary for accurate and complete control of the diffusion processes at the surface. (iv) Discussion

This interpretation of the photocurrent time decay has been based on the assumption that the dye adsorbed on the electrode is photooxidized at two different rates. These rates are reflected in the two different: quantum efficiencies, $\Phi_{7}=11.5 \%$ and $\Phi_{2}=2.5 \%$ that were derived for this system. According to the definition $\Phi=k_{2} /\left(k_{2}+k_{4}\right)$ the difference between these two efficiencies can be seen to originate in the relative rates of electron transfer, $k_{2}$, and decay from the excited state, $k_{4}$. Dye adsorption at the different surface sites must then affect the relative values of these competing processes.

The assumption that there are two distinctly different relationships 
between $k_{2}$ and $k_{4}$ for the adsorbed dye molecules is interesting. Considering the weak adsorption of the dye to the surface, it is unlikely that $k_{4}$ will change from site to site. It is probable then, that the electron transfer rate $k_{2}$ is the significant factor contributing to these differing efficiencies.

The $k_{6}$ values in Table 5 also imply that the more efficient adsorption sites are disabled faster through the dark reaction than the less efficient sensitizing sites even though the desorption rate of oxidized molecule is faster from this site. With fewer adsorption sites, a greater rate of disablement would insure a relatively quick disappearance of sensitization from rhodamine $B$ adsorbed on these locations.

This analysis of the time decay of figure 33b does not yield quantitatively reliable information. Unfortunately, the experimental conditions are much more complex than the simple model presented here because of the modulation techniques employed for current detection. In the chopping cycle each exposure of the surface to light is followed by a dark period during which the system can recover to some extent. Consequently, the observed decay is not as fast as it would be under a condition of continuous illumination.

As a result, some of the data tabulated in Table 5 are clearly in error. The total surface concentration of the dye is at least an order of magnitude too large while the calculated $\alpha$ is only a tenth of its measured value. Both of these values are derived from $Q$ which is determined by the time decay of the photocurrent. Therefore, they cannot be expected to be correct. 
(c) Photoreduction of Rhodamine B at the Zn0 Electrode

(i) Introduction

It was seen in figure 32 that the quantum efficiency of electron transfer in a system with a $\mathrm{KCl}$ electrolyte exceeds that observed with a $\mathrm{KNO}_{3}$ electrolyte. The difference in photocurrent for these two systems has been reported in the 1 iterature, ${ }^{43,65}$ but no measure of the quantum efficiency of these reactions has been possible heretofore because the optical density of the dye layer was not known. In this section the quantum efficiency of electron transfer from excited rhodamine $B$ will be determined for electrolytes which contain $\mathrm{Cl}^{-}, \mathrm{Br}^{-}, \mathrm{I}^{-}$, and hydroquinone.

The photoreduction of excited dyes on the surface of semiconductors leading to increased electron injection into the electrode has been termed supersensitization. ${ }^{67}$ Tributsch and Gerischer ${ }^{14}$ have propsed that this reaction proceeds through adduct formation between the excited dye and reducing agents such as hydroquinone and allyl thiourea. This leads to the reduction of the dye and subsequent transfer of an electron to the electrode:

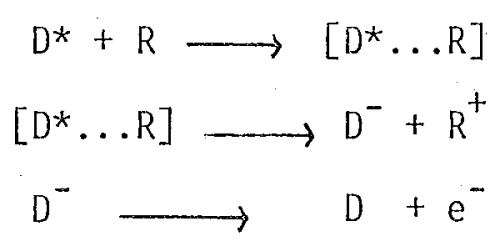

This adduct proposal is supported by the fluorescence quenching of dyes by these reducing agents. $7,16,63,64$ It has also been reported that the halides quench the fluorescence of rhodamine $B$.

The quantum efficiency for photoreduction of adsorbed rhodamine B for these reducing agents can be determined electrochemically by the measured increase in photocurrent induced by reaction 24 . Such a measurement has been made and will be presented here. 


\section{(ii) Experimental}

Six solutions of $3.0 \times 10^{-5} \mathrm{M}$ rhodamine $\mathrm{B}$ were prepared with one solution each of $0.25 \mathrm{M} \mathrm{KI}, \mathrm{KBr}, \mathrm{KNO}_{3}, \mathrm{~K}_{2} \mathrm{SO}_{4}$, and one with $0.25 \mathrm{M} \mathrm{K}_{2} \mathrm{SO}_{4}$ and $2.5 \times 10^{-3} \mathrm{M}$ hydroquinone. The adosrption of rhodamine $\mathrm{B}$ from these solutions onto the $\mathrm{Li}$ doped $\mathrm{ZnO}$ crystal was established through absorbance measurements. The results are listed in Table 6. All solutions were adjsuted to $\mathrm{pH} 6.7$. The $\mathrm{ZnO}$ electrode was biased at $0.50 \mathrm{~V}$ (SCE).

Photocurrent measurements were recorded for these solutions and are also listed in Table 6 . This permits a calculation of the relative quantum efficiency for electron transfer assuming that the relative ability of the dye to adsorb from these solutions on the Li doped Zno will also apply for the undoped $\mathrm{ZnO}$ used as the electrode. If it is recalled from Table 4 that $\Phi$ for $\mathrm{KNO}_{3}$ is .033 then the remainder of the quantum efficiencies can be calculated from this one value.

\section{(iii) Discussion}

These data do not agree with the photoelectrochemical data reported by Bode, et a1. ${ }^{65}$ for rhodamine $B$ at $\mathrm{ZnO}$ electrodes immersed in an electrolyte buffered at pH 4.6. Assuming no change in the dye's surface concentration, the relative quantum efficiencies of photooxidation reported from this source for this salt concentration and these ions would be those listed in column 5 of Table 6 .

The discrepancies between the data of Bode et al. and those found here can be attributed to the pH of the electrolyte. At the pH of these experiments, 6.7, rhodamine is a neutral species while at $\mathrm{pH} 4.6$ it is cationic. This would lead to a definite change in the attraction of the dye for a negative reducing species such as the halides.

In fact, in the case of iodide, a charge transfer complex is visible 
in acidic solutions. This can be seen in figure 38 as a change in the absorbance of the dye as the $\mathrm{pH}$ decreases. A new transition is evident at $610 \mathrm{~nm}$ which increases at lower $\mathrm{pH}$. The total absorbance is less because reduction of the dye involves abstraction of a proton and precipitation from solution. Consequently, it should be expected that the reduction of the dye should increase dramatically at low pH. Although a charge transfer complex was not found for $\mathrm{Br}^{-}$and $\mathrm{Cl}^{-}$, it must be concluded that coulomb forces are significant in the interaction between the excited rhodamine $B$ and these ions, too.

From these values of $\Phi$ listed in Table 6 , an overall rate constant $k_{0}$ can be derived for the reaction of these reducing agents with excited dye molecules on the surface. To obtain $K_{0}$ for these species, it will be assumed that all photoreduced molecules on the surface are oxidized to produce the current by which this photoreduction rate is measured. This one to one relation between photoinjection and photoreduction has been confirmed experimentally with hydroquinone and rhodamine $B$ at a cas electrode. 7

In the case of adduct formation, the tendency of a reducing agent to associate with an excited dye molecule may be described by rate constants $k_{a}$ and $k_{a}-$.

$$
D^{*}+R \underset{k_{a^{-}}}{\stackrel{k_{a}}{\leftarrow}}\left[D^{*} \ldots R\right]
$$

The reduction of the dye will proceed from this adduct in competition with a nonreactive decomposition:

$$
\begin{array}{ll}
{\left[D^{*} \ldots R\right] \stackrel{k_{r}}{\longrightarrow}} & D^{-}+D^{+} \\
{[D * \ldots R] \stackrel{k_{d}}{\longrightarrow}} & D+R
\end{array}
$$

Using a steady state assumption for the concentration of the adduct, the 


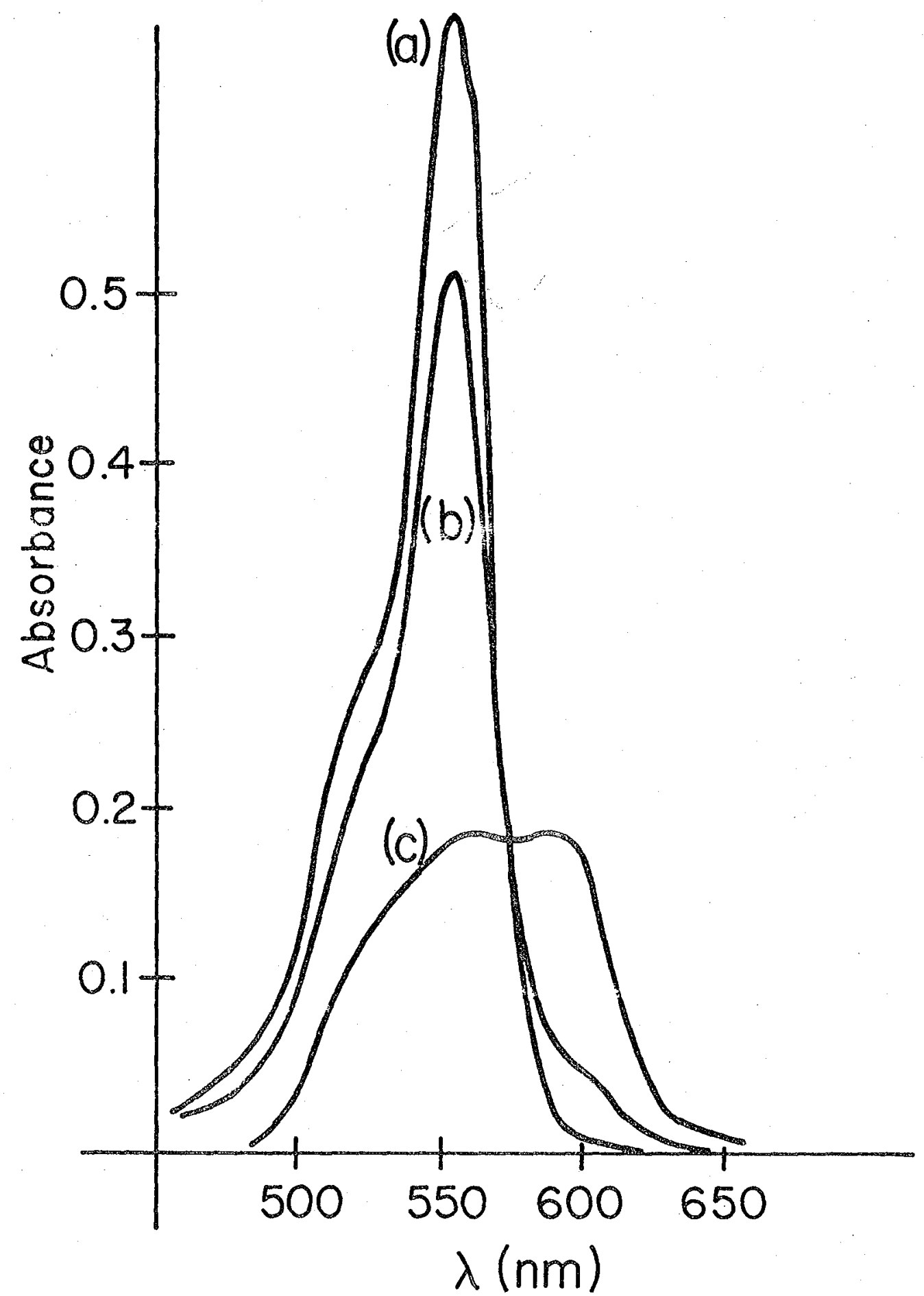

Figure 38. These spectra represent rhodamine $B$ at about $3 \times 10^{-5} \mathrm{M}$ in the presence of .25 $M \mathrm{KI}$ at three different $\mathrm{pH}^{\prime} \mathrm{s}$. At low pH a charge transfer band at $610 \mathrm{~nm}$ emerges.

(a) $\mathrm{pH}$ (a) 8.3 (b) 6.0 (c) 3.9

$773-4251$ 


\section{$00+047+0132$}

overall rate constant $k_{0}$ can be derived:

$$
k_{0}=\frac{k_{r} \cdot k_{a}}{k_{r}+k_{d}+k_{a}-}
$$

In the absence of a reducing agent, the quantum efficiency of electron transfer is: $\Phi_{0}=k_{2} /\left(k_{2}+k_{4}\right)$. The data of Table 4 give a value for $\Phi$ of .033 . Thus, $k_{2}<<k_{4}$. An estimate for $k_{4}$ can be taken from the singlet lifetime, $\tau$, of the excited dye in solution which is about $3 \times 10^{-9} \mathrm{sec}$. Although the lifetime of the excited, adsorbed dye may be much shorter, this should serve as an upper limit for $\tau$. Therefore, a lower limit on $k_{2}$ can be seen as:

$$
k_{2}=\Phi_{0} / \tau=1.1 \times 10^{7} \mathrm{M}^{-1} \mathrm{sec}^{-1}
$$

In the presence of a reducing agent the quantum efficiency of electron injection into the conduction band is

$$
\Phi_{R}=\frac{k_{2}+k_{0}[R]}{k_{2}+k_{4}+k_{0}[R]}
$$

if it is assumed that $k_{r}>>k_{d}$. Assuming that $\left(k_{2}+k_{0}[R]\right)<<k_{4}$, this expression may be rewritten:

$$
\Phi_{R}=k_{2} \tau+k_{0}[R] \tau=\Phi_{0}+\Delta \Phi
$$

In this case, the magnitude of $k_{0}$ is:

$$
k_{0}=(\Delta \Phi /[R] \cdot \tau)
$$

However, given the measured values of $\Phi$ in Table $6, k_{0}$ is not insignificant in comparison with $k_{4}$. In the case of $I^{-}$the ratio $\left(k_{0} / k_{4}\right)$ is about .15. Consequently an iterative procedure using eqns. 26 and 27 was used to establish the $k_{0}$ figures in Table 6 for the various reducing agents used in these experiments.

The solution concentration of hydroquinone was 2.5 millimolar. However, the data in Table 6 indicate that the adsorption of the dye is 
affected through competitive adsorption by hydroquinone. Thus, the local surface concentration may be much higher than that in solution. From the difference in absorbance between the dye adsorbed from the $\mathrm{k}_{2} \mathrm{SO}_{4}$ solutions with and without hydroquinone was made. An upper limit to the volume occupied by the molecule would be that occupied by rhodamine B, about $600 \mathrm{~A}^{3}$. With this figure and the data from Table 6 , the local concentration of hydroquinone was found to be $0.3 \mathrm{M}$. The overall rate constant $k_{0}$ for hydroquinone is also included in Table 6.

The desired information is the value of $k_{r}$ for photoreduction of excited rhodamine $\mathrm{B}$ at the $\mathrm{ZnO}$ surface:

$$
\left[R \ldots D^{*}\right] \stackrel{k_{r}}{\longrightarrow} D^{-}+R^{+}
$$

As was shown here, it is not possible to obtain this rate constant from electrochemical measurements. Only the overall rate constant $k_{0}$ can be established. In order to extract values for $k_{r}$ for each of the reducing agents, kinetic studies of the fluorescence quenching of rhodamine $B$ in solution would have to be made at the same conditions used for these electrochemical measurements. In this way the unknowns $k_{a}, k_{a}-$, and $k_{d}$ in eqn. 25 could be determined.

However, the electrochemical data in Table 6 do provide information concerning the most efficacious reducing agent. Even though hydroquinone has a much more negative redox potential than $I^{-}$(-.12 vs SHE compared with +54$)$, it does not induce greater photocurrents than $\mathrm{I}^{-}$. This may result from larger values of $k_{a}$ - and $k_{d}$ in eqn. 25 , but it is also possible that $k_{r}$ is smaller. 
IV. ELECTRON TRANSFER AT ROSE BENGAL SENSITIZED $\mathrm{TiO}_{2}$ ELECTRODES

A. Introduction

After this initial introduction to experimentation in dye sensitization with rhodamine $B$ and $\mathrm{ZnO}$, it was decided to extend these investigations to other semiconductor substrates. One compound which had become noteworthy ${ }^{69}$ because of its electrochemical properties was $\mathrm{TiO}_{2}$. At that time little work had been reported on the dye sensitization of this semiconductor, and as it was readily available in the laboratory, work was started. using rose bengal as the sensitizing agent.

The goal was to pursue the investigation of this system in the same manner as with the rhodamine $B$ and ZnO so that a quantitative description could be obtained. Towards this end adsorption isotherms were established for this dye on the crystals to be used as electrodes. The combined surface area of a several crystals was large enough to measure this adsorption empirically through elution of the dye without resorting to the theoretical techniques used in Chapter II. Since the crystal is inert to electrochenical dissolution, 70,71 this adsorption and absorbance information would still be applicable when these same crystals were employed as electiodes.

In order to probe the energy level relationships between the first excited state of rose bengal and the $\mathrm{TiO}_{2}$ conduction band, the $\mathrm{pH}$ dependence of the photooxidation rate was measured. In coordination with these measurements a temperature studv was made so that an Arrhenius activation energy for electron transfer could be derived. 72,75 
An unexpected development in the work with this system was the observation of a photoreduction reaction at the electrode surface whereby an electron leaves the conduction band to reduce the oxidized dye molecule in solution.

\section{B. Experimental}

Rose bengal was obtained commercially and purified through recrystallization in ethanol. In these experiments all dye solutions were made with distilled water and were $1 \mathrm{M}$ in $\mathrm{KCl}$. In this solution the dye accepted an electron from a mercury electrode at a potential of -1.08 V vs. a saturated calomel electrode (SCE).

The $\mathrm{TiO}_{2}$ was purchased from Nakazumi Crystals of Osaka, Japan. The crystal came as a long boule grown along the " $c$ " axis by flame fusion. Slices $250 \mu$ thick with a cross section of about $1.5 \mathrm{~cm}^{2}$ were cut with a string saw so that the (001) basal plane was exposed.

The crystals were polished to a $1 \mu$ finish and then heated in a vacuum oven for four hours at $650^{\circ}$ and $5 \times 10^{-6}$ torr to increase their conductivity. This treatment creates oxygen vacancies which serve as donors. 76

Prior to use they were etched for one hour in conc. $\mathrm{H}_{2} \mathrm{SO}_{4}$. The final surface was glassy smooth with some ripples that were barely visible to the eye.

The concentration of donors in the crystal was established through capacitance measurements to be $5 \times 10^{18} / \mathrm{cc}$. The flat-band potential of these crystals exhibited a frequency dependence over the range of 1 to $10 \mathrm{kHz}$ becoming more negative with increasing frequency. The slope of the resultant Mott-Schottky plots, however, remained constant. A General Radio 1670A impedance bridge was used in conjunction with 
a Hewlett Packard 3310A function generator.

Ohmic contacts to the crystals were made through vacuum deposition of indium. A window of several $\mathrm{mm}^{2}$ was masked during deposition to allow illumination of the semiconductor surface through the electrode.

Adsorption isotherms for the adsorption of rose bengal on these $\mathrm{TiO}_{2}$ single crystals at $21.5^{\circ} \mathrm{C}$ and $36.5^{\circ} \mathrm{C}$ were determined using an elution technique. ${ }^{17}$ The crystals were immersed in dye solution of up to $4.38 \times 10^{-4} \mathrm{M}$ (all of which were $1 \mathrm{M}$ in $\left.\mathrm{KCl}\right)$. The equilibrium time varied with temperature and dye concentration but was always less than 20 minutes. After a 20 minute immersion the crystals were removed from the dye solution and washed with decane in which rose bengal is insoluble.

Absorption spectra of the dye adsorbed onto the surface of the elactrode are required for determination of both quantum efficiency charge injection and adsorption isotherms. In order to increase the measurement precision a stack of three crystals, with six surfaces, was aligned in the beam of a Cary 118 spectrophotometer for absorbance measurements of dye adsorbed on these surfaces. Another stack of three crystals was placed in the reference beam of the spectrophotometer to compensate for the absorbance of the crystals.

The dye adsorbed on these crystals was eluted with methanol and saved. The product of the concentration of the dye in the eluent and its volume yielded a figure for the absolute amount of dye adsorbed on the crystals. Normalized to unit surface area, this figure can be correlated with the integrated absorption of the adsorbed dye. From it, points on an adsorption isotherm can also be obtained. The electrochemical cell was constructed so that the light 
passed through the $\mathrm{TiO}_{2}$ electrode to the active surface before being absorbed in the bulk of the solution. A Pt counter electrode and a saturated calomel electrode used as a reference were separated from the main body of the dye solution by glass frits. Provisions were made for addition of reagents and stirring of the electrolyte with gas. The electrolyte solutions were not deoxygenated in view of the oxygen evolution at the $\mathrm{TiO}_{2}$ surface at anodic bias potentials. A thermal jacket surrounded the cell for temperature control. The cell was shielded by a Faraday cage of copper mesh.

Photocurrent measurements were made with the experimental set up depicted in Fig. 25. A $450 \mathrm{~W}$ Xe high pressure lamp served as a light source; a Bausch and Lomb high intensity monochromator or interference filters were used for wavelength selection. The light was modulated by mechanical chopper at frequencies ranging from 3 to $11 \mathrm{~Hz}$. Between photocurrent measurements the $\mathrm{TiO}_{2}$ electrode was washed in successive baths of acetone and distilled water.

Light intensity was measured with the use of siticon solar cell and a Hewlett-Packard 8330A Radiant Flux Meter with an $8334 \mathrm{~A}$ radiant $\mathrm{Flux}$ Detector. In the experiment requiring signal averaging, a Nicolet NIC-80 computer was used.

\section{Results}

1. Adsorption Measurments

Adsorption isotherms for rose bengal on the $\mathrm{TiO}_{2}$ crystals were obtained at two temperatures $21.5^{\circ} \mathrm{C}$ and $36.5^{\circ} \mathrm{C}$ as shown in figure 39 Langmuir type behavior was observed with plateaus attained at solution

concentrations of about $1 \times 10^{-4} \mathrm{M}$. The information derived from analysis of this isotherm according to the Langmuir expression of eqn. (4) 


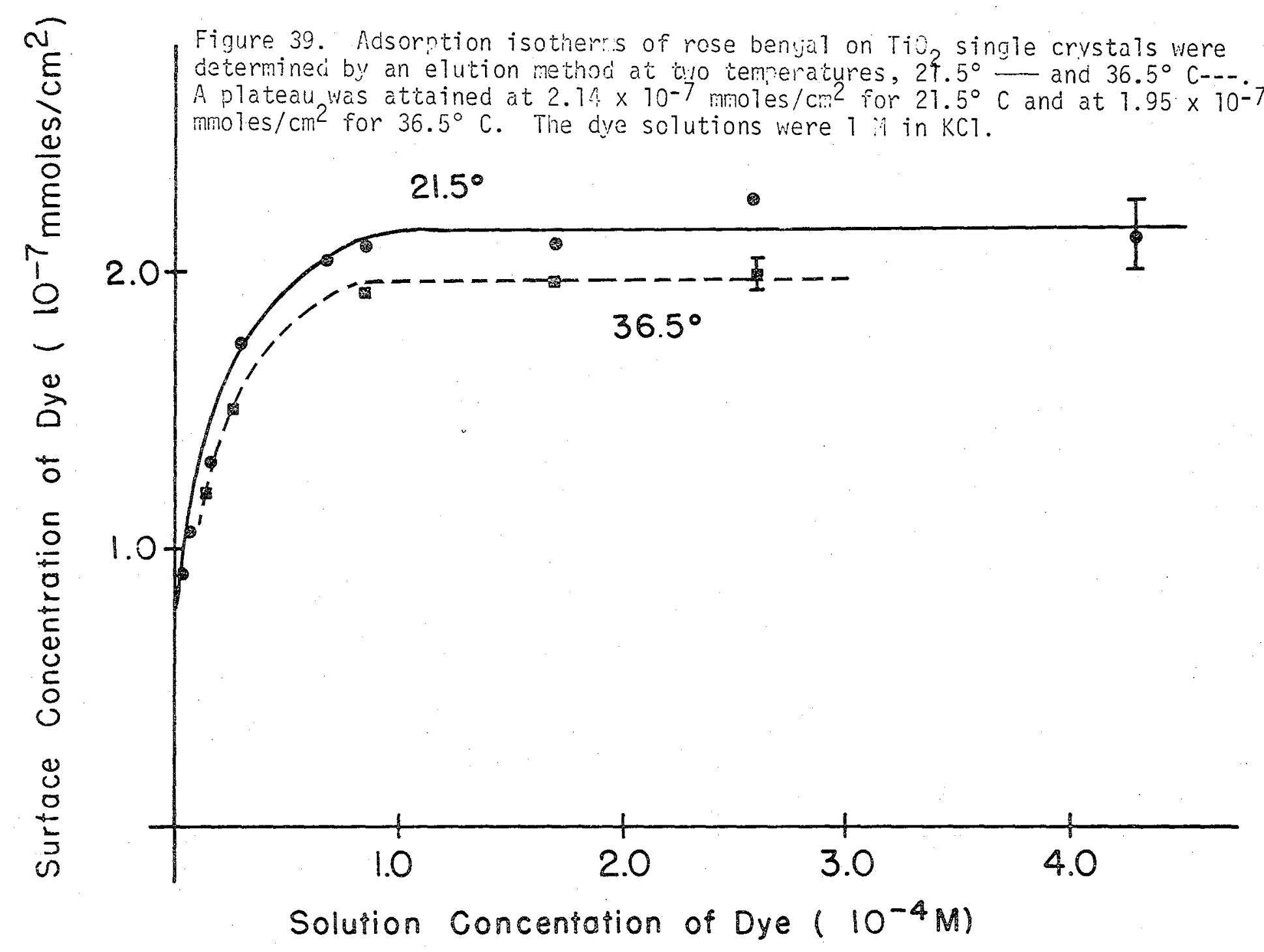


is assembled in Table 7. Assuming a surface with a roughness factor of unity, the area for each molecule can be calculated to be $75 \AA^{2}$ at $21.5^{\circ} \mathrm{C}$ and $81 \AA^{\circ}$ at $35^{\circ} \mathrm{C}$.

Absorbance spectra of the dye adsorbed on the crystal indicate adsorption in predominantly the monomer form. The spectra of figure 40 revealed a gradual increase in surface dimer concentration which is reflected in a greater relative adsorption in the high energy shoulder. Solution spectra of the dye are shown in figure 41 for comparison.

\section{Dye photooxidation}

These $\mathrm{TiO}_{2}$ crystals used in the adsorption measurements were fashioned into electrodes for use in the electrochemical set-up. Immersed in an electrolyte containing the dye, the crystals were illuminated with light in the spectral region of the dye; at anodic potentials an oxidative photocurrent was observed. With this bias sufficient to draw off al1 injected charge, the photocurrent at the absorbance maximum was recorded as a function of time (figure 42). A fast decay was observed which was followed by a slower long term decline in the photocurrent. The decay can be attributed to an accumulation of the oxidized dye on the surface of the electrode with the oxidized form apparently stable enough to permit regeneration of the dye. A coulometric analysis of the current passed through the electrode in the first $500 \mathrm{sec}$ of illumination revealed that only $8 \%$ of the dye on the surface of the electrode has been oxidized. With the electrolyte at $\mathrm{pH} 6.6$ and $21.5^{\circ} \mathrm{C}$, the quantum efficiency the initial charge injection was determined to be $4.0 \times 10^{-3}$. Here the quantum efficiency is defined as the number of electrons transferred 


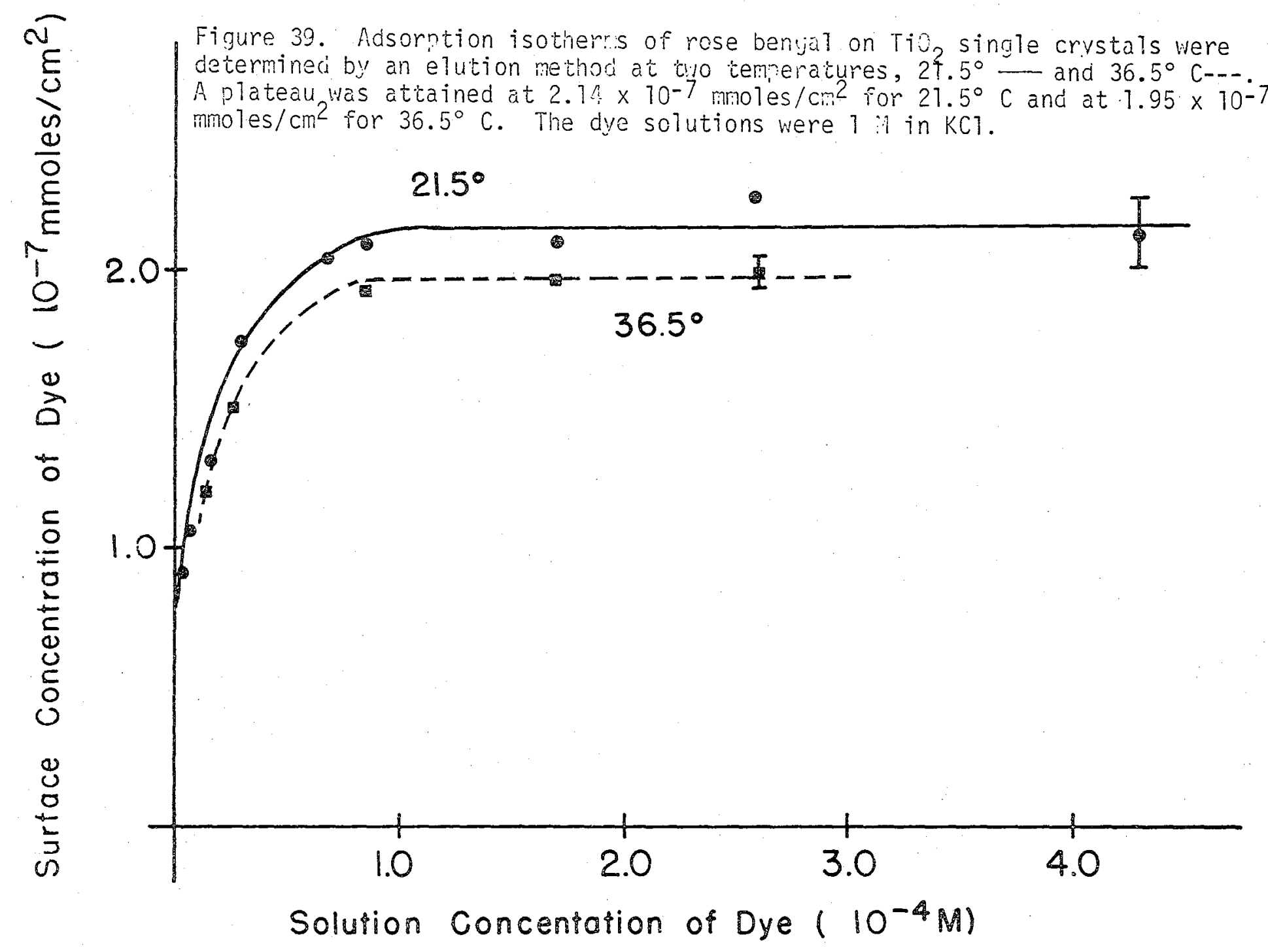


is assembled in Table 7. Assuming a surface with a roughness factor of unity, the area for each molecule can be calculated to be $75 \AA^{2}$ at $21.5^{\circ} \mathrm{C}$ and $81 \mathrm{~A}^{\mathrm{2}}$ at $35^{\circ} \mathrm{C}$.

Absorbance spectra of the dye adsorbed on the crystal indicate adsorption in predominantly the monomer form. The spectra of figure 40 revealed a gradual increase in surface dimer concentration which is reflected in a greater relative adsorption in the high energy shoulder. Solution spectra of the dye are shown in figure 41 for comparison.

2. Dye photooxidation

These $\mathrm{TiO}_{2}$ crystals used in the adsorption measurements were fashioned into electrodes for use in the electrochemical set-up. Immersed in an electrolyte containing the dye, the crystals were illuminated with light in the spectral region of the dye; at anodic potentials an oxidative photocurrent was observed. With this bias sufficient to draw off al1 injected charge, the photocurrent at the absorbance maximum was recorded as a function of time (figure 42). A fast decay was observed which was followed by a slower long term decline in the photocurrent. The decay can be attributed to an accumulation of the oxidized dye on the surface of the electrode with the oxidized form apparently stable enough to permit regeneration of the dye. A coulometric analysis of the current passed through the electrode in the first $500 \mathrm{sec}$ of illumination revealed that only $8 \%$ of the dye on the surface of the electrode has been oxidized. With the electrolyte at $\mathrm{pH} 6.6$ and $21.5^{\circ} \mathrm{C}$, the quantum efficiency the initial charge injection was determined to be $4.0 \times 10^{-3}$. Here the quantum efficiency is defined as the number of electrons transferred 


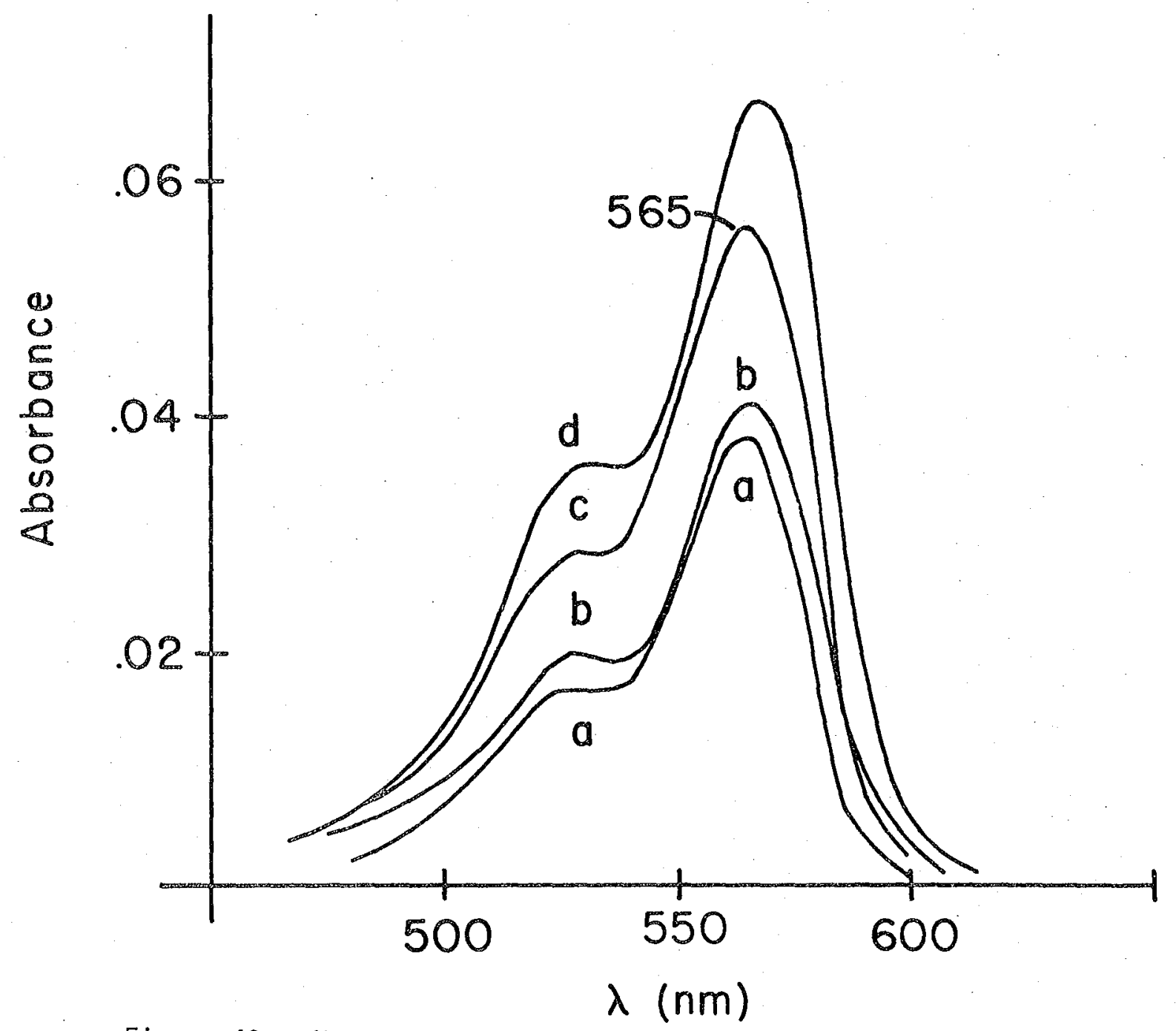

Figure 40. Absorbance spectra of rose bengal adsorbed onto $\mathrm{TiO}_{2}$ single crystals are shown for different surface concentrations. (a) $0.93 \times$ $10^{-7}$ (b) $1.19 \times 10^{-7}$ (c) $1.73 \times 10^{-7}$ (d) $2.13 \times$ $10^{-7}$ mmoles $/ \mathrm{cm}$. These spectra are the sum of the absorbances of six surfaces. 


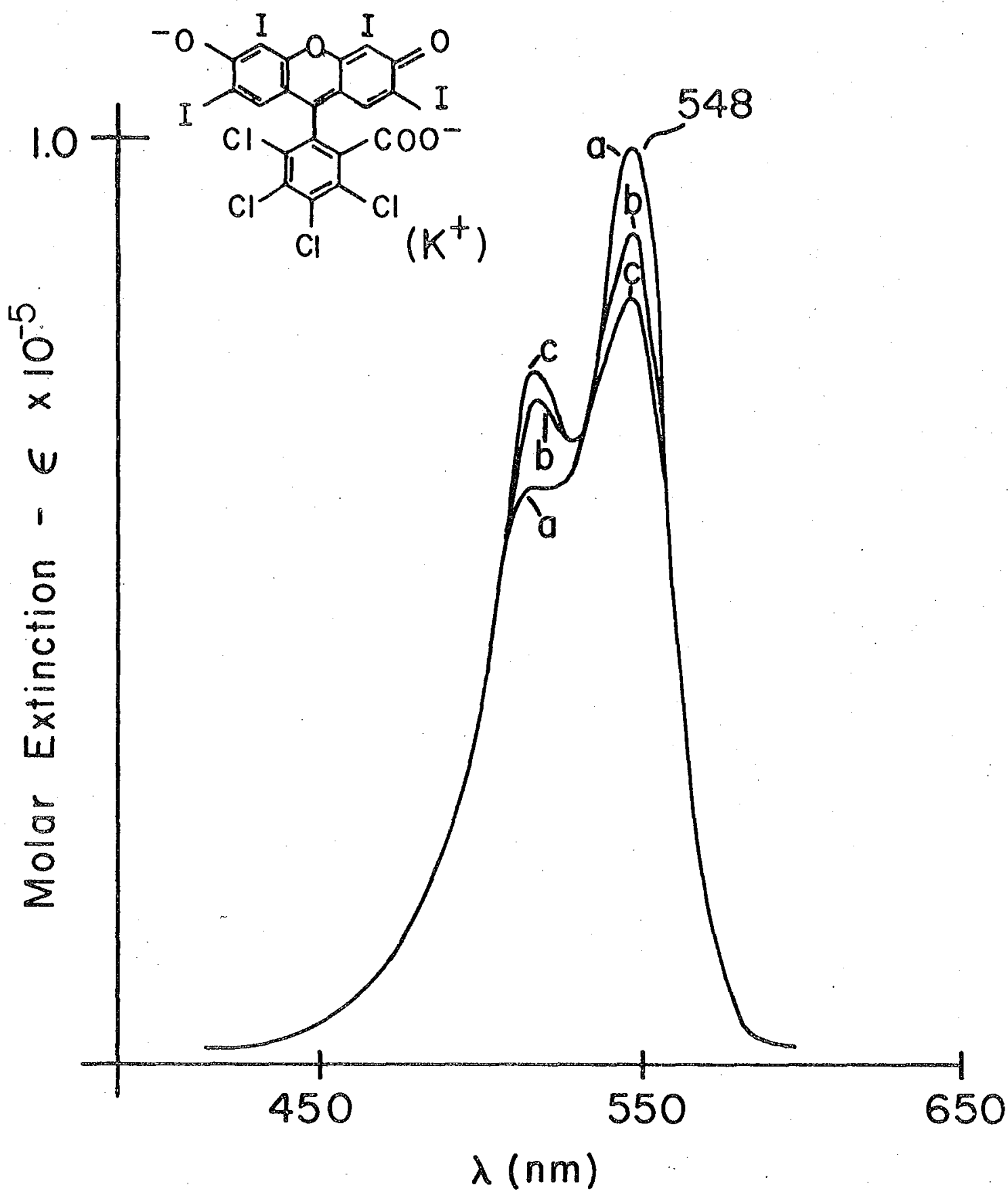

Figure 41. The solution absorbance of rose bengal in 1 i $\mathrm{KCl}$ reveals increasing dimer formation over the range of dye concentrations covered by the adsorption isotherm of Figure 39. Spectrum (a) represents the monomer form with a higher energy shoulder $30 \mathrm{~nm}$ down from the maximum at $540 \mathrm{~nm}$. Spectrun (c) shows the emergence of the dimer maximum at 520 nris. These siectra correspond to dye concentrations of (a) $5.4 \times 10^{-5} \mathrm{i}$ (b) $2.1 \times 10^{-4} \mathrm{il}$, (c) $4.4 \times 10^{-4}$ il. 
Figure 42. (a) The time decay of rose bengal sensitized photocurrents at $\mathrm{TiO}_{2}$ reveals an initial fast decay with a subsequent slow decrease in the rate of photooxidation. 560 nm 7 ight was used with a $3.6 \mathrm{hz}$ modulation

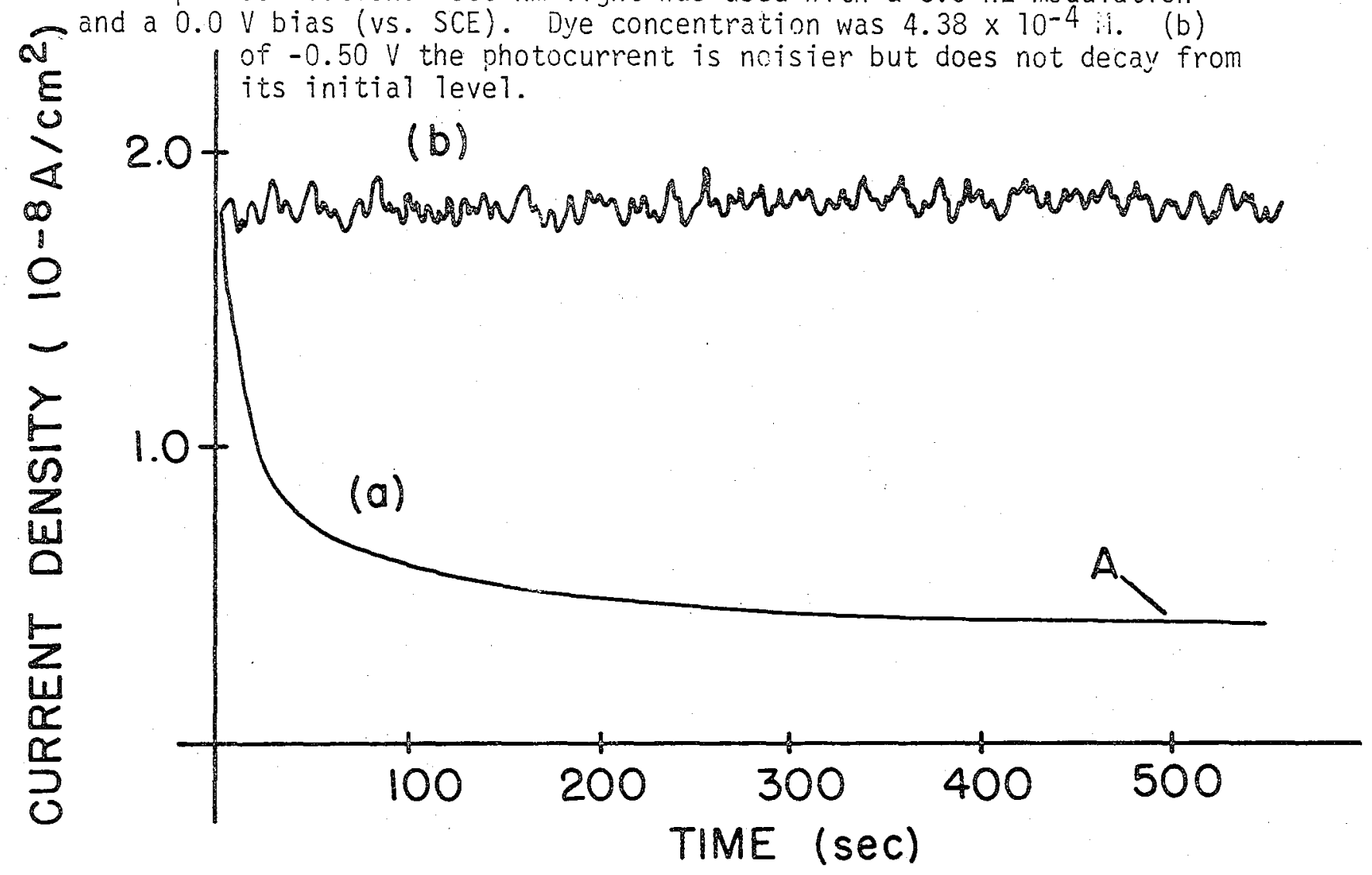

XBL 767-6061 
per photon absorbed by the dye.

After a quasi-steady state was reached in the time decay of the current corresponding to point $A$ of figure 42 , the light was swept through the spectral region of the dye, giving the spectrum of figure 43. This curve compares very well with the absorbance spectra of figure 40 .

In Chapter III it was demonstrated that $\mathrm{Cl}^{-}$functions as a reducing agent for rhodamine $\mathrm{B}$ adsorbed on $\mathrm{ZnO}$. It is necessary to show for the system rose bengal/ $1 \mathrm{M} \mathrm{KCl/} \mathrm{TiO}_{2}$ that chloride does not photoreduce the excited dye since the electrochemical properties of the reduced dye are not of interest in this work.

This was accomplished through the measurement of the quantum efficiency of electron injection as a function of chloride concentration when the electrolyte is $3.2 \times 10^{-5} \mathrm{M}$ in the rose bengal (figure 44). To evaluate the quantum efficiency it was necessary to measure the optical density of the adsorbed dye at each concentration of $\mathrm{KCT}$ and correlate it with the observed photocurrent. The absorbance measurements were taken as described in the experimental section. Photooxidation of rose bengal at $\mathrm{TiO}_{2}$ appears to be insensitive to chloride and the observed photocurrent may be seen to reflect the oxidation of the excited dye at the electrode surface.

With the use of a $560 \mathrm{~nm}$ interference filter, the photocurrent was measured as a function of the solution concentration of the dye (figure 45). Since the absorbance of the adsorbed dye for specific surface concentrations is known, a determination of the quantum efficiency as a function of surface coverage is possible. In figure 46 it can be seen that the efficiency of charge transfer is 


\section{$040047 \% 9$}

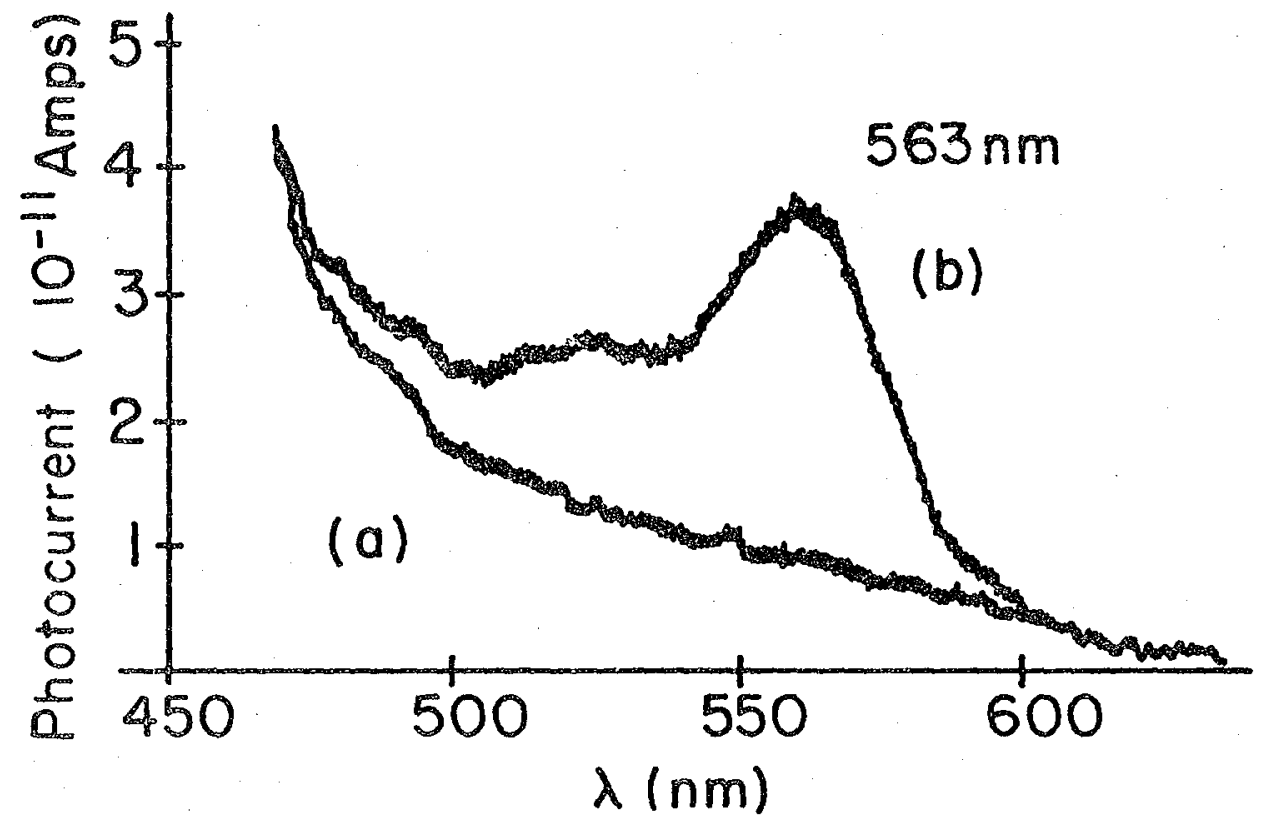

XBL 767-6062

Figure 43. (a) Photocurrent produced by defects in the crystal itself decreased bitin increasing wavelength. This serves as a baseline for the sensitized oxidative photocurrent (b) that occurs when the dye is adsorbed onto the electrode surface. This action spectrum was taken when the photocurrent had reached a decay level equivalent to point $A$ in Fig. 42. The bias was $0.18 \mathrm{~V}$ (SCE). Dye concentration was $4.38 \times 10^{-4} \mathrm{M}$. 

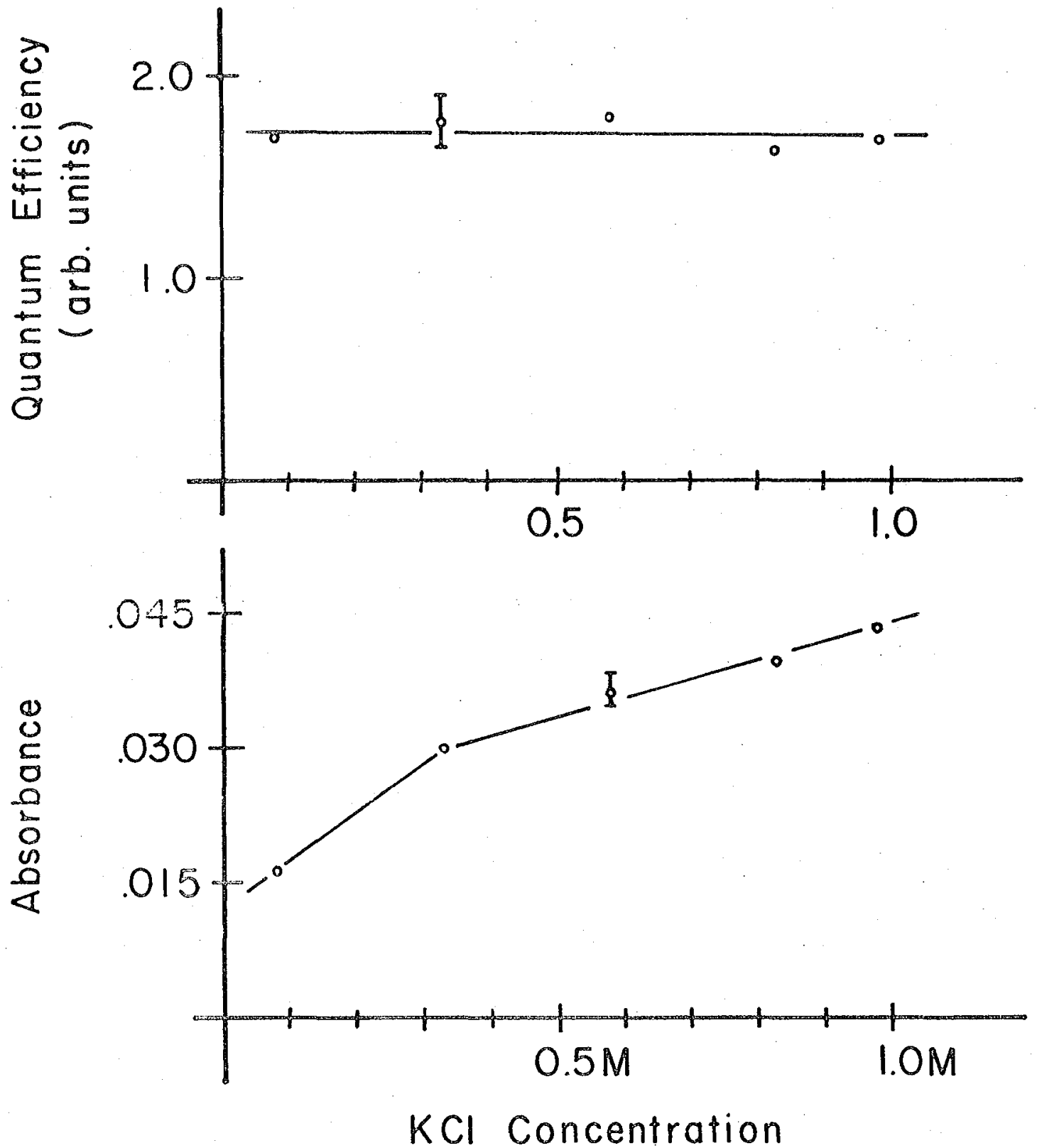

Figure 44. Although chloride functions as a

XBL $7611-9664$ reducing agent for excited dyes on the surface of $\mathrm{ZnO}$, it can be sien here that chloride does not affect the quantum efficiency of charge transier from rose bengal to $\mathrm{TiO}_{2}$ over a concentration range of .08 il to 1.0 il. 


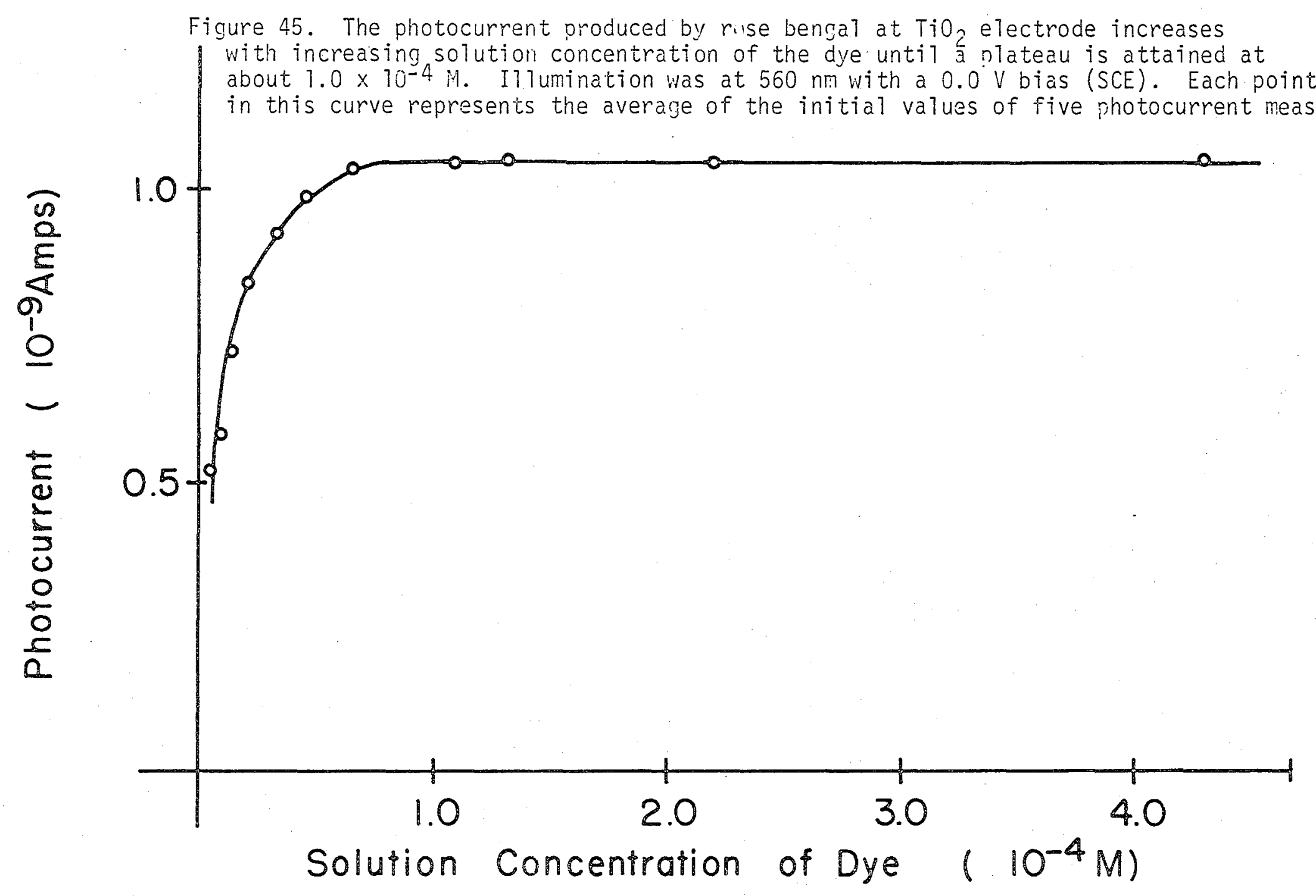

XBL 767-6051 


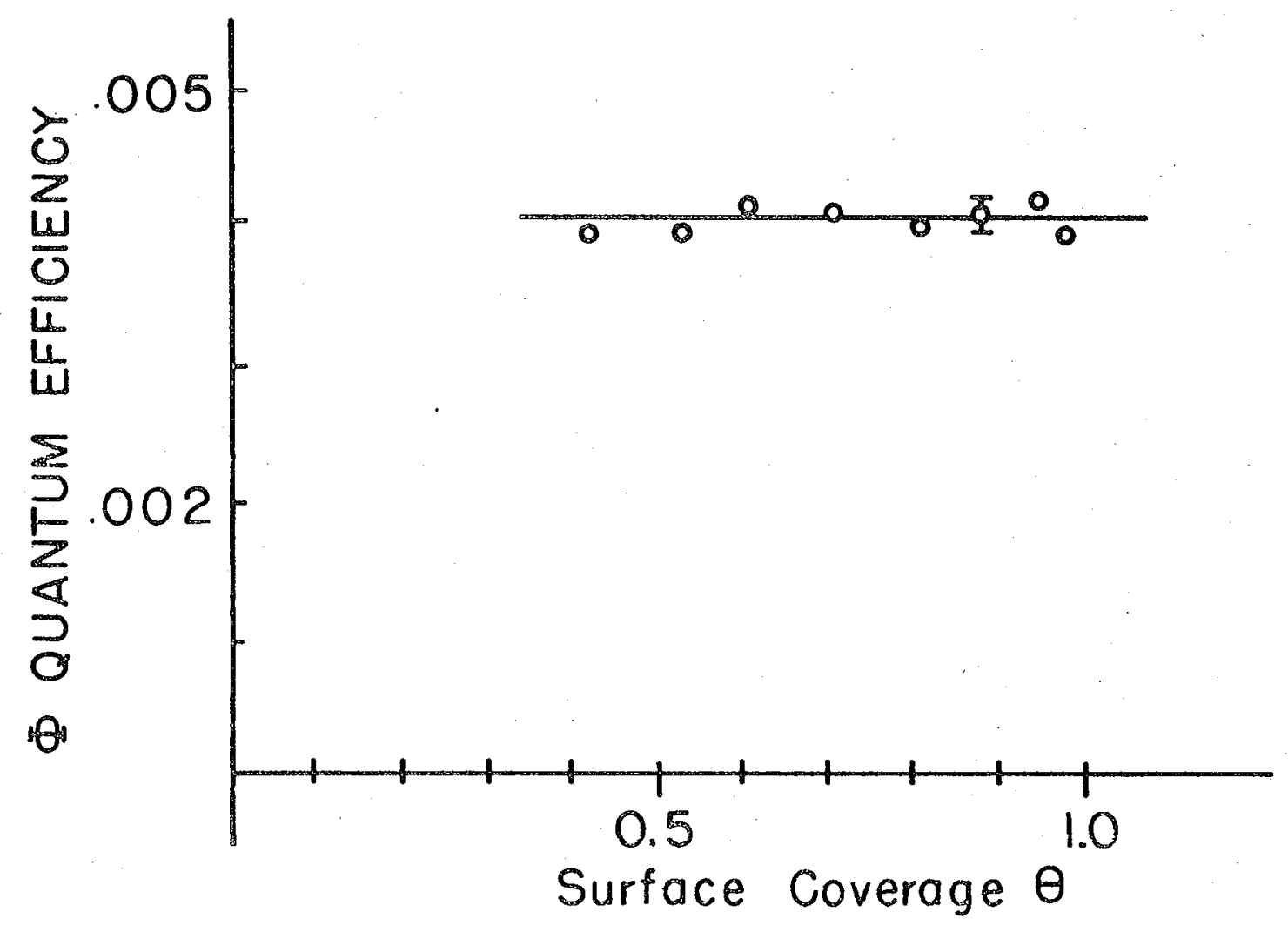

Figure 46. The quantum of electron transfer was found to be independent of the surface concentration of the dye down to about $40 \%$ coverage of the electrode. This graph was constructed from data like those depicted in Figures 40 and $45 . \theta=1.0$ corresponds to $a$ surface coverage of $2.2 \times 10^{-7} \mathrm{mmoles} / \mathrm{cm}^{2}$. 
independent of surface concentration of the dye over the range investigated.

The dye molecule is adsorbed on the surface of the $\mathrm{TiO}_{2}$ in the Helmholtz region of the double layer. Except when the semiconductor becomes degenerate, there is a negligible change of the potential drop across the Helmholtz layer with a change in the polarization of the electrode. However, through capacitance measurements this drop has been found to be dependent upon the pH of the electrolyte solution; for $\mathrm{TiO}_{2}$, it has been reported to change by about $60 \mathrm{mV} / \mathrm{pH}$ unit. 77

In order to find the extent to which a change in the Helmholtz potential drop affects the electron transfer reaction, the photocurrent was measured as a function of $\mathrm{pH}$. This experiment has been done with rhodamine on $\mathrm{ZnO}^{15}$ and is conceptually similar to currentvoltage curves for reactions at metal electrodes. Measurements were taken from $\mathrm{pH} 5$ to 11 ; acidic $\mathrm{pH}^{\prime} \mathrm{s}$ were avoided Decause of protonation of the dye. A logarithmic plot of the photocurrent is seen in figure 47 as a linear function of $\mathrm{pH}$ with an average slope of .07 \pm .01 .

The absorbance of the adsorbed dye layer was also measured as a function of $\mathrm{pH}$ and was found to describe a similar logarithmic relation with siope $.07 \pm .01$. The quantum efficiency of electron transfer must then be independent of the change in the potential drop across the Helmholtz region over the range covered by these $\mathrm{pH}$ experiments. The spectral characteristics of the dye were also examined as a function of $\mathrm{pH}$. Absorption and fluorescence spectra exhibited no significant change over the $\mathrm{pH}$ range of interest 

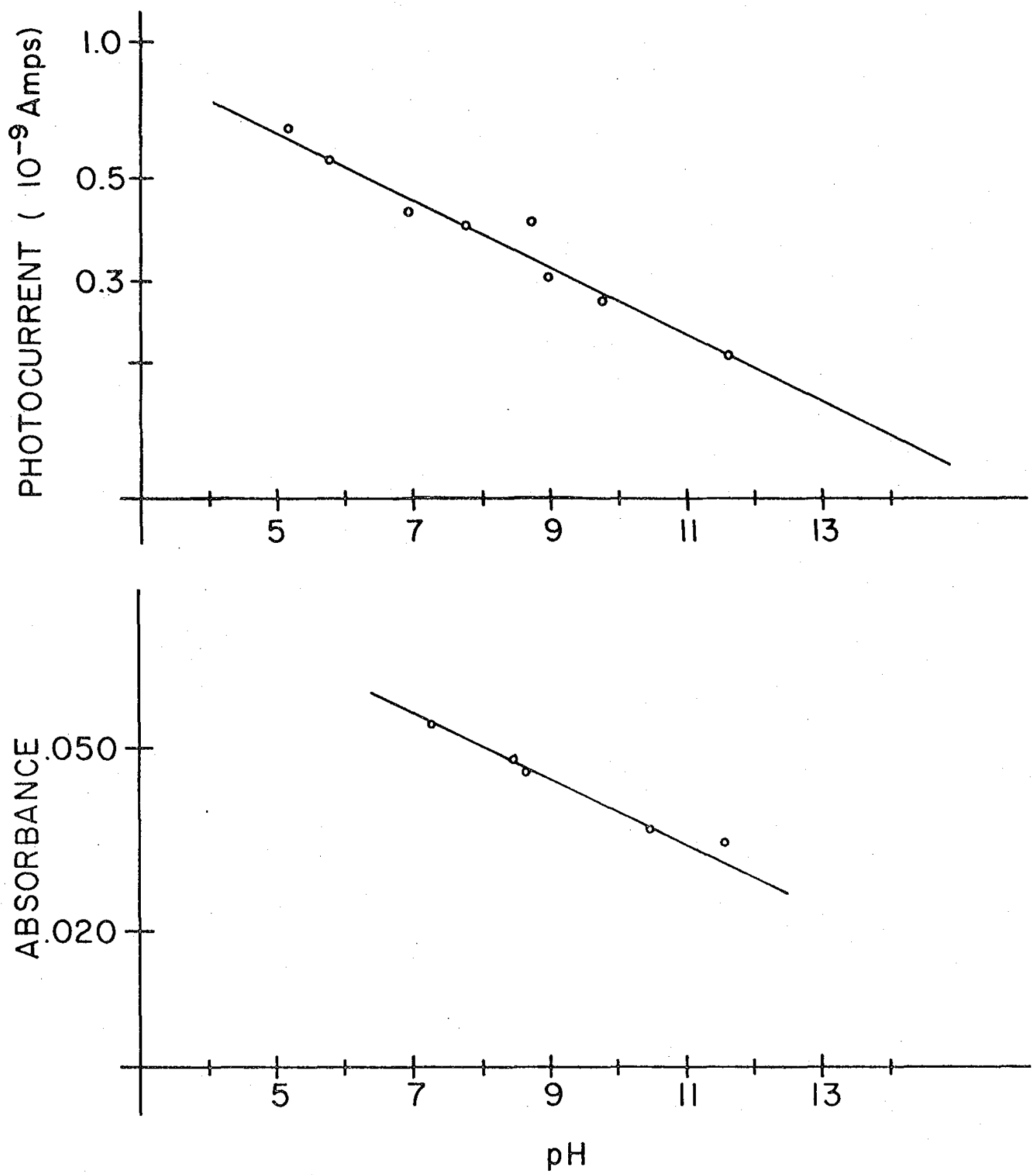

Figure 47. (a) a Logarithmic analys is of the photocurrent as a function of $\mathrm{pH}$ yielded a line with an average slope of $.07 \pm .01$. (b) The $\mathrm{pH}$ of the solution affects the photocurrent because of the $\mathrm{pH}$ dependent adsorption characteristics of the dye. From a basic electrolyte less dye is adsorbed on the surface than from neutral or acidic solutions. A slope of .07 \pm .01 was also found for this measurement. 


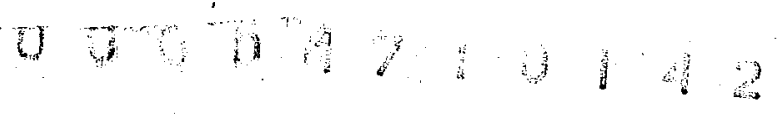

indicating no $\mathrm{pH}$ dependent properties of the ground or excited state of the dye.

3. Temperature Dependence

Activation energy determinations in spectral sensitization have been confined to systems where the dye was adsorbed at a gassolid interface or was applied to the solid as a thin film. In electrochemical systems some difficulties are encountered in the experimental determination of an activation energy. The potential of the reference electrode is a temperature dependent quantity, a condition which can hinder comparison of current densities at different temperatures. At sensitized electrodes, the use of water soluble dyes brings in another temperature dependent variable in the form of an adsorption isotherm.

As described earlier, adsorption information for rose bengal on $\mathrm{TiO}_{2}$ at two temperatures was determined. It is important, too, to reiterate that the quantum efficiency of charge injection is not sensitive to the change in surface concentration that occurs between these two temperatures. Also, a change in the reference potential is unimportant because the semiconductor electrode can be polarized in a saturation region where a change in bias does not affect the photocurrent. An additional complication in a temperature study would be a temperature dependent potential drop across the Helmholz layer at the electrode surface. Through capacitance measurements, this change in the Helmholtz potential drop at $\mathrm{TiO}_{2}$ was measured to be $\pm 0.10 \mathrm{~V}$ over the $15^{\circ}$ range between $21.5^{\circ}$ and $36.5^{\circ} \mathrm{C}$. Through the $\mathrm{pH}$ study above, however, the effect of such a change in the Helmholtz region on the quantum efficiency is known to be negligible. With 
these variables determined, it should be possible to obtain an activation enthalpy for the photooxidation of rose bengal at $\mathrm{TiO}_{2}$ electrode.

In order to derive this quantity from photocurrent measurements, it is necessary to compute the temperature dependent quantum efficiency, $\Phi(T)$ :

$$
\Phi(T)=I(T) / n \cdot A(T)
$$

where $T$ is the temperature; $I(T)$ is the current density at $T$ in electrons $\mathrm{cm}^{-2} \mathrm{sec}^{-1} ; A(T)$ is the absorption of the dye adsorbed on the electrode; $n$ is the photon flux in photons $\mathrm{cm}^{-2} \mathrm{sec}^{-1}$.

Values for $A(T)$ were taken from the absorbance spectra used to construct the isotherms of figure 39. Interpolation between the absorbances of $21.5^{\circ} \mathrm{C}$ and $36.5^{\circ} \mathrm{C}$ yields figures for $\mathrm{A}(\mathrm{T})$ at intermediate temperatures. I $(T)$ was taken as the initial magnitude of the photocurrent before the time decay set in. A plot of the logarithm of $\Phi(T)$ vs inverse temperature should yield an activation enthalpy for the electron transfer.

The temperature dependence of the photocurrent between $21.5^{\circ} \mathrm{C}$ and $37.5^{\circ} \mathrm{C}$ was measured. $\Phi(T)$ was computed for each experimental point and used to construct the Arrhenius plot of figure 48. A slope of $6.7 \pm .5 \mathrm{kcals} / \mathrm{mole}$ was obtained with a least mean squares fit. The pre-exponential factor was $4 \times 10^{3}$ within a factor of two.

These measurements began at room temperature; the solution was then heated to $37^{\circ} \mathrm{C}$ and data were taken as the solution cooled. Between measurements the $\mathrm{TiO}_{2}$ electrode was washed in successive baths of acetone and distilled water. A twenty minute equilibration period in the dye solution was allowed prior to illumination; the 
Figure 48. An Arrhenius plot of the quantum efficiency for photooxidation of rose bengal yields an activation enthalpy of $6.7 \pm .5 \mathrm{kcal} / \mathrm{mole}$. Each data points represents a single experimental measurement; illumination was at $560 \mathrm{~nm}$ with a $0.20 \mathrm{~V}$ bias on the electrode. The rose bengal concentration was $7.6 \times 10^{-4} \mathrm{M}$. The preexponential factor was $4 \times 10^{3}$.

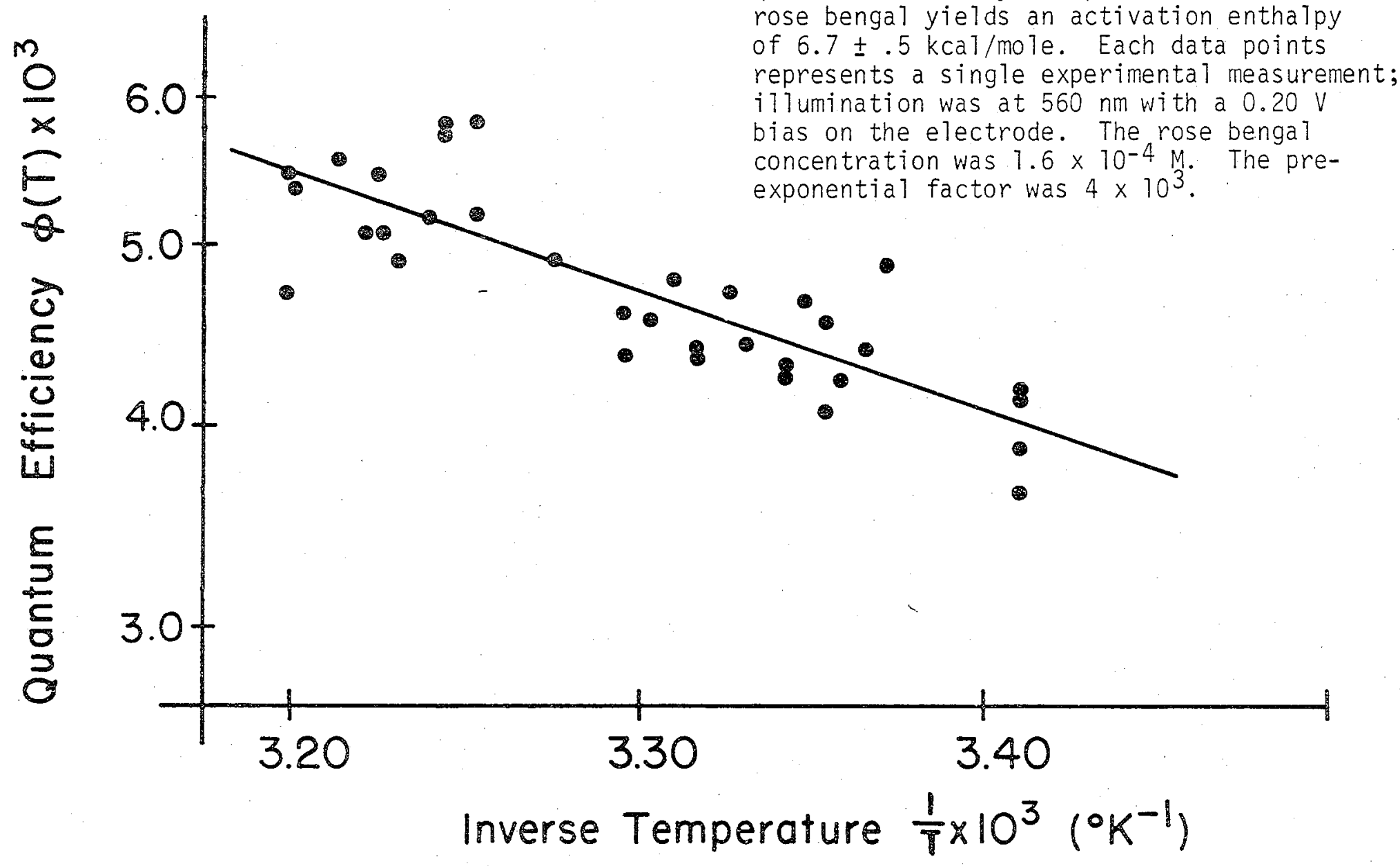


acquisition of the data in figure 11 required several experimental runs.

4. Sensitized photoreduction

The commonly observed bias dependence of the photocurrent from a dye-semiconductor pair can be seen in figure 31 for rhodamine $B$ adsorbed on $\mathrm{ZnO}$. The current rises from zero at cathodic potentials to saturation at a more anodic bias. Since $K C l$ was used as the electrolyte in this system, it is the reduced rhodamine $B$ that injects the electron to produce the current depicted in this figure.

In the system of rose benga $]$ and $\mathrm{TiO}_{2}$, however, the bias dependence of the photocurrent is characterized by the unusual features depicted in figure 49. Instead of the photocurrent disappearing at lower potentials, a reduction current set in which peaked at about $-0.85 \mathrm{~V}$.

The magnitude of this reduction reaction at $-0.85 \mathrm{~V}$ was found to be linearly dependent upon the absorbance of the dye in solution. An action spectrum at this peak corresponds to the absorbance of the dye in solution depicted in figure 41. Stirring of the electrolyte increased the reduction photocurrent while deoxygenating the solution with $\mathrm{N}_{2}$ greatly diminshed it. Introduction of hydroquinone also resulted in a decrease of the reduction current. Stirring of the solution with air increased the current, but a subsequent changeover of stirring gases to pure $\mathrm{O}_{2}$ did not result in any further increase. Thus, the concentration of excited dye in solution appears to be the rate limiting species. This reduction reaction could never be eliminated, presumably because of the dark current evolution of $0_{2}$ at the $\mathrm{TiO}_{2}$ surface. 


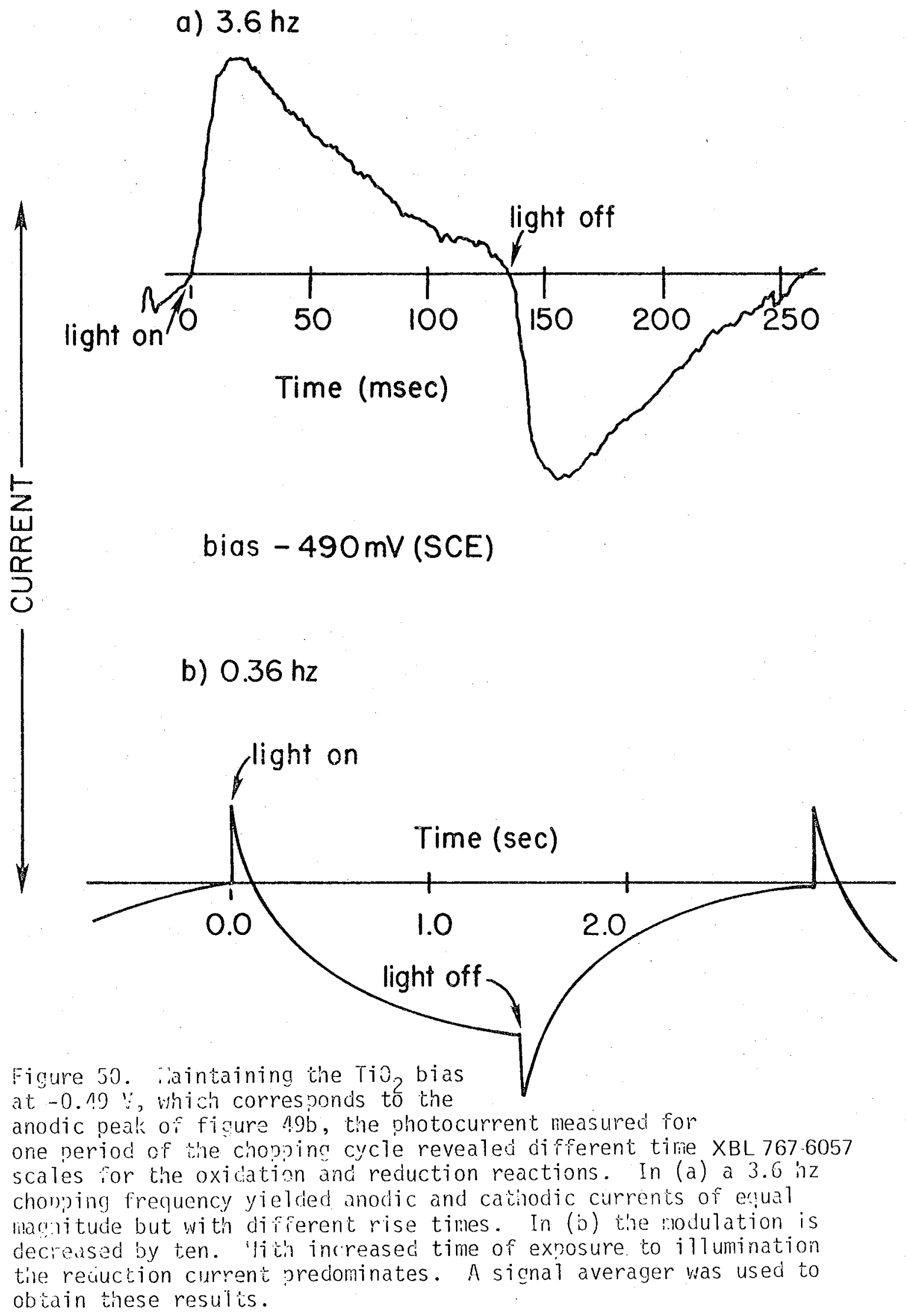


bengall.

At a bias potential more negative than $-0.24 \mathrm{~V}$ the net current flowing across the $\mathrm{TiO}_{2}$ surface reflects contributions from several reactions, the dye sensitized oxidation and reduction, reduction of oxygen, and hydrogen and oxygen evolution from the conduction and valence bands of the semiconductor. These partial currents are depicted in figure 51 .

The dark current at the electrode (figure 51a) reflects the evoTution and reduction of oxygen at the surface and at more negative potentials, evolution of hydrogen. The potential dependence of oxygen evolution at the electrode (figure $5 \mathrm{lb}$ ) can be seen in the currentvoltage characteristics of the $\mathrm{TiO}_{2}$ intrinsic photocurrent. The photomodulated sensitized oxidation and reduction currents are superimposed upon these two background reactions. The influence of the dark reactions on the sensitized photocurrent is clear.

\section{Discussion \\ 1. Adsorption Is otherm}

From the absorbance spectra of the dye adsorbed on the $\mathrm{TiO}_{2}$ crystals it is evident that the dye is adsorbed from solution in the monomer form. The spectra in figure 40 reflect a progressively larger fraction of surface coverage with a relatively small increase of $20 \%$ in the high energy shoulder where dimer absorbance occurs. This increase in surface dimerization can probably be attributed to the increasing concentration of the dimer in solution.

An estimate of the heat of adsorption of the dye may be made with data obtained from analysis of the adsorption isotherms. Using a $10 \mathrm{~A}$ as an estimate for $D$ in eqn. (4a), a $\Delta H$ of approximately $7.6 \mathrm{kcal} / \mathrm{mole}$ 


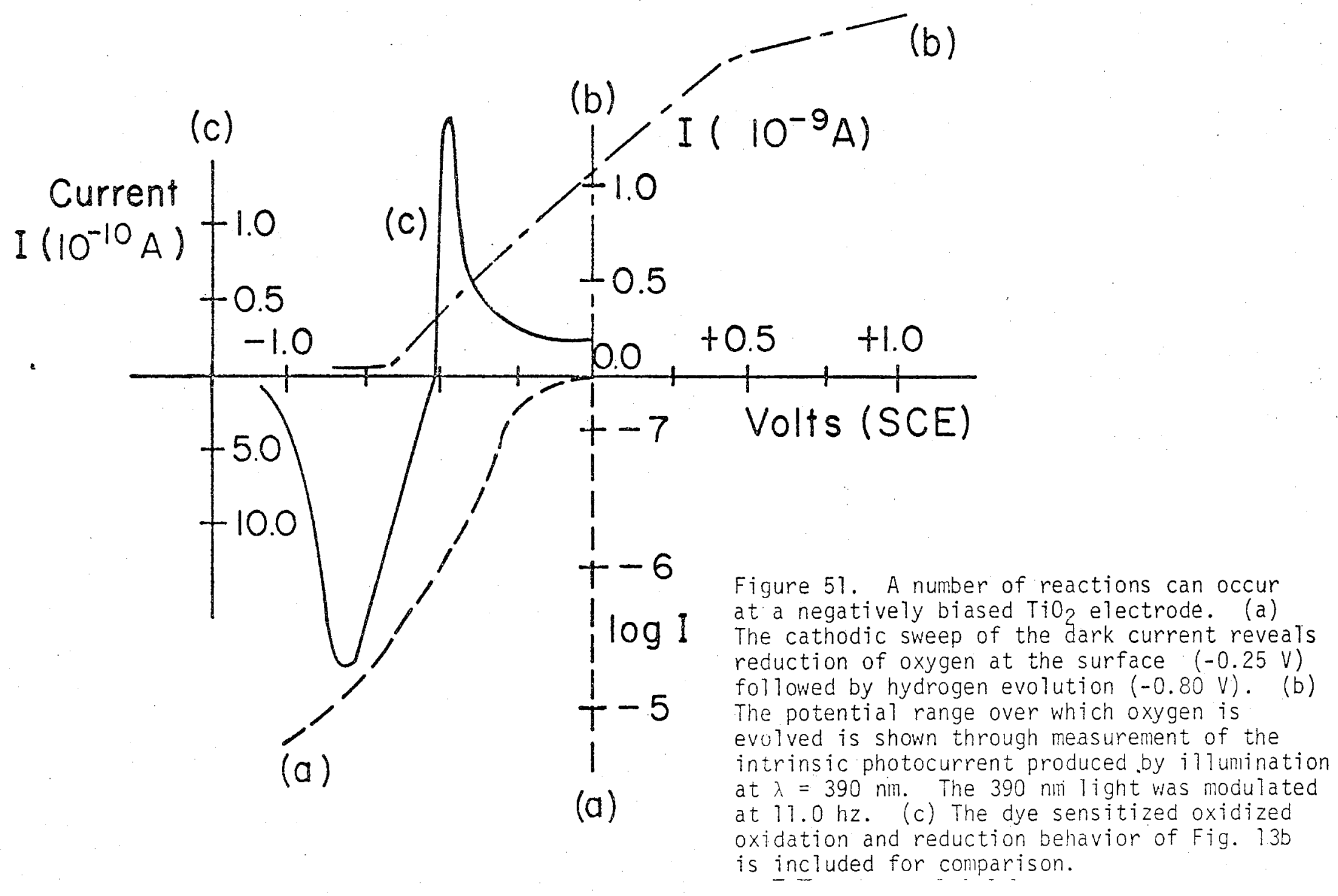


is obtained for both temperatures (see Table 6 ). As discussed in

Chapter II, a $\Delta H$ of this size is considered large enough to insure monolayer coverage of the surface.

Implicit in the use of a Langmuir analysis of an adsorption isotherm is the assumption that $\Delta H$ is independent of surface coverage. This is probably true for the surface concentrations covered by these experiments, However, the data only extended down to a coverage of $\theta=0.4$ so that use of this Langmuir analys is places no a priori assumptions on variations in $\Delta H$ below this limit. Indeed, it has long been established ${ }^{78}$ that the heat of adsorption is greatest for the first additions of dye to the surface. Physically, this higher $\Delta H$ can be seen as the manifestation of a small number of more active adsorption sites which are preferentially occupied at low surface coverage. $\Delta H$ subsequently decreases as $\Theta$ becomes larger with the differential heat of adsorption eventually tending to zero. It is not unreasonable to expect a similar $\theta$ dependence of $\Delta H$ for the adsorption of rose bengal on $\mathrm{TiO}_{2}$ at small $\Theta$.

A complete coverage of the surface appears probable upon examination of the experimentally determined surface area/unit dye molecule. At $21.5^{\circ} \mathrm{C}$ with an assumed surface roughness factor of unity, each dye molecule occupies $75 \AA^{2}$ of the surface. Using models, a maximum of $100 \AA^{2}$ is expected for this dye. This would imply tight packing of the dye and, indeed, some increase in dimerization is present in the absorbance spectra. However, some degree of roughness greater than unity can be expected and the existence of a plateau in the isotherm suggests a not unreasonable minimum value of 1.3 for this etched surface.

Excitation of the rose bengal at the $\mathrm{TiO}_{2}$ surface results in 
Table 6. Calculated values for the rose bengal adsorption isotherms of Figure 39.

\begin{tabular}{|c|c|c|c|}
\hline $1 / C_{m}$ & $4.57 \times 10^{6} \pm .07$ & $\mathrm{~cm}^{2} / \mathrm{mmole}$ & $4.85 \times 10^{6} \pm .04$ \\
\hline $1 / c_{m} B$ & $27 . \pm 13$ & $\mathrm{~cm}^{2} / \mathrm{ml}$ & $4.1 \pm 7$ \\
\hline$C_{m}$ & $2.19 \times 10^{-7} \pm .04$ & mmole $/ \mathrm{cm}^{2}$ & $2.05 \times 10^{-7} \pm .02$ \\
\hline $1 / B$ & $6 \times 10^{-6} \pm 3$ & mmole $\mathrm{cm}^{3}$ & $8 \times 10^{-6}+.02$ \\
\hline Area & 75 & $\AA^{\circ} 2 /$ molecule & 81 \\
\hline$\Delta H$ & $7.3-7.9$ & $\mathrm{kcal} / \mathrm{mole}$ & $7.5-7.7$ \\
\hline
\end{tabular}


generation of an oxidative photocurrent with action spectra (figure

43) closely matching the absorbance of the adsorbed dye (figure 40). The distinct difference between these spectra and the solution spectra of the dye excludes the probability of participation of solution species in the photooxidation reaction.

This comparison also shows that the surface spectra maxima are shifted about $15 \mathrm{~nm}$ to the red from the corresponding solution peaks. If adsorption of the dye were due entirely to dispersion forces, an absorbance maximum at the wavelength of the peak photocurrent response

in figure 43 would yield a value for the index of refraction of $n_{d} \sim 1.4$ for the semiconductor surface. This would imply that the optical dielectric constant of the adsorbed layer is larger than that for the solution bulk.

\section{Excitation Transfer}

The time decay of the photocurrent reveals that after a 500 second exposure to $560 \mathrm{~nm}$ light only $8 \%$ of the dye on the surface has. been oxidized. Evidently, very few of the excited, adsorbed molecules are able to inject an electron into the crystal while in an excited state.

It is probable that the adsorption site of the dye plays a critical role in determining its ability to sensitize the substrate. Given a dye at the electrode surface, its adsorption orientation and its distance from the surface should vary according to the molecular details of its adsorption site. These details, for example, will be closely tied to the dye molecule's heat of adsorption. Those molecules with a high heat of adsorption will be more tightly bound to their sites and will be closer to the surface than those with lower adsorption heats. Less favorable sites bind the dye molecule more loosely and 
thereby would have less of an influence upon the adsorption orientation of the molecule. Consequently, dye molecules at fifferent sites on the surface may be expected to exibit different efficiencies of electron transfer.

This argument is a microscopic examination of the primary assumption in the kinetic analysis of the time decay discussed in Chapter III: not all dye molecules adsorbed on the semiconductor surface exhibit the same efficiency of sensitization. This condition is more apparent in this system than at the rhodamine $\mathrm{B} / \mathrm{ZnO}$ interface since such a small percentage of the adsorbed rose bengal molecules participate in the photooxidation reaction.

In figure 42 a rapid decline of the photocurrent is seen which is followed by a slower, long term decay. Thus. those molecules on "reactive" sites which permit high quantum efficiencies of electron transfer can be seen to be quickly oxidized with the subsequent photocurrent dye mainly to dye on "reactive" sites with lower quantum efficiencies. The remainder of the adsorbed dye must then be adsorbed at nonreactive locations.

To determine if there was excitation transfer among the dye molecules in the adsorbed layer, the quantum efficiency of electron transfer was calculated as a function of surface coverage just as was done in Chapter III. Once again the absorbance aspectra of the adsorbed dye is evidence that such excitation transfer could only be that resulting from a very weak interaction.

The data of figure 46 demonstrate that the quantum efficiency of charge injection remains constant over a range of surface coverage from 1.0 to $2.2 \times 10^{-7} \mathrm{mmole} / \mathrm{cm}^{2}$. These results are much more convincing than the data for rhodamine $B$ on $\mathrm{ZnO}$ since the per cent mean 
standard deviation is $5 \%$ as compared with $7 \%$ for rhodamine $B / K C 1$

and $17 \%$ for rhodamine $\mathrm{B} / \mathrm{KiNO}_{3}$. However, the question of the existence of excitation transfer is not unequivocably determined. Such an energy transfer could be negligible or its effect on $\Phi$ could saturate before the coverage of $1.0 \times 10^{-7} \mathrm{mmole} / \mathrm{cm}^{2}$ has been reached. This coverage, $\theta=0.4$, is much larger than the previously established limit of $\Theta=0.01$ which must be reached in order to make an absolute dtermination of the existence of excitation transfer.

Further examination of the data in figure 46 , however, provides a basis for postulating the existence of excitation transfer among the dye molecules on the semiconductor surface. It must be assumed, though, that the efficiency of electron transfer from the dye to the semiconductor is dependent on its site of adsorption and therby on its energy of adsorption.

It has has been discussed previously how a measure of this energy, i.e., the heat of adsorption, can be expected to decrease with increasing coverage of the surface with the last molecules adsorbed having the smallest heat of adsorption. Yet, the data of figure 46 show that the quantum efficiency of charge injection remains constant as an increasing number of the most weakly bound molecules are removed.

This experimental result is compatible with the above assumption only if the probability of excitation of a molecule at a reactive site is directly proportional to the number of quanta absorbed by the dye later on the electrode surface. This implies transfer of excitation from nonreactive molecules to reactive molecules even at $\theta=0.4$ where the intermolecular separation is $13 \AA$.

The greater part of the dye on the surface must then function as an antenna which absorbs the quanta of $1 \mathrm{ight}$ and transfers the 
excitation to reactive sites where oxidation can occur.

\section{Dye-Semi conductor Energetics}

There are two main components in theoretical expressions 76 for the activation energy of an electron transfer reaction. One deals with the energy required to rearrange, or polarize, the environment about the electron so that transfer may occur. The other accounts for the work necessary to bring the reactants together within the required distance and in proper orientation to allow the electronic interaction necessary for transfer.

For the case of dye sensitized semiconductors, a relation describing the first of these contributions to the activation energy ahs been suggested by Gerischer 48 :

$$
J \propto \exp \frac{\left(-\left(E_{C}-E_{D}-E^{*}\right)^{2}\right)}{4 \lambda^{*} k T}
$$

where $E_{C}$ is the energy of the semiconductor conduction band; $E_{D}$ is the energy level of the ground state of the adsorbed dye; $E^{*}$ is the excitation energy; $\lambda^{*}$ is the energy required to rearrange the atoms of the solvent molecules surrounding the excited dye so that they occupy positions compatible with the product species at equilibrium. These terms are pictorally defined in figure 52. The activation energy defined by eqn. (28) is $\Delta E^{2} / 4 \lambda^{*}$ where $\Delta E=\left(E_{C}-E_{D}-E^{*}\right)$ is the energy difference between the conduction band and the maximum of the excited single state of the dve on the surface.

As figure 52 shows, the molecular orbital energies of the dye are distributed about energy maxima owing to thermal fluctuations in the interactions of the molecule with the solvent. This time dependent variation in the positions of the atoms in the species which form the environment about the molecule of interest induces corresponding time dependent changes in the energy of its molecular orbitals. The range 
of this variation in the energy of the molecular orbital can be described by a distribution function ${ }^{48}$ :

$$
W(E)=\frac{1}{4 \pi \lambda^{*} k T} \exp \left(\frac{-\left(E-E_{D}\right)^{2}}{4 \lambda \lambda^{*} k T}\right)
$$

The overlap of this energy distribution for the excited state with the density of states of the semiconductor conduction band will dtermine, according to eqn. (28), the rate of electron transfer from the excited dye to the semiconductor. If $E_{D}{ }^{*}$ rests below $E_{C}$ in figure 52, this overlap will be small and there will be a minimum rearrangement energy, $\Delta E$, required of the environment about the dye for activation of the occupied electronic orbital to $E_{C}$ from $i$ ts mean value $E_{D}{ }^{*}$. In the case where $E_{D}{ }^{*}$ is above $E_{C}$ there will be no minimum polarization energy necessary for electron transfer and no contribution to the activation energy from this source.

The relative values of $E_{C}$ for $\mathrm{TiO}_{2}$ and $E_{D}{ }^{*}$ for the dye in solution can be obtained easily. This is done through comparison of the reduction potettial of the dye, $E_{R}=-1.08 \mathrm{~V}$, and the flat band potential of the semiconductor which indirectly yields the energy of the conduction band, $E_{C}=-0.5 \mathrm{~V}$ (taken from literature sources ${ }^{80}$ because a frequency independent flatband potential could not be obtained from capacitance measurements of these crystals). As soen in figure $52, E_{C}$ rests below $E_{D}{ }^{*}$ for the solution dye species.

For the adsorbed dye, the energy difference between $E_{C}$ and $E_{D}{ }^{*}$ will not be equivalent to that for the solution species, as is depicted in figure 52 in the extreme limit where $E_{D}{ }^{*} E_{C}$. This difference between adsorbed and solution species will depend on the potential drop across the Helmholtz layer at the surface and the position of the dye within this layer. 
Figure 52. An analysis of the energetics of a dye-semiconductor pair is presented here. The ordinate represents an energy scale with the abscissa providing a measure of the density of states of the semiconductor and a distribution function for the dye as expressed by Eq. (29). The lowest level of the conduction band is labeled $E_{C}$ with the means of the ground and excited state distribution of the adsorbed dye labeled $E_{D}$ and $E_{D}{ }^{*}$. The reduction potential for the solution dye, $E_{R}$, yields a value for $E_{D} * S 0 l$. This distribution is placed on a scale with the estimates of the oxidation potentials, $D^{+} / D$, of some of the dyes which sensitized the photoreduction at $\mathrm{TiO}_{2}$ : rose benga 1 ( $R B)$, acridine orange $(A 0)$, thionine $(\mathrm{TH})$.

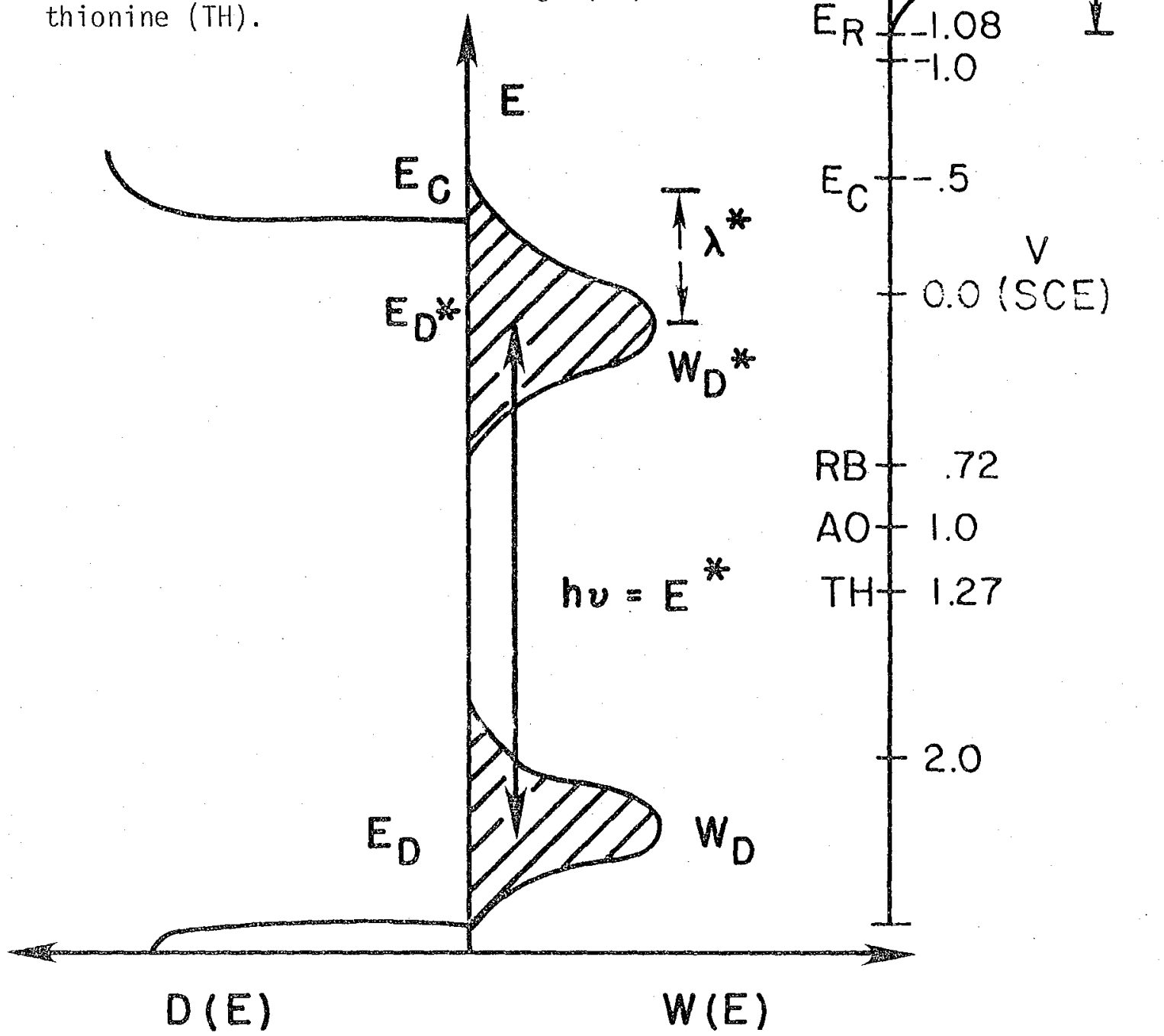


A change in the potential drop across the Helmholtz layer through variation of the $\mathrm{pH}$ of the electrolyte will thereby change $\Delta E$ for the surface dye. Varying $\Delta E$ changes the free energy of the reaction; the magnitude of this $\Delta G$ change will depend on the fractional distance, $\alpha$, relative to the Helmholtz layer thickness, from the electrode surface to the plane of the chromophore. According to eqn. (28), if $E_{D^{*}}>E_{C}$, a change in $\Delta E$ results in an exponential change in the electron transfer rate. 81

In contrast to this situation, the quantum efficiency of oxidation of excited rose bengal remains constant over the pH range from 5 to 12 . This $\mathrm{pH}$ difference corresponds to a change in $\triangle \mathrm{E}$ of $0.42 \cdot \alpha \mathrm{V}$ revealing that the rate of the photooxidation is independent of the free energ; of the reaction over this region of small change in $\Delta G$. This indicates that $E_{D}$ does not rest below $E_{C}$ for the dye adsorbed on the surface of the semiconductor. Thus, there appears to be no minimum rearrangement energy required of the solvent for this electron trans far to take place, and the relation of eqn. (28) does not determine the rate of this electron transfer action. It is evident that the measured activation enthalpy of $6.7 \mathrm{kcal} / \mathrm{mole}$ for this electron transfer must originate in phenomena other than the polarization of the environment involved in the rearrangement process.

The most probable source of the activation barrier can be found in the second source mentioned above: the work required for the approach and orientation of the excited dye. Attribution of the activation energy to work terms requires some exploration since the reactive species is already adsorbed on the surface of the electrode. The contribution of the free energy of adsorption to the work has already been accounted for 
in the use of the quantum efficiency of the reaction in the activation analysis. This implies that adsorption does not guarantee the orientation and approach distance that may be required for reaction.

Electron transfer from the excited dye will originate at the regions of the chromophore where the electron density of the excited electron is localized. In order for these parts of the molecule to approach the crystal lattice to within the critical distance, additional repulsion from the crystal lattice may have to be overcome. The movement of the excited species into an energetically unfavorable adsorption orientation could also be necessary. If there is an intervening solvation sheath on the surface, this too, may have to be displaced before transfer can occur. Thus, it can be seen how work terms may be necessary for activation of an adsorbed species.

It has been implicit in the discussion thus far that only the singlet state of the dye is responsible for sensitization. With the proper energetic relationship to the semiconductor, the triplet energy level of the dye should be able, in principle, to contribute to the charge injection. If the sensitization were due entirely to electrons from the triplet dye, then $\triangle E$ would represent the energy difference between the conduction band and the dye triplet energy level.

In search of the role of the triplet in sensitization, the paramagnetic ions $\mathrm{in}^{+2}$ were added to the electrolyte to serve as triplet quenchers. No change in the photocurrent was observed. It has been suggested by Tributsch ${ }^{19}$ that the lifetime of the triplet state of the adsorbed dye may already be shortened to the extent that it is relatively unaffected by the paramagnetic ions. In general, little evidence has surfaced in the literature 43 which has established the participation of the triplet state in this reaction. 


\section{Solution Photochemistry}

The photomodulated reduction observed at the cathodically polarized $\mathrm{TiO}_{2}$ was initiated by a solution species that is created by the interaction of excited rose bengal with oxygen. The role of oxygen in this reaction was demonstrated through the decrease in the cathodic current following deoxygenation by the introduction of either $\mathrm{N}_{2}$ or hydroquinone.

Investigation of the solution photochemistry of the sensitizing dyes used in this investigation has determined that their reactivity to oxygen is due almost exclusively to the activity of the triplet state. ${ }^{16}$ The reduction photocurrent would also be diminished through addition of hydroquinone because of its ability to reduce the triplet dye.

As triplets in solution, these dyes can either create singlet oxygen through energy transfer or they can be oxidized by oxygen:

$$
\begin{aligned}
& { }^{3} \mathrm{D}+\mathrm{O}_{2} \longrightarrow \mathrm{D}+\mathrm{I}_{2} \\
& { }^{3} \mathrm{D}+\mathrm{O}_{2} \longrightarrow \mathrm{D}^{+}+\mathrm{O}_{2}^{-}
\end{aligned}
$$

Both of these reactions result in products which could function as oxidants at the electrode surface:

$$
\begin{aligned}
& \mathrm{O}_{2}+\mathrm{e}^{-} \longrightarrow \mathrm{O}_{2}^{-} \\
& \mathrm{D}^{+}+\mathrm{e}^{-} \longrightarrow \mathrm{D}^{-}
\end{aligned}
$$

With the exception of tripheny 7 methane ${ }^{82}$ compounds, the dyes which sensitize the reduction at the $\mathrm{TiO}_{2}$ electrodes are known to produce singlet oxygen which can be reduced electrochemically at the $\mathrm{TiO}_{2}$ surface. Although the lifetime of singlet oxygen in aqueous solu-

tions is on the order of microseconds, 16,83 it will be created as long as the triplet dye exists in solution. The lifetimes of the triplet 
states of these dyes, however, are in the millisecond range. In the dark segment of figure $14 \mathrm{~b}$, the sensitized photoreduction requires several seconds to decay, yielding a lifetime for the reactive species of about one second. Thus, while reduction of singlet oxygen is possible it cannot account for the long lifetime of the solution species reduced at $\mathrm{TiO}_{2}$.

On the other hand, reduction of the oxidized dye in solution, eqn. (30), has been reported 82 at metal electrodes biased at potentials from $0.0 \mathrm{~V}$ to $-0.5 \mathrm{~V}$, an energy range in which the conduction band of $\mathrm{TiO}_{2}$ rests. The feasibility of such a reaction occurring at $\mathrm{TiO}_{2}$ can be estimated through a comparison of the redox potentials of the $D^{+} / D$ couples and the energy of the conduction band $E_{C}=-0.5 \mathrm{~V}$. Approximate values ${ }^{84}$ of the potentials of the $D^{+} / D$ couples for some of the dyes that exhibited this sensitized reduction current are shown in figure 52 in comparison with $E_{C}$. This comparison reveals an energy difference between the redox levels and the conduction band of 1.2 to $1.7 \mathrm{eV}$. According to theory ${ }^{85}$ the density of the unoccupied $0^{+}$ levels is described by eqn. (29) with a maximum found at a potential more negative than the $D^{+} / D$ redox level by an amount equal to the rearrangement energy, $\lambda$, for the oxidized dye. For these dyes an estimation $^{10}$ of $\lambda$ can be taken to be $0.4 \mathrm{~V}$ which indicates that there will be a sufficient density of unoccupied $D^{+}$levels at $E_{C}$ able to accept an electron from the electrode. Reduction of the oxidized dye is possible at the electrode surface in a diffusion controlled situation. It corresponds, essentially, to a back reaction of the photooxidation reaction.

There is another pathway by which this reduction of the oxidized 
dye can proceed. The superoxide radical $0_{2}^{-}$which results from reduction of oxygen at the $\mathrm{TiO}_{2}$ surface, can reduce the oxidized dye which has diffused to the electrode from the bulk of the solution. The resultant molecule may then be reduced once more at the $\mathrm{TiO}_{2}$ surface yielding a net reaction equivalent to eqn. (30):

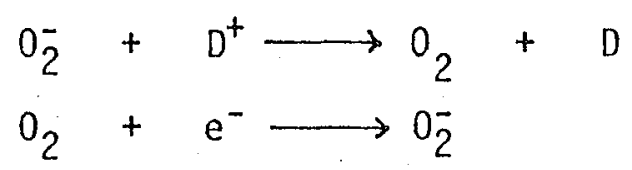

At a $\mathrm{TiO}_{2}$ electrode biased at these potentials it would not be possible to distinguish these two pathways for the reduction of the oxidized dye.

These pathways for the sensitized reduction reaction explain the regeneration of the dye on the surface which has been oxidized through the photomodulated surface reaction. Figure 49 reveals that the regeneration during a cathodic polarization sweep does not occur until $-0.25 \mathrm{~V}$, a potential at which the dark current reduction of oxygen has become discernible. Thus, the increase in the oxidative photocurrent is intimately tied to the ability of the electrode to donate an electron.

In a $D C$ measurement of the oxidative photocurrent, thie regenerative characteristics of figure 49 could not be observed. It is possible to detect it through photomodulation only because the rise times of the two currents are so different. These differing rates may be attributed to the small overlap between the conduction band density of states and the $D^{+}$energy distribution (the unoccupied levels of a $D^{+} / D$ redox couple) as compared with the Jarger overlap of the conduction band with the $D^{*}$ distribution (the occupied levels of a $D / D^{*}$ redox couple). ${ }^{63}$

Although several investigations of dye sensitization of semiconductors have utilized photomodilation to aid the detection of the resultant photocurrents, no observation of a sensitized reduction current 
has been reported. If this reduction current originated from surface states in the band gap of $\mathrm{TiO}_{2}$, the energy level of this surface state could be no more than $0.3 \mathrm{~V}$ more positive than the conduction band since the onset of the reduction current is about $0.3 \mathrm{~V}$ positive of the flatband potential. This energy difference should not preclude a contribution to the reduction current from the conduction band. Therefore, even if surface states are involved in this reaction, there is no energetic basis for the observation of this sensitized reduction reaction at $\mathrm{TiO}_{2}$ and not at $\mathrm{ZnO}$ which has a conduction band energy $\mathrm{E}_{\mathrm{C}}=-0.4 \mathrm{~V}$, very close to that for $\mathrm{TiO}_{2}$.

This directs attention to the chemical nature of the $\mathrm{TiO}_{2}$ surface which differs from $\mathrm{ZnO} . \mathrm{TiO}_{2}$ is stable under anodic polarization while $\mathrm{ZnO}$ decomposes. Reduction reactions via the $\mathrm{TiO}_{2}$ conduction band yield different Tafel slopes than the same reactions at a $\mathrm{ZnO}$ electrode. 80 Furthermore, $\mathrm{Cl}^{-}$does not appear to play a significant role in dye sensitized photooxidation at $\mathrm{TiO}_{2}$ surfaces whereas it does at $Z$ no. The sensitized reduction current may also be another of the observed differences between $\mathrm{ZnO}$ and $\mathrm{TiO}_{2}$ electrodes. 


\section{APPENDIX I}

Analys is of the data of figure $33 \mathrm{~b}$ requires expansion of eqn. (19):

$$
\begin{aligned}
\log J= & \log \left(\alpha_{1} \mathrm{e}^{-\gamma_{1} t}+\beta_{1} \mathrm{e}^{-\delta_{1} t}+\alpha_{2} \mathrm{e}^{-\gamma_{2} t}+\beta_{2} \mathrm{e}^{-\delta_{2} t}\right) \\
= & \log \left(\alpha_{1}\left(1-\gamma_{1} t\right)+\beta_{1}\left(1-\delta_{1} t\right)+\alpha_{2}\left(1-\gamma_{2} t\right)+\beta_{2}\left(1-\delta_{2} t\right)\right) \\
= & \log \left(D-\left(\alpha_{1} \gamma_{1}+\beta_{1} \delta_{1}+\alpha_{2} \gamma_{2}+\beta_{2} \delta_{2}\right) t\right) \\
& \text { where } D=\alpha_{1}+\beta_{1}+\alpha_{2}+\beta_{2} \\
= & \log D+\log (1-n t) \\
& \text { where }=\left(\alpha_{1} \gamma_{1}+\beta_{1} \delta_{1}+\alpha_{2} \gamma_{2}+\beta_{2} \delta_{2}\right) / D \\
= & \log D-n t
\end{aligned}
$$

This approximation wi11 be accurate as long as $(1 / 2) n t<.1$

where $\eta$ is the measured slope from figure 33b. For the first and largest time decay region:

$$
\begin{aligned}
& D_{1}=\alpha_{1}+\beta_{1}+\alpha_{2}+\beta_{2} \\
& \eta_{1}=\left(\gamma_{1}+\gamma_{2}+\delta_{1}+\delta_{2}\right) / D_{1}
\end{aligned}
$$

For the second time decay region:

$$
\begin{aligned}
& D_{2}=\alpha_{2}+\beta_{2}+\beta_{1} \\
& \eta_{2}=\left(\gamma_{2}+\delta_{2}+\delta_{1}\right) / D_{2}
\end{aligned}
$$

For the third decay:

$$
\begin{aligned}
& D_{3}=\beta_{2}+\beta_{1} \\
& \eta_{3}=\left(\delta_{1}+\delta_{2}\right) / D_{3}
\end{aligned}
$$

For the fourth:

$$
\begin{aligned}
& D_{4}=\beta_{1} \\
& n_{4}=\delta_{2}
\end{aligned}
$$

The data provides $\eta_{i}$ and $D_{i}$ for each decay region. Starting with $D_{4}$ and $\eta_{4}$, all of the constants in eqn. (19) can be derived. 
APPENDIX II

Conductance is defined as ${ }^{44}$ :

$$
\sigma=n e \mu
$$

where $n$ is the carrier density in $\mathrm{Cc}^{-1}$

$\mu$ is the mobility of the charge carrier

$\mathrm{e}$ is the electronic charge

For the undoped $\mathrm{ZnO}$ used in this work, $n$ was measured to be $2 \times 10^{16}$ $\mathrm{cc}^{-1}$. A value for $\mu$ may be taken as $100 \mathrm{~cm}^{2} / \mathrm{vsec}^{24}$. $\sigma$ is then $\sim .4 \mathrm{ohm}^{-1}$ $\mathrm{cm}^{-1}$. The Li doped crystal was insulating and $n$ was not determined. Both crystals were clear.

The $\mathrm{TiO}_{2}$ was found to have a carrier density of $\mathrm{n}=5 \times 10^{18} / \mathrm{cc}$. Its mobility is reported to be low, about $1 \mathrm{~cm}^{2} / V_{s e c}{ }^{80}$ The conductance is then $\sim 0.8 \mathrm{ohm}^{-1} \mathrm{~cm}^{-1}$. The crystais are sigintly grey in color. ZnS can be doped with In to yield a colorless, conducting crystal. ${ }^{86}$ Hovever, such an effort was not made with the specimens used in these studies . 


\section{REFERENCES}

1. Mees, C., James, T., eds., The Theory of the Photographic Process, (Macmillan, New York, 1966).

2. Inoue, E., Kolado, H., Yamaguchi, U., J. Phys. Chem., 67, 767 (1965).

3. Meier, H., Organic Semiconductors, (Verlag Chemie, Weinheim, 1974).

4. Dudkowski, S., Grossweiner, L., J. Opt. Soc. Am., 54, 486 (1964).

5. Heiland, G., Bauer, W., Neuhaus, M., Photochem. Photobio., 16, 315 (1972).

6. Meier, H., J. Phys. Chem., 69, 719 (1965).

7. Watanabe, T., Fujishima, A., Honda, K., Ber. Bunsen Phys. Chem., 79, $1213(1975)$.

8. Kuhn, H., Mbbius, D., Angew Chem Int. Ed., 10, 620 (1971).

9. Archer, M., J. App T. Electrochem., 5, 17 (1975).

10. Gerischer, H., Willig, F., Topics in Current Chemistry, Vol. 61, edited by A. Davison (Springer VerTag, New York, 1976), p. 31.

11. Costa, L. GiTman, P., Phot. Sci. \& Engin., 19, 207 (1975).

12. Tributsch, H., Gerischer, H., Ber. Bunsen. Phys. Chem., 73, 850 (1969).

13. Gerischer, H., Tributson, H., Ber. Bunsen. Phys. Chem., 72, 437 (1968).

14. Tritubsch, H., Gerischer, H., Ber. Bunsen. Phys. Chem., 73, 251 (1969).

15. Da1trozzo, E., Tributsch, H., Phot. Sci. \& Eng., 19, 308 (1975).

16. Gollnick, K., Advances in Photochemistry, Vol. 6, edited by W. A. Noyes (Interscience, Toronto, 1968) p. 1 .

17. Pandolph, W., Bird, G., Phot. Sci. \& Eng., 18, 340 (1974).

18. Hauffe, K., Danzmann, H., et al., J. Electrochem. Soc., 117, 993 (1970).

19. Tributsch, H., Calvin, M., Photochem. Photobio., 14, 95 (1971).

20. Memming, Tributsch, H., J. Phys. Chem., 75, 562 (1971).

21. Gutse, A., Walerys, H., Acta. Phys. Polon., 23, 581 (1963).

22. Mariano, A., Hanneman, R., J. App1. Phys., 34, 384 (1963).

23. Heiland, G., Kunstmann, P., Pfister, H., Z. Physik, 176, 485 (1963). 
24. Heiland, G., Solid State Physics, Vol. 8, edited by F. Seitz and D. Turnbul1 (Academic, New York, 1959) p. 191.

25. Harrick, N. J., Internal Reflection Spectroscopy (Interscience, New York, 1967).

26. West, W., Geddes, A., J. Phys. Chem., 68, 837 (1964).

27. Mariano, A., Hanneman, R., J. Appl. Phys., 34, 384 (1963).

28. Gerischer, H., Faraday Discussions, 58, 219 (1974).

29. Rohatgi, K., Makhapadhyay, A., Photochem. Photobio., 14, 551 (1971).

30. Poluektov, N., et a1., Z. Analit. Khim., 26, 1042 (1971).

31. Rohatgi, K., Mukhopadhyay, A., J. Phys. Chem., 76, 3970 (1972).

32. Gal, M., Kelly, G., Kurucsev, J. Chem. Soc. Faraday Trans. II, 69, 395 (1973).

33. Barrow, G., Introduction to Molecular Spectroscopy (McGraw-Hill, New York, 1962).

34. Calvert, J., Pitts, J., Photochemistry (John Wiley \& Sons, New York, 1966).

35. Schiff, L., Quantum Mechanics, 3rd ed. (McGraw-Hi11, New York, 1968).

36. Gunther, E., Moisar, E., J. Photogr. Sci., 13, 280 (1965).

37. Tani, T., Kikuchi, K., Hosoya, K., Kogyo Kagaku Lasshi, 70, 2216 (1967).

38. Tani, T., Phot. Sci. \& Eng., 17, 11 (1973).

39. Hauffe, K., Bode, U., Faraday Discussions, 58, 281 (1974).

40. McRae, E., J. Phys. Chem., 61, (562) (1957).

41. Longuet-Higgins, H., Pople, J., J. Chem. Phys., 27, 192 (1957).

42. Fujishima, A., et a1., Chem. Lett., 13 (1975).

43. Hauffe, K., Phot. Sci. \& Eng., 20, 124 (1976).

44. Kitte1, C., Introduction to Solid State Physics, 4th ed (John Wiley \& Sons, New York, 1971).

45. Ziman, J., Principles of the Theory of Solids, 2nd ed (Cambridge University Press, Cambridge, Great Britain, 1972). 
46. Pankove, J., Optical Processes in Semiconductors (Prentice-Hall, Englewood Cliffs, N.J., 1971).

47. Many, A., Goldstein, Y., Grover, N., Semiconductor Surfaces (American Elsevier, New York, 1971).

48. Gerischer, H., Photochem. Photobio., 16, 243 (1972).

49. Gerischer, H., Adv. Electro. Electrochem. Eng., Vol. 1, edited by P. Delahay and C. Tobias (John WiTey \& Sons, New York, 196) p. 139.

50. Gerischer, H., Physical Chemistry, An Advanced Treatise, VoT. IXA, edited by H. Eyring (Academic Press, New York, 1970) p. 463.

51. Myamlin, V., Pleskov., Y., Electrochemistry of Semiconductors, Plenum Press, New York, 1967).

52. Boddy, P., J. Electroana1. Chem., 10, 199 (1965).

53. Dewald, J., J. Phys. Chem. Solids, 14, 155 (1960).

54. Go mes, W., Cardon, F., Ber. Bunsen. Phys. Chem. 74, 431 (1970).

55. Lohmann, F., Z. Naturforsch., 22a, 813 (1967).

56. Hauffe, K., Range, J. . Z. Physik, Chem. NF, 64, 122 (1969).

57. Tributsch, H., Ber. Bunsen. Phys. Chem., 73, 581 (1969).

59. Fujishima, A., Hayashitani, E., Honda, K., Seisan Kenkyo, 23, 56 (1971).

59. Fujishima, A., Hayashitani, E., Honda, K., Seisan Kenkyo, 23, 31 (1971).

60. Memming, R., Photochem. Photobio., 16, 325 (1972).

61. Liptay, W., Angew. Chem. int. Edit., 8, 177 (1969).

62. Liptay, W., Ber. Bunsen. Phys. Chem., 80, 207 (1976).

63. Adelman, A., Oster, G., J. Am. Chem. Soc., 78, 3977 (1956).

64. Oster, G., Adelman, A., J. Am. Chem. Soc. 78, 913 (1956).

65. Bode, U., Hauffe, H., Ishikawa, Y., Pusch, H., Z. Physik. Chem. NF, 85, 144 (1973).

66. Gerischer, H., Mindt, W., Electrochim. Acta. 13, 1329 (1968).

67. GiTman, P., Phot. Sci \& Eng., 18, 418 (1974).

68. Marcus, R., J. Chem. Phys., 43, 2654 (1965): 
69. Fujishima, A., Honda, K., Nature, 238, 37 (1972).

70. Boddy, P., J. Electrochem. Soc., 115, 199 (1968).

71. Wrighton, M., Proc. Nat. Acad. Sci., 72, 1518 (1975).

72. Vetter, K., Schultze, J., Ber. Bunsen. Phys. Chem. 77, 945 (1973).

73. Marcus, R., Annual Review of Physical Chemistry, 15, 155 (1964).

74. Hale, J., Reactions of Molecules at Electrodes, edited by N.S. Hush, (Wiley-Interscience, New York, 1971), p. 229.

75. Dogonadze, R., Kok1. Akad. Nauk SSSR, 142, 1108 (1962); Dogonadze, R., Chizmadzhev, Y., Dok1. Akad. Nauk. SSSR, 144, 463 (1962); Dogonadze, R., Chizmadznev, Y., Dok1. Akad. Nauk. SSSR, 145, 563 (1962).

76. Cronemeyer, D., Phys. Rev., 113, 1222 (1959).

77. Fujishima, A., Sakamoto, A., Honda, K., Seisan Kenkyu, 21,450 (1969).

78. West, W., Carro17, B., Whitcomb, D., J. Phys. Chem., 56, 1054 (1952).

79. Reynolds, W., Lumry, R., Mechanism of Electron Transfer (Ronald, New York, 1966).

80. Dutoit, E., Van Meirhaege, et al., Ber. Bunsen. Phys. Chem., 79, 1206 (1975).

81. Vanden Berghe, R., Cardon, F., Gomes, W., Surf. Sci., 39, 308 (1973).

82. Memming, R., Klirsten, G., Ber. Bunsen. Phys. Chem. 76, 4 (1972).

83. Merkel, P., Kearns, D., J. Am. Chem. Soc., 94 (1972).

84. Loutfy, R., Sharp, J., Phot. Sci. Eng., 20, 165 (1976).

85. Gerischer, H., Z. Physik. Chem. NF, 26, 223 (1960); Z. Physik. Chem NF, 26, 325 (1960: Z. Physik, Chem. N. F., 27, 48 (1961).

86. T. Peterson, Lawrence Berkeley Laboratory, private communication, 1975. 
This report was done with support from the United States Energy Research and Development Administration. Any conclusions or opinions expressed in this report represent solely those of the author(s) and not necessarily those of The Regents of the University of California, the Lawrence Berkeley Laboratory or the United States Energy Research and Development Administration. 